\title{
Metal Impurity Redistribution in Crystalline Silicon for Photovoltaic Application
}

\author{
Dissertation \\ zur Erlangung des mathematisch-naturwissenschaftlichen Doktorgrades \\ "Doctor rerum naturalium" \\ der Georg-August-Universität Göttingen \\ im Promotionsprogramm ProPhys \\ der Georg-August University School of Science (GAUSS)
}

vorgelegt von

Marie Aylin Falkenberg

aus Göttingen

Göttingen, 2014 
Betreuungsausschuss

Prof. Michael Seibt, IV. Physikalisches Institut, Georg-August-Universität Göttingen

Prof. Christian Jooss, Institut für Materialphysik, Georg-August-Universität Göttingen

$\underline{\text { Mitglieder der Prüfungskommision }}$

Referent: Prof. Michael Seibt, IV. Physikalisches Institut, Georg-August-Universität Göttingen

Korreferent: Prof. Christian Jooss, Institut für Materialphysik, Georg-August-Universität Göttingen

Weitere Mitglieder der Prüfungskommission:

Prof. Hans-Ulrich Krebs, Institut für Materialphysik, Georg-August-Universität Göttingen

Prof. Vasily Moshnyaga, I. Physikalischen Institut, Georg-August-Universität Göttingen

Prof. Astrid Pundt, Institut für Materialphysik, Georg-August-Universität Göttingen

Prof. Ansgar Reiners, Institut für Astrophysik, Georg-August-Universität Göttingen

Tag der mündlichen Prüfung: 25.09.2014 


\section{Contents}

\begin{tabular}{lll}
\hline & Introduction & 1
\end{tabular}

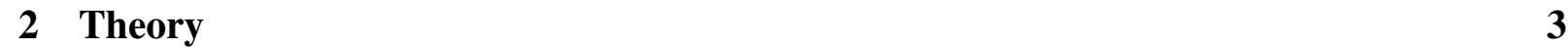

$2.1 \quad$ Iron and copper as metal impurities in silicon . . . . . . . . . . . . . . 3

2.1 .1 Solubility of iron in silicon . . . . . . . . . . . . . . . . 4

2.1 .2 Diffusion and diffusional range of iron . . . . . . . . . . . . . 5

2.1 .3 Precipitation of iron $\ldots \ldots \ldots \ldots \ldots$

2.1 .4 Precipitation of copper . . . . . . . . . . . . . . . 7

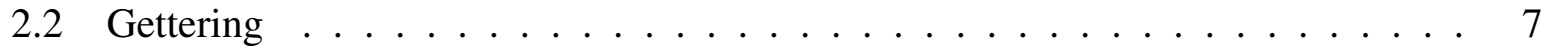

$2.2 .1 \quad$ Aluminum gettering $(\mathrm{AlG}) \ldots \ldots \ldots \ldots$. . . . . . . . . . . . . . . . . . . .

2.2 .2 Phosphorus diffusion gettering (PDG) . . . . . . . . . . . . . . . . . 9

2.3 Grain boundaries and their interaction with metal impurities . . . . . . . . . . 10

2.3.1 Grain boundaries as sinks for metal impurities . . . . . . . . . . . . . . . 11

2.3.2 Grain boundaries as sources for metal impurities . . . . . . . . . . . . . 12

2.3 .3 Memory of the sample . . . . . . . . . . . . . 13

$2.4 \quad$ Minority carrier lifetime, diffusion length and apparent concentration . . . . . . . 14

2.5 Light beam induced current (LBIC) and electron beam induced current (EBIC) . 16

2.6 Photoluminescence imaging (PL) $\ldots \ldots \ldots \ldots \ldots$

\begin{tabular}{|lll}
3 & Experimental evidence of impurity accumulation at grain boundaries & 18
\end{tabular}

3.1 Experimental methods . . . . . . . . . . . . . . . . . 18

3.2 Sample preparation . . . . . . . . . . . . . . . . . . . . . . . . . . 19

3.3 TEM lamella preparation . . . . . . . . . . . . . . . . . . . . . . . . . . . . . . . . . . . . .

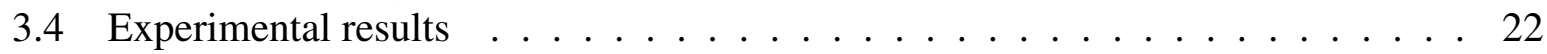

3.4 .1 Recombination-active defect at a needle-like structure . . . . . . . . . 22

3.4.2 Recombination-active defect at a honeycomb-like structure . . . . . . . . 26

3.5 Discussion of the experimental results . . . . . . . . . . . . . . 29

$3.5 .1 \quad$ Accumulation of copper at silicon nitride needles . . . . . . . . . . . . . 29

3.5.2 Accumulation of copper at silicon oxides . . . . . . . . . . . . . 29

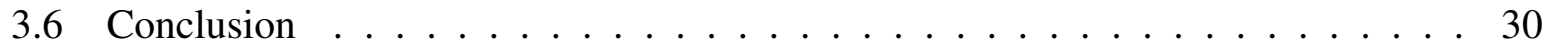

$\begin{array}{lll}4 & \text { Model description } & 31\end{array}$

4.1 Physical description of the model . . . . . . . . . . . . . . . . . 32

$4.1 .1 \quad$ Diffusion of metal impurities in the silicon bulk . . . . . . . . . . . . 32

$4.1 .2 \quad$ Aluminum gettering $(\mathrm{AlG}) \ldots \ldots \ldots \ldots \ldots$

$4.1 .3 \quad$ Precipitation in the grain $\ldots \ldots \ldots \ldots . \ldots . \ldots . \ldots . \ldots 34$

4.1 .4 Precipitation at the grain boundary . . . . . . . . . . . . . . 35

4.1 .5 Segregation at the grain boundary $\ldots \ldots \ldots \ldots$. . . . . . . . . 36

4.1 .6 Restrictions of the model . . . . . . . . . . . . . . . . . . . 37

4.1 .7 Used parameters . . . . . . . . . . . . . . . . . . 38

4.2 Technical description of the model . . . . . . . . . . . . . . . . . 40 
$4.2 .1 \quad$ PDE for interstitial metal impurities . . . . . . . . . . . . . . . 40

4.2 .2 PDE for metal impurities in the Al:Si liquid . . . . . . . . . . . . . . . . 41

$4.2 .3 \quad$ PDE for precipitation in the grains . . . . . . . . . . . . . . . . . 41

$4.2 .4 \quad$ PDE for precipitation at the grain boundary . . . . . . . . . . . . . . . . 41

$4.2 .5 \quad$ PDE for segregation at the grain boundary . . . . . . . . . . . . . . . 42

$4.2 .6 \quad$ Geometry and mesh . . . . . . . . . . . . . . . . 42

4.3 Calculation of diffusion length and apparent concentration . . . . . . . . . . . 44

5 Simulation studies on impurity accumulation at grain boundaries 46

5.1 Comparison of phosphorus diffusion gettering and aluminum gettering in a one dimensional Model . . . . . . . . . . . . . 46

5.2 Aluminum gettering with grid fingers or a grain boundary in a two dimensional

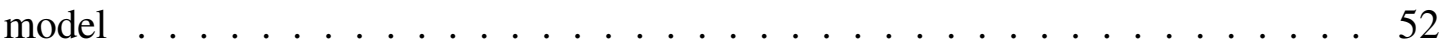

5.2 .1 Homogeneous aluminum gettering for comparison . . . . . . . . . 54

5.2 .2 Aluminum gettering with grid fingers $\ldots \ldots \ldots \ldots$. . . . . . . . . . . . . . . . . 56

$5.2 .3 \quad$ Aluminum gettering in presence of a grain boundary $\ldots \ldots$. . . . . . 58

5.2.4 Comparison of the integral concentrations for the different gettering sce-

narios ............................. 60

5.3 Impurity accumulation at grain boundaries . . . . . . . . . . . . . . . 62

$\begin{array}{lll}5.3 .1 & \text { Simulations of as-grown wafers for comparison with LBIC measurements } 62\end{array}$

5.3.2 Variation of temperature and cooling rate for comparison with LBIC measurements ..................... 68

$5.3 .3 \quad$ Simulations for comparison with PL measurements . . . . . . . . . . . 75

5.3.4 Simulations of precipitation and dissolution of precipitates for comparison with PL measurements . . . . . . . . . . . . . . . . . 80

5.3.5 Conclusion from the comparison of LBIC and PL measurements . . . . . 86

5.4 Investigation of the influence of the parameters on the example of the as-grown

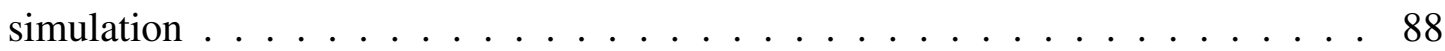

$5.4 .1 \quad$ Variation of grain size $\ldots \ldots \ldots \ldots$. . . . . . . . . . 88

5.4.2 Variation of total impurity concentration . . . . . . . . . . . . . . . 91

$5.4 .3 \quad$ Variation of precipitate density at the grain boundary . . . . . . . . . . . 94

5.4 .4 Variation of precipitate density in the grain $\ldots \ldots . . \ldots 95$

5.5 Segregation versus precipitation $\ldots \ldots \ldots \ldots$. . . . . . . . . 97

\begin{tabular}{lll}
\hline 6 & Summary and outlook & 107
\end{tabular}

\begin{tabular}{lll}
\hline 7 & Appendix & 111
\end{tabular}

7.1 Mesh settings and boundary conditions for iron in-diffusion . . . . . . . . . . 111

7.2 Mesh settings at the grain boundary $\ldots \ldots \ldots \ldots$. . . . . . . . . . . . . . . . . . .

\begin{tabular}{llr}
\hline 8 & Acknowledgement & 122
\end{tabular} 


\section{Introduction}

Photovoltaics, like all renewable energies, is an alternative way to obtain electricity in order to preserve our planet for us and our descendants. Renewable energies are a great opportunity for us to meet the growing demand for electricity in a sustainable, pollution-free, save and recyclable way. The advantages are striking, regarding fossil fuels becoming scant, emission of $\mathrm{CO}_{2}$ polluting our atmosphere, the risk of nuclear disasters and nuclear waste disposal problems.

Photovoltaics is the technology to directly convert the radiation energy from the sun into electrical energy. It has come a long way from the first intentionally built PV device in 1883 [1], to solar cell mass production and a worldwide electricity capacity of over 100 gigawatt (GW) in 2012 [2]. However, to make photovoltaics profitable and competitive today, further fundamental research is needed to optimize the cost per power. In the year 2013, $90 \%$ of the produced photovoltaic modules were made out of silicon [3], which is one of the most abundant elements in the earth's crust and is ecologically harmless. Out of the silicon material $\frac{3}{4}$ was multicrystalline silicon due to its lower costs and energy expenses during production. The disadvantages of multicrystalline silicon in comparison to monocrystalline silicon are the presence of extended defects like grain boundaries and dislocations and a high amount of metal impurities potentially reducing the solar cell efficiency.

In a solar cell, electrical power is generated from a semiconductor material when it is illuminated with light (inner photo effect). The energy of the light is absorbed and generates excess charge carriers which lead to a voltage.

The maximum theoretical efficiency for a solar cell as calculated by William Shockley and Hans Queisser (Shockley-Queisser limit) is not reached in real solar cells due to recombination losses. The most important recombination process in silicon solar cells is the Shockley-ReadHall recombination due to metal impurities and extended defects. Both increase the recombination probability for charge carriers, leaving less excess carriers to contribute to the electrical current and therefore reducing the efficiency [1, 4, 5, 6, 7, 8].

Most frequent impurities are the 3d-transition metals like iron, copper and nickel [9, 10, 11], which are unintentionally introduced into the silicon material during crystal growth and cell production. Especially the fast diffusing metals like iron and copper are detrimental because they are unavoidable and have a high mobility even at low temperatures.

In order to reduce the detrimental effect of metal impurities on the efficiency, they can be spatially redistributed by including gettering processes in solar cell processing schemes [12, 8 , 13, 14]. During gettering the impurities move to electrical inactive areas of the solar cell like the highly phosphorus doped emitter (phosphorus diffusion gettering) or the aluminum backside contact (aluminum gettering) if a standard p-type Si solar cell is considered.

Several authors propose another technique to reduce the detrimental effect of metal impurities: the intentional redistribution of impurities at few large accumulation sites, e.g. at extended defects, to increase the distance of recombination active defects and improve the lifetime of the charge carriers in the defect free regions [15, 16, 17]. 
In general, extended defects can act as sinks for impurity atoms. This accumulation process is reversible, thus extended defects which are decorated with impurities can act as sources for new impurity atoms during temperature treatments and/ or gettering [5, 10, 15]. While under certain temperature treatments they can reduce the impurity concentration within the grains, the defects can recontaminate the grains under other conditions and reduce the efficiency and the success of external gettering processes. Fundamental research on atomistic scale [18, 19, 20, 21, 22] and on grain-size scale $(1-10 \mathrm{~mm})[23,24,25,26,27]$ is needed to understand the interaction of impurities with extended crystallographic defects and the influence on gettering processes to optimize defect engineering and gettering for multicrystalline material. Besides experimental measuring methods (like LBIC, EBIC, PL, NAA, DLTS and TEM ${ }^{1}$ ), simulations are a powerful tool on the way of understanding and interpreting the underlying physics. While there are many simulations modeling gettering of impurities [14, 28, 29, 30, 31, 32, 33, 34], there are only a few authors investigating the accumulation of impurities at grain boundaries by simulations [25, 35].

This work contains both, experimental investigations of impurity accumulation at extended defects on the atomistic scale and simulations on the wafer scale of the redistribution of impurity concentration during temperature treatments and gettering processes in presence of grain boundaries.

The following sections give an introduction on metal impurities in silicon with the focus on iron in section 2.1. on gettering of metal impurities in section 2.2 and on the mechanisms of impurity accumulation at grain boundaries in section 2.3 . In section 2.4 the correlation between metal impurities and diffusion length of the excess carriers is described. This correlation is important to connect the simulations with experimental results, e.g. with LBIC measurements. The methods of LBIC/ EBIC and PL are described in section 2.5 and 2.6 as the technique of EBIC is used in this work, and LBIC and PL measurements are used to compare with simulations.

The result section consists of two parts. Subject of the first part is the detailed investigation of accumulation of impurities at extended defects: section 3 describes an experimental method, combining EBIC, FIB and TEM techniques, which was developed to investigate the distribution, the atomic structure and the chemical nature of selected extended recombination active defects at high resolution, but low necessary defect density. Results obtained with this method are presented which give experimental evidence of precipitation of metal impurities at grain boundaries causing extended recombination active defects.

In the second part, simulations of the metal impurity distribution on the example of iron on wafer scale are presented. The focus lies on the distribution of the iron concentration after different temperature treatments and gettering processes in the presence of a grain boundary and other precipitation sites. The physical model used for the simulations and its implementation are described in section 4. Section 5.1 shows a simulation study comparing the efficiency and kinetics of two different gettering techniques - aluminum gettering and phosphorus diffusion gettering -

\footnotetext{
${ }^{1}$ The abbreviations stand for: Light Beam Induced Current (LBIC), Electron Beam Induced Current (EBIC), PhotoLuminescence Imaging (PL), Neutron Activation Analysis (NAA), Deep Level Transient Spectroscopy (DLTS) and Transmission Electron Microscope (TEM)
} 
in the presence of precipitates, while section 5.2 focuses on the effect of a grain boundary on the efficiency and kinetics during aluminum gettering. In section 5.3 the redistribution of impurities during temperature treatments without gettering are presented and the modeled concentration profiles across the grain boundary are compared with LBIC and PL measurements to investigate the interaction of impurities with a grain boundary during different temperature treatments. Additionally several parameters variations are presented to give an insight into the influence of the different parameters on the resulting concentration profiles. The simulations of the former chapters assume only precipitation as mechanisms of impurity accumulation at a grain boundary, which is consistent with the experimental results in section 3 and will be shown to be sufficient to reproduce the experimental LBIC and PL measurements. In section 5.5 the influence of segregation in comparison to precipitation as mechanism of impurity accumulation at a grain boundary is investigated and will be shown to be negligible in most cases, verifying the approach of using only a precipitation model for the impurity accumulation at a grain boundary in the former sections.

\section{Theory}

\subsection{Iron and copper as metal impurities in silicon}

Transition metals like iron, copper, nickel and gold have a detrimental effect on the efficiency of solar cells because transition metals, their complexes and precipitates introduce deep energy states into the band gap. The deep energy states act as recombination centers for the charge carriers and therefore reduce the lifetime of the excess carriers produced by light absorption.

The abundance of iron and copper and their fast distribution due to their high diffusivity make iron and copper contamination during solar cell production unavoidable [7, 19]. Additionally, iron and copper are highly reactive. For example, more than 30 complexes of iron are reported with about 20 energy levels in the silicon band gap [36]. Sources for contamination are, for example, the polysilicon feedstock, the crucible during crystal growth and the equipment during processing [5, 7, 37].

Figure 1, taken from Buonassisi et al. [10], shows neutron activation analysis data of total metal content in ingot-grown, ribbon, and sheet mc-Si materials illustrating that iron and copper are among the most abundant metal impurities with typical concentrations of $10^{13}-10^{15} \mathrm{~cm}^{-3}$ [17], which corresponds to $2 \cdot 10^{-4}-2 \cdot 10^{-2}$ ppma (parts per million atoms).

The rather high concentrations typically observed stand in contrast with the tolerable amount of iron in solar cells. Reiss et al. [38] show that in CZ solar cells an iron concentration of $5 \cdot 10^{11} \mathrm{~cm}^{-3}$ can already result in an efficiency loss of 3-4 percent. Consistent with these observations, the iron concentration has to be below approximately $5 \cdot 10^{11} \mathrm{~cm}^{-3}$ for a wafer thickness of $180 \mu \mathrm{m}$ according to the conversion from diffusion length to interstitial iron concentration by Istratov et al. [7]. This results from the simple approximation that the diffusion length of the excess carriers in a solar cell should be larger than the wafer thickness in order for the carriers to reach the contacts before they recombine. Measuring techniques for iron and copper in silicon are, for example, DLTS [39], PL [40], NAA [41], LBIC [42], EBIC and TEM [43]. A com- 


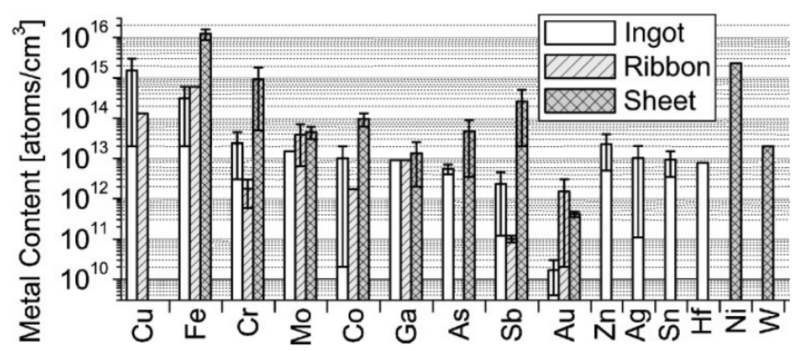

Figure 1: Neutron activation analysis data of total metal content in ingot-grown, ribbon, and sheet mc-Si materials from Buonassisi et al. [10].

bination of several methods has to be used to gain information of the amount, the location and the chemical state of the iron as described in more detail in section 3.1. A short description of EBIC/LBIC and PL will be given in sections 2.5 and 2.6, respectively.

Iron and copper dissolve and diffuse interstitially in silicon [36, 44]. At room temperature copper is predominantly positively charged [45] in p-and n-type silicon, while interstitial iron is dominantly neutral in n-type silicon and dominantly positive in p-type silicon [36]. The positively charged iron and copper impurities pair with shallow acceptors due to electrostatic attraction.

Important for this work is the positively charged iron which pairs with negatively charged boron atoms. The FeB pairs can be separated by temperature above approximately $200^{\circ} \mathrm{C}$ [7] or by illumination. At higher temperatures (above $830^{\circ} \mathrm{C}$ ) the dominant species is neutral iron in both $\mathrm{n}$ - and p-type silicon [36].

\subsubsection{Solubility of iron in silicon}

The solubility of transition metal impurities in silicon is determined by the equilibrium of the solid solution with the silicon-richest phase of the binary Si:M phase diagram at a given temperature. For iron and most other transition metals, the silicon-richest phase below the eutectic temperature ( $1207^{\circ} \mathrm{C}$ for iron) is the metal disilicide $M S i_{2}$ [16].

Below the eutectic temperature, the solubility in intrinsic silicon can be calculated by the following Arrhenius law:

$$
C_{e q}=C_{L} \cdot \exp \left(\frac{\Delta S \cdot T-\Delta H}{k T}\right)
$$

with $C_{L}$ being the density of interstitial sites which can be occupied by the metal.

$\Delta G=\Delta S \cdot T-\Delta H$ is the excess Gibbs free energy of the metal impurity with the solution entropy $\Delta S$ and the solution enthalpy $\Delta H$. In this description only the neutral impurity and the dominant interstitial species is considered.

Table 1 lists values for $C_{L}, \Delta S, \Delta H$ determined with NAA by Weber [46] which were used to plot the temperature dependent solubility for iron in silicon in figure 2 in in section 2.1.2. The figure shows the strong decrease of the solubility with decreasing temperature. 


\begin{tabular}{lll}
\hline \hline Parameter & Value & Description \\
\hline$C_{L}$ & $5 \cdot 10^{22} \mathrm{~cm}^{-3}$ & Density of interstitial sites in silicon \\
$\Delta S$ & $8.2 \cdot k$ & Solution entropy of Fe in precipitates $/ \mathrm{Si}$ \\
$\Delta H$ & $2.94 \mathrm{eV}$ & Solution Enthalpy of Fe in precipitates $/ \mathrm{Si}$ \\
$D_{0}$ & $9.5 \cdot 10^{-4} \mathrm{~cm}^{2} / \mathrm{s}$ & Constant for diffusion coefficient $\mathrm{D}$ \\
$\Delta H^{m}$ & $0.65 \mathrm{eV}$ & Activation energy of interstitial Fe in Si
\end{tabular}

Table 1: List of used values for the diffusion coefficient from DLTS measurements by Isobe et al. [47] and for the solubility from NAA measurements by Weber [46].

For low temperatures between $600-800^{\circ} \mathrm{C}$ Murphy and Falster [48] showed that for the calculation of the solubility different values have to be used than for higher temperatures. Therefore the values from Murphy and Falster [48] were used for temperatures below $800^{\circ} \mathrm{C}$.

\subsubsection{Diffusion and diffusional range of iron}

In silicon iron diffuses via an interstitial mechanism [36], which is a thermally activated jump movement [49]. It is described by the temperature dependent diffusion coefficient D:

$$
D=D_{0} \cdot \exp \left(-\frac{\Delta H^{m}}{k T}\right)
$$

with the temperature $T$, the migration barrier for an atom to jump from one stable position in the crystal to the next $\Delta H^{m}$, and the Boltzmann constant $k$. The prefactor $D_{0}$ depends on the species of diffusing atoms and on the host lattice. The prefactor $D_{0}$ and the migration barrier $\Delta H^{m}$ have been determined experimentally by several authors (please see Istratov et al. [36] and references therein) and the data is in close accordance. While it is not clear to which amount this diffusion coefficient is dominated by the neutral and the positive species of iron, these experimentally determined values are sufficient to quantitatively model the diffusion of iron in silicon. In this work the data from Isobe et al. [47], derived from DLTS measurements, is used. The values for $D_{0}$ and $\Delta H^{m}$ are given in table 1 in section 2.1.1.

Figure 2 shows the diffusion coefficient and the solubility for iron in silicon according to the data from table 1. It illustrates that the solubility decreases strongly with decreasing temperature, while the diffusion coefficient decreases only slightly. This results in unusual high mobilities at high supersaturation levels during cooling to room temperature, causing a high driving force for precipitation and a strong influence of the cooling rate on the final interstitial impurity concentration.

For a given temperature $T$ and cooling rate $\beta$ an estimation for the diffusional range $R_{M}$, meaning the mean distance an impurity atom travels during cooling from its initial position in a sample, can be calculated according to Schröter et al. [50]:

$$
R_{M}=\sqrt{6 \cdot D_{0} \cdot \beta \int_{T_{0}}^{T_{m}} \exp \left(-\frac{\Delta H^{m}}{k \cdot T}\right) d T}
$$


where $T_{0}$ is the temperature to which the sample is cooled and $T_{m}$ is the temperature at which supersaturation of the metal impurity occurs in the sample.

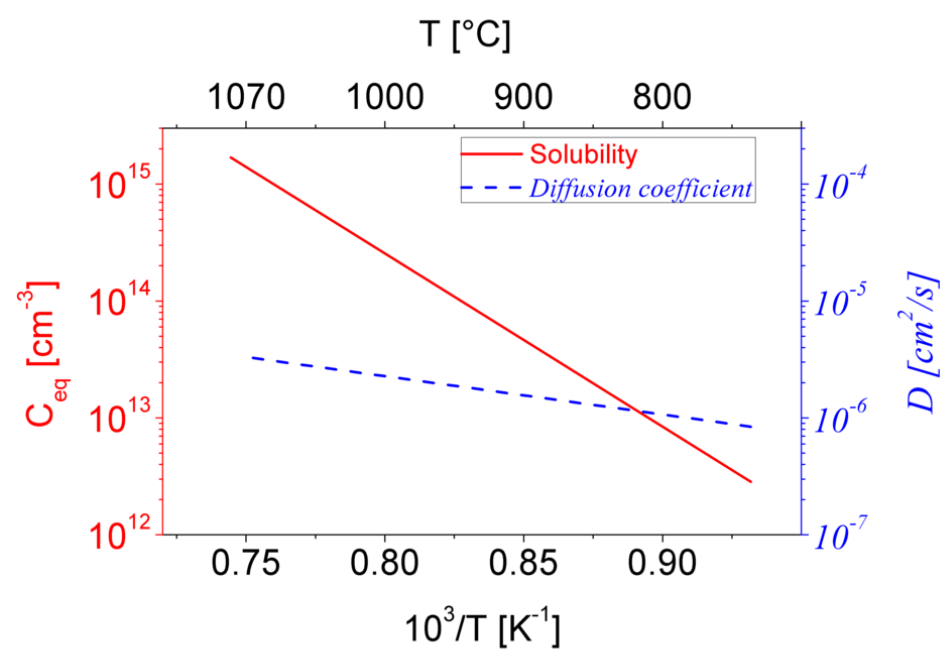

Figure 2: Diffusion coefficient $D$ and solubility $C_{e q}$ of iron in silicon versus temperature. The values for the calculation of $D$ and $C_{e q}$ are listed in table 1 and are taken from Schröter and Seibt [51]. With decreasing temperature, the solubility decreases rapidly, while the diffusion coefficient decreases only slowly. Therefore, the iron impurties are still mobile at high supersaturation levels, which results in high driving force for precipitation and a strong dependence of precipitation on the cooling rate.

\subsubsection{Precipitation of iron}

Precipitation can occur when the impurity concentration exceeds the solubility. Iron precipitates heterogeneously at dislocations, grain boundaries and precipitates [52, 16]. The precipitation of iron at extended defects (grain boundaries and dislocations) will be described in section 2.3 . Several iron silicide precipitates like $\mathrm{FeSi}_{2}, \mathrm{FeS} i$ and oxygen containing precipitates [7] have been observed. The lattice parameter for iron precipitates varies only slightly from the lattice parameter of the silicon matrix. The role of silicon vacancies and interstitials in the precipitation process is negligible. The low volume misfit results in low strain fields for iron precipitates.

In as-cut wafers a significant amount of the iron concentration is in form of precipitates [15, 9] due to the slow cooling after crystallization. Liu and Macdonald [53] report that the strength of precipitation depends on the temperature, the supersaturation ratio and the density and type of precipitates.

While interstitial iron is observed to be more detrimental on the lifetime of the minority carriers, the immobility of precipitated iron is a major drawback during gettering processes [54, 55]. In order to remove precipitated iron by gettering, the precipitates have to be dissolved first. 
The influence of precipitates and a contaminated grain boundary on the gettering kinetic will be shown in section 5.1 and in section 5.2 respectively.

\subsubsection{Precipitation of copper}

Copper precipitates homogeneous as plate-shaped precipitates and heterogeneous at grain boundaries and dislocations as $\mathrm{Cu}_{3} \mathrm{Si}$ [19]. Seibt et al. [44] showed that the homogeneous precipitation merges into heterogeneous colony growth at dislocations because the plate-shaped precipitates are metastable and decompose via silicon self-interstitial emission into spherical precipitates due to strain relaxation. For copper the volume expansion is estimated to 150 percent by Schröter et al. [50]. The result are colonies of copper silicide precipitates with a planar arrangement. Due to the even higher mobility of copper compared to iron, almost all copper is found in precipitates after quenching, often with an inhomogeneous distribution of precipitates at grain boundaries and dislocations [10].

\subsection{Gettering}

Since transition metals and especially iron are not avoidable during crystal growth and solar cell processing, mechanisms to reduce the impurity concentrations are needed. A common method in solar cell production is the redistribution of impurities into electrical inactive regions of the device. This redistribution is called gettering. Another technique is to redistribute the impurities at a few accumulation sites to reduce the diffusion length in between these accumulation sites. The latter method will be described in more detail in section 2.3 .

During gettering, impurities are dissolved, diffuse to a gettering region and are captured at the gettering site. There are different gettering mechanisms namely relaxation gettering, segregation gettering and injection-gettering [56]. During relaxation gettering, the impurities precipitate in the gettering region due to a supersaturation in the bulk material. An example is gettering by silicon oxide precipitates (internal gettering) in microelectronic devices. Injection gettering is caused by the injection of intrinsic point defects due to diffusion processes or surface reactions. For segregation gettering, a discontinuity of solubility between the bulk material and the gettering region is used. The driving force is the lower chemical potential of the impurity in regions with higher solubility. The discontinuity can be between phases (e.g. between the liquid and the solid silicon during crystal growth), different materials (aluminum gettering), regions of different doping levels (phosphorus diffusion gettering), or between regions of different strain, e.g. in the vicinity of an extended defect. The advantage of segregation gettering in comparison to relaxation gettering is that no supersaturation is needed. The two gettering techniques discussed in this work are aluminum gettering and phosphorus diffusion gettering.

\subsubsection{Aluminum gettering (AlG)}

The underlying physical mechanism of aluminum gettering is segregation [57, 58, 33]. It is a very successful process and can easily be included into the solar cell production process because 
an aluminum layer can be used to form a backside contact and a backside field (BSF). The thickness of the aluminum layer for AlG is usually in the range of $2-10 \mu \mathrm{m}$. The gettering effect of aluminum gettering has been investigated by many authors [13, 8, 59, 60, 55]. At elevated temperatures above the eutectic temperature $\left(577^{\circ} \mathrm{C}\right)$ of the binary phase diagram an $\mathrm{Al}$ :Si liquid is formed with the silicon [16]. The formation happens fast, usually even during heating up to the annealing temperature. Due to the fast establishment of the gettering layer, the efficiency of $\mathrm{AlG}$ is only limited by thermodynamics, as will be shown in this work in section 5.1. This is an advantage compared to other gettering techniques for which the gettering layer is established by in-diffusion as it is the case for e.g. phosphorus diffusion gettering (PDG).

The solubility of metal impurities in the $\mathrm{Al}$ :Si liquid is at temperatures of $900-1100{ }^{\circ} \mathrm{C}$ by 4-5 orders of magnitude higher than the solubility in silicon [39]. This causes the segregation effect between the silicon and the Al:Si liquid and can be described comparable simple (please see section 4.1.2) in terms of the segregation coefficient $S_{A l}$, which is defined as the ratio of the solubility in the Al:Si liquid $C_{A l}^{e q}$ and the solubility in the silicon bulk $C_{S i}^{e q}$ :

$$
S_{A l}=\frac{C_{A l}^{e q}}{C_{S i}^{e q}}
$$

It has to be kept in mind that the segregation coefficient decreases with increasing temperature. However, the segregation effect during aluminum gettering is active even at elevated temperatures [39], which makes it possible to remove precipitated metal impurities of high concentration [61]. Its temperature dependence for iron in silicon has been measured by Abdelbarey et al. [39]. As shown in figure 3 taken from Abdelbarey et al. [39], the measured segregation $S_{A l}^{*}$ coefficient deviates from the segregation coefficient $S_{A l}$ estimated from the ratio of the metal solubilities according to the binary Al:M and Si:M phase diagram. The authors explain this discrepancy with an increased solubility of iron in silicon in the equilibrium with the ternary Al-doped $\alpha-F e S i_{2}: A l$ compared to the solubility with respect to the binary $\alpha-F e S i_{2}$.

For the present simulations, the experimentally determined segregation coefficient $S_{A l}^{*}$ is used. In this work mainly AlG is used to investigate the influence of grain boundaries on gettering processes. The comparable simple underlying physics makes AlG a good tool in experiments and simulations for fundamental research, even though its potential to getter metal impurities can not fully be accessed in practical solar cell production [55], because the typical applied firing process is too short to allow a significant amount of impurities to move to the gettering layer. 


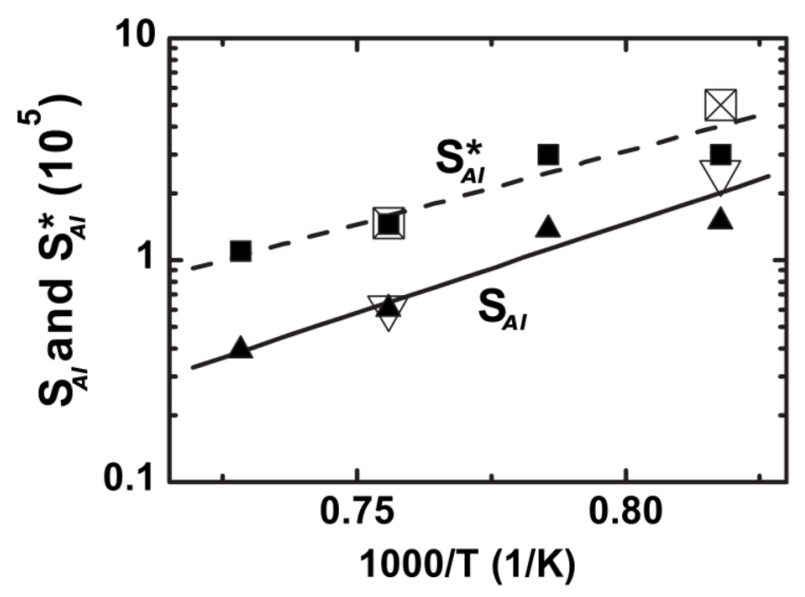

Figure 3: Arrhenius plots of the segregation coefficient $S_{A l}$ determined from the ratio of the metal solubilities according to the binary $\mathrm{Al}: \mathrm{M}$ and $\mathrm{Si}: \mathrm{M}$ phase diagram and of the experimentally determined segregation coefficient $S_{A l}^{*}$. Symbols show data for one-step (open symbols) and two-step (closed symbols) AlG. Dashed and solid lines are least-square fits. The figure is taken from Abdelbarey et al. [39].

\subsubsection{Phosphorus diffusion gettering (PDG)}

One of the main gettering techniques used in solar cell production is phosphorus diffusion gettering because phosphorus diffusion is used for emitter formation in p-type solar cells and for back surface field formation (BSF) in n-type solar cells. The reduction of interstitial iron concentration due to phosphorus diffusion gettering has been investigated by many authors [62, 63, 64, 55, 5].

During phosphorus diffusion, a complex interplay of many mechanisms takes place, the most important mechanisms being segregation due to the Fermi level effect and pairing, and injection gettering due to the injection of silicon self-interstitials during the dissociation of phosphorusinterstitial pairs (PI). As stated in Kveder et al. [30] the diffusion of phosphorus can well be described via phosphorus-interstitial (PI) pairs and via phosphorus-vacancy (PV) complexes. The two diffusion mechanisms compete with each other, the kick-out mechanism for the PI pairs being dominant at low phosphorus concentrations and the diffusion via the phosphorus-vacancy complex being dominant at high phosphorus concentrations, i.e. the surface region of the wafer. Injection gettering is only active for substitutional metal impurities, while it plays no role for the interstitially diffusing $3 \mathrm{~d}$ transition metal impurities, for which the supersaturation of silicon self-interstitials has no effect. At high phosphorus concentrations, which exceed the solubility of phosphorus in silicon, a gettering effect due to silicide precipitation has been observed. This is described in more detail in Schröter et al. [50] and in Seibt and Kveder [16]. 


\subsection{Grain boundaries and their interaction with metal impurities}

Different types of grain boundaries are found in multicrystalline silicon, mainly $\Sigma 3$ twin boundaries, higher order twin boundaries and random boundaries including small angle grain boundaries [16, 65]. It has been observed that grain boundaries show a recombination activity, i.e. they serve as recombination centers for the excess minority carriers in a solar cell [66, 67]. The recombination activity of grain boundaries depends not only on the grain boundary type [67, 68], but also on the contamination level of metal impurities [66, 67]. The latter indicates an interaction of grain boundaries with metal impurities. However, the interplay of the recombination at the grain boundary and the electronic properties of the impurity accumulation at the grain boundary, which results in the electrical behavior of the grain boundary, is not well understood.

The two most discussed mechanisms for the accumulation of impurities at grain boundaries are precipitation and segregation [16, 69, 52, 70]. Precipitation at nucleation sites in grain boundaries occur due to a supersaturation in the bulk material. The decreased impurity concentration near the grain boundary cause a concentration gradient which is the driving force for impurity diffusion towards the grain boundaries. Segregation results from a discontinuity of solubility between the bulk material and the grain boundary. It is described in terms of a segregation coefficient $S_{g b}$ giving the ratio between the solubility in the grain boundary and the silicon matrix. The driving force results from a lower chemical potential in regions with higher solubility. Precipitation seems to be the dominating effect for impurity accumulation because there is only little experimental evidence for segregation as mechanism of impurity accumulation at grain boundaries [16, 69], while there is solid evidence for precipitation at grain boundaries as shown in this work (please see section 3, Falkenberg et al. [43] and Falkenberg and Seibt [71] and by several other authors [72, 73, 74]. Consistently, simulations in this work also indicate that segregation has only minor effect and experimental results can well be modeled by using a model with only precipitation as mechanism of impurity accumulation (please see section 5.5 .

As precipitated iron is less detrimental for the solar cell efficiency than the interstitial component, several authors [17, 15, 75, 14, 26] propose 'internal gettering', i.e. temperature treatments to intentionally redistribute iron (and other metal impurities) at a few accumulation sites in order to reduce concentration in the grains. In this work, this treatments will not be called 'internal gettering' because the term is already used for gettering by silicon oxide precipitates in microelectronic devices. The optimal temperature for the intentional redistribution of impurities at precipitation sites is, when the solubility is low while the diffusion coefficient $D$ is still large. For typical conditions in a multicrystalline silicon sample, optimum temperatures of $500^{\circ} \mathrm{C}-600^{\circ} \mathrm{C}$ are reported [76, 77, 75, 15].

The drawback of this method is that accumulation at grain boundaries and other accumulation sites is reversible, i.e. subsequent high temperature treatments can cause re-contamination of the grains, when grain boundaries and other accumulation sites serve as source for impurities.

In the following text a short review of the experimental observations about grain boundaries as sinks an sources for impurities will be given. 


\subsubsection{Grain boundaries as sinks for metal impurities}

Figure 4] shows an LBIC map, measured by Krenckel [42], from an as-grown sample which indicates that grain boundaries served as sinks for interstitial iron during the crystallization cooling process.

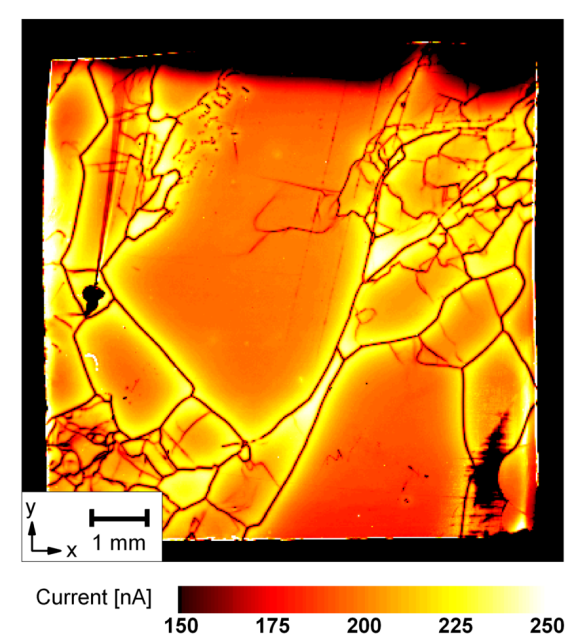

Figure 4: LBIC map from measurements by Krenckel [42]. Low currents (dark) indicate low diffusion length which are interpreted as high interstitial iron concentration, high currents (bright) indicate high diffusion length which are interpreted as low interstitial iron concentration. Grain boundaries can be seen as dark lines surrounded by bright regions of low interstitial iron concentration called 'denuded zone'.

As will be described in the following section 2.4, low currents can be interpreted as high interstitial iron concentration and high currents as low interstitial iron concentration, when assuming that the diffusion length is limited by the interstitial iron concentration.

The figure shows regions of high currents, i.e. of low interstitial iron concentrations in the vicinity of the grain boundaries, which will be called denuded zones in the further text. These denuded zones in the vicinity of grain boundaries and regions of low interstitial iron concentration at high dislocation densities in as-grown wafers have also been observed by many other authors $[24,40,10,27,25,68,78]$ and are the result of impurity accumulation during the slow cooling process after crystallization. During cooling the temperature dependent solubility $C_{e q}$ of the metal impurity in silicon decreases. At the interstitial equilibrium temperature $T_{e q}^{i}$, at which the solubility equals the interstitial impurity concentration in the sample, precipitates may nucleate at the grain boundaries and other nucleation sites within the grains. In the further cooling process the precipitates grow as long as the diffusion coefficient $D$ is large enough for the impurities to reach the precipitates. The cooling rate has a strong influence on the width of the denuded zones. This is also true for all subsequent temperature treatments after crystallization [42, 35].

The figure also shows that not all grain boundaries show the same denuded zone, i.e. the 
process of accumulation differs for different grain boundaries. One possible reason for this is the observation that different types of grain boundaries show different strength of impurity accumulation. A trend of higher decoration of small angle grain boundaries is observed [10, 67].

\subsubsection{Grain boundaries as sources for metal impurities}

As the precipitation at grain boundaries and other precipitation sites is reversible, (subsequent) annealing with an annealing temperature above the interstitial equilibrium temperature $T_{e q}^{i}$, at which the solubility exceeds the interstitial iron concentration in the sample, causes (partial) dissolution of the precipitates. During this process, grain boundaries serve as sources for interstitial metal impurities and re-contaminate the grains. A typical observation is an enriched region in the vicinity of grain boundaries. This will be illustrated by the simulations in this work in section 5.3.1.

The behavior of grain boundaries as sources can be investigated experimentally for short annealing times with subsequent fast cooling. For sufficiently high cooling rates, there is not enough time for precipitation and the state of the sample after the annealing is 'frozen'. The annealing step has to be sufficiently short to avoid that an equilibrium state is established, the diffusion process homogenizes the interstitial iron concentration [17] and the enriched zones disappear.

Also a higher interstitial iron concentration in the vicinity of grain boundaries and regions of high dislocation densities after gettering has been observed by several authors [24, 5, 25, 79]. Figure 5 shows an example of enriched zones in the vicinity of grain boundaries after an extended gettering treatment. Shown are interstitial iron concentration maps calculated from PL measurements of two neighbor wafers with similar grain structure. The figures are taken from Liu et al. [24]. A comparison of the as-cut wafer with a neighbor wafer, which was treated with an extended gettering process, shows that the same grain boundaries have denuded zones of interstitial iron in the as-cut wafer while they have enriched zones after the gettering treatment.

The dissolution of precipitates during gettering is caused by an increase of the interstitial iron concentration below the solubility. The experimental observations of re-contamination during gettering and other temperature treatments shows that the interaction of impurities with grain boundaries plays an important role for defect engineering and an understanding of the underlying physics is necessary to predict the behavior during temperature treatments. Sections 5.1 and 5.2 will show that the dissolution of precipitates in the grains and at grain boundaries not only causes re-contamination of the grains, but possibly also influences the gettering kinetics. 


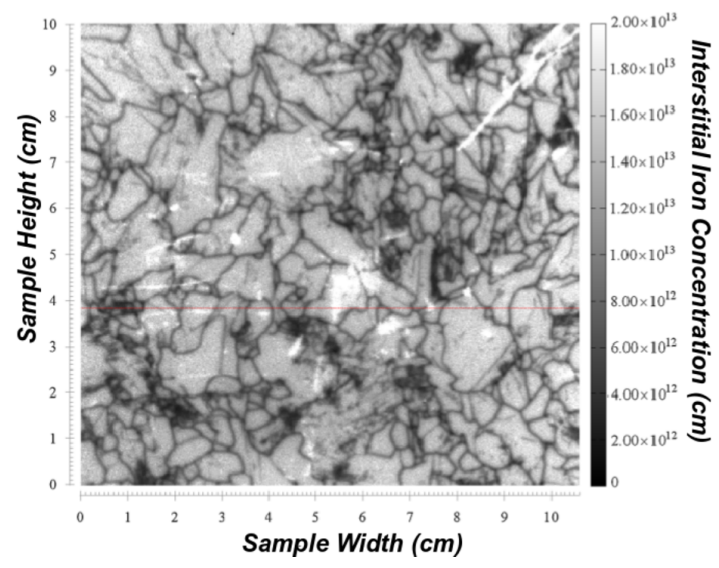

(a) Wafer in the as-cut state: grain boundaries as sinks

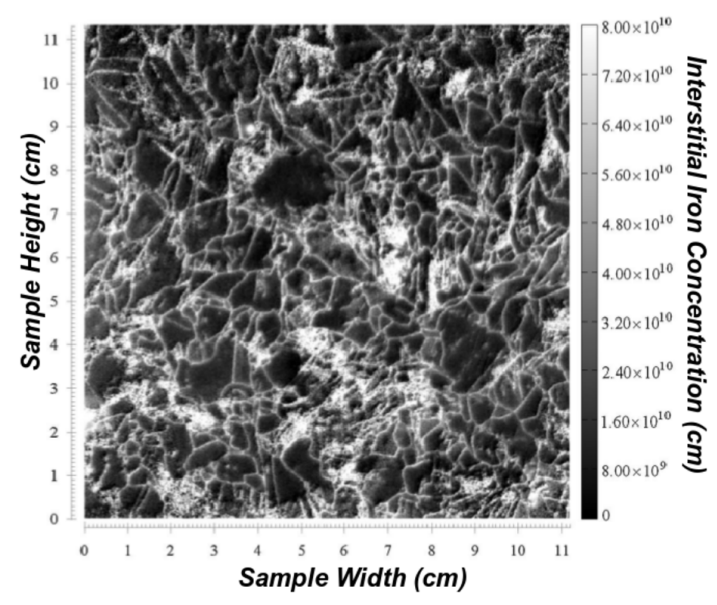

(b) Wafer after extended gettering treatment: grain boundaries as sources

Figure 5: PL maps taken from Liu et al. [24] showing the interstitial iron concentration of two neighbor wafers in the as-cut state $5(5(\mathrm{a})]$ and with a subsequent extended gettering treatment (5(b)). The same grain structure is observed, however with different iron concentrations in the vicinity of the grain boundaries: the denuded zones in the as-cut wafer appear as zones of enriched iron concentration in the wafer treated with an extended gettering process. The red line in figure 5(a) indicates a line scan not shown here.

\subsubsection{Memory of the sample}

For annealing steps with temperatures below the interstitial equilibrium temperature $T_{e q}^{i}$, precipitation occurs [17]. It is obvious that in this case the as-grown state (or other initial states) have an influence on the resulting distribution of the iron concentration. This work will show that the history of temperature treatments of a sample has also to be taken into account, if the annealing temperature lies below the equilibrium temperature $T_{e q}^{\text {tot }}$, at which the total iron concentration in the sample equals the solubility. In this case the as-grown state (or other previous temperature treatments) of a sample is not totally dissolved during annealing and can have an influence on the resulting interstitial iron concentration distribution, as will be shown in section 5.3.1. Consistently, an influence of the as-grown state on results of gettering experiments on neighbor wafers has also been proposed by Liu et al. [24] and an influence of the interstitial iron concentration on the amount accumulated at grain boundaries has been reported by Liu et al. [80] and Haarahiltunen et al. [77]. 


\subsection{Minority carrier lifetime, diffusion length and apparent concentration}

A common method to experimentally determine the interstitial iron concentration of a silicon sample for photovoltaic applications is to calculate the minority carrier diffusion length from lifetime measurements, e.g. photoluminescence imaging (PL), or from radiation induced methods, e.g. light beam induced current (LBIC). From the diffusion length, the apparent interstitial iron concentration can be calculated, assuming that the interstitial iron concentration is the limiting factor for the diffusion length.

The characteristic lifetime $\tau$ of charge carriers in the silicon crystal is the time after which they recombine. There are several recombination mechanisms, like Shockley-Read-Hall recombination, Auger recombination and radiative recombination.

The sum of the reciprocals of the lifetimes $\tau_{i}$ for all the recombination processes give the reciprocal of the total minority carrier lifetime $\tau_{t o t}$ :

$$
\frac{1}{\tau_{t o t}}=\sum_{i} \frac{1}{\tau_{i}}
$$

At room temperature, the dominant recombination process for an indirect semiconductor, like silicon (for non-degenerate, extrinsic silicon), is the Shockley-Read-Hall recombination, which describes recombination via a defect level in the energy band gap. Extended defects (e.g. grain boundaries) and impurity concentrations introduce recombination active deep levels into the band gap. The recombination activity of the defect depends on the position of the deep level, the capture cross section for electrons/holes, the conductivity type and the doping level of the wafer, the density of excess carriers and the temperature. According to the theory of Shockley and Read [81] the lifetime in the case of p-type extrinsic semiconductor under low-injection conditions at room temperature and a doping concentration $p<10^{15} \mathrm{~cm}^{-3}$ can be written as:

$$
\tau_{S R H}=\frac{1}{\sigma_{n} \cdot<v_{n}>\cdot N_{t}}
$$

with $\sigma_{n}$ the capture cross section of the defects for the minority carriers (i.e. electrons for p-type silicon), $\left\langle v_{n}\right\rangle$ the mean thermal velocity of the minority carriers and $N_{t}$ the density of the defect. For details please refer to Blood and Orton [82]. The recombination rate of the majority carriers depends on the excess minority carrier density, as the minority carriers limit the recombination rate.

During their lifetime $\tau$, the charge carriers move a characteristic diffusion length $L$ in the silicon crystal:

$$
L=\sqrt{D_{n} \tau}
$$

with the diffusion coefficient of minority carriers $D_{n}$ as proportionality constant.

Assuming that the interstitial iron concentration $C_{i}$ limits the diffusion length, i.e. $N_{t} \approx C_{i}$, 
an apparent interstitial iron concentration can be calculated from the diffusion length:

$$
C_{a}=\frac{D_{n}}{L^{2} \cdot<v_{n}>\sigma_{n}}
$$

For the calculation of the apparent concentration from LBIC measurements by Krenckel [42] in section 5.3.1, the capture cross section $\sigma_{n}$ will be assumed to be the capture cross section of FeB pairs because the illumination with the LBIC lasers with a wavelength of 850 $\mathrm{nm}$ and $730 \mathrm{~nm}$ is assumed to be too weak to dissociate a significant amount of FeB pairs, as described in section 2.1. The dissociation rate $\Gamma_{d i s}$ can be calculated from the empirical formula determined from experimental data by Geerligs and Macdonald [83]:

$$
\Gamma_{d i s}=\frac{K_{\text {light }} \cdot G^{2}}{C_{F e B}^{2}}
$$

with the empirical parameter $K_{\text {light }}=5 \cdot 10^{-15} \mathrm{~s}$ and the light generation rate of electrons $G$ which is estimated to $G=1.06 \cdot 10^{2} 0 \mathrm{~s}^{-1} \cdot \mathrm{cm}^{-3}$ for the laser with a wavelength of $850 \mathrm{~nm}$ which is the upper limit of the two laser wavelength. The initial concentration of the FeB pairs is approximated by the total iron concentration in the sample: $C_{F e B}=5 \cdot 10^{13} \mathrm{~cm}^{-3}$. With these values the dissociation rate is:

$$
\Gamma_{d i s}=0.0224 s^{-1}
$$

which is a negligible dissociation rate. 


\subsection{Light beam induced current (LBIC) and electron beam induced cur- rent (EBIC)}

Light beam induced current (LBIC) and electron beam induced current (EBIC) are methods to measure the recombination activity of defects in silicon. Figure 6 shows the setup for an EBIC measurement of a p-type silicon sample. A laser or an electron beam is used to generate excess electron-hole pairs with a generation rate $g$. After generation, the charge carriers diffuse to the contacts and are separated unless they recombine before they reach the contacts. In regions of higher recombination activity, e.g. at grain boundaries or other extended defects, less electric current is measured. By scanning over a sample with the laser/ electron beam, an image is achieved with different contrast.

From the current $I$ measured with LBIC, a diffusion length can be calculated via the formula from Donolato [84] assuming a sample with infinite thickness for which recombination at the back surface has not to be taken into account:

$$
I(x, y)=\frac{C_{L B I C}}{1+\frac{1}{\alpha \cdot L(x, y)}}
$$

with the absorption length $\alpha$ and the diffusion length of the minority charge carriers $L$ at the position (x,y). The constant $C_{L B I C}$ contains experimental conditions like laser power and the contact properties of the sample. If the sample is measured with two lasers of different wavelength, the constants can be fitted by using the least square method. For details how the diffusion length can be calculated from the LBIC currents please refer to Krenckel [42].

In order to model the LBIC current with the finite element method, the minority carrier diffusion equation can be used to describe the density of excess minority carriers in a sample during an LBIC measurement:

$$
-D_{n}(\Delta n(x, y))+\frac{D_{n}}{L^{2}} n(x, y)=g_{n}(x, y)
$$

with the diffusion coefficient for the minority carriers $D_{n}$, the diffusion length $\mathrm{L}$ and the laser

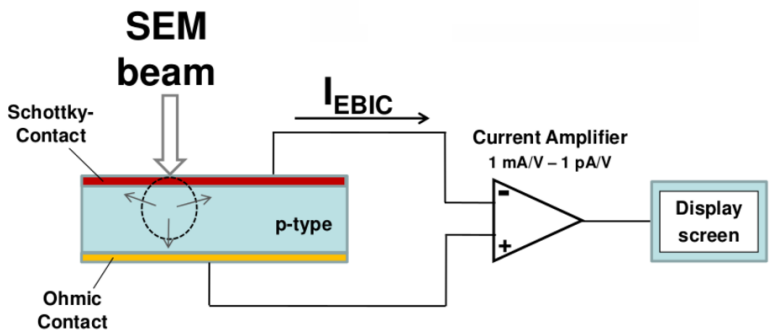

Figure 6: Setup for an EBIC measurement. The silicon sample with a Schottky contact on the front side and an Ohmic contact on the backside is radiated by an electron or a laser beam. The current is measured and displayed as image of light and dark contrasts. 
generation rate $g_{n}$

It is derived from the equations of the drift-diffusion model for electrons/ holes in semiconductors [85] with the assumption of a stationary state during laser radiation and low-level injection and neglecting electrical fields. This equation is used in the LBIC model described in section 4.3

\subsection{Photoluminescence imaging (PL)}

Photoluminescence imaging (PL) is a method to determine the interstitial iron concentration via photoluminescence emission of recombining electron-hole pairs after optical excitation. In the PL measurements by Liu et al. [35] and Liu and Macdonald [53], used for comparison with simulations in this work, the laser used for excitation has a wavelength of 815 $\mathrm{mm}$ [40]. The photoluminescence emission is detected with a CCD camera. The local count rates can be converted into a local excess carrier density $\Delta n$ with a conversion factor of $C_{1}=1 \cdot 10^{10} \frac{\mathrm{cm}^{-3}}{\text { count }}$, determined by Macdonald et al. [40] from comparison with QSSPC (quasi-steady-state photo conductance) measurements. From the local excess carrier density $\Delta n$, the lifetime $\tau$ can be calculated via:

$$
\Delta n=G \cdot \tau
$$

with the laser generation rate $G$. If the photoluminescence emission is measured before and after dissociation of FeB-pairs, i.e. before and after sufficiently long illumination with light, the interstitial iron concentration can be calculated from the lifetimes:

$$
C_{F e i}=C_{2} \cdot\left(\frac{1}{\tau_{1}}-\frac{1}{\tau_{0}}\right)
$$

where $\tau_{1}$ is the lifetime after dissociation and $\tau_{0}$ is the lifetime before dissociation. As described in Macdonald et al. [40], the conversion factor $C_{2}$ depends on the doping concentration in the sample and on the capture cross sections and the energy levels of the iron and FeB states. The authors also describe that for PL measurements possibly not all other recombination channels are eliminated by the subtraction of the two lifetimes as usually assumed for these kind of lifetime measurements. The assumption that the other recombination channels cancel out is only valid if 1.) both lifetime measurements have the same local value for the excess carrier density $\Delta n$, which is usually not the case and 2.) the other recombination channels are not altered by the process of $\mathrm{FeB}$ pair dissociation. However, for cases in which the lifetime is dominated by the iron concentration, the errors arising from other recombination channels can assumed to be small. 


\section{Experimental evidence of impurity accumulation at grain boundaries}

In this section, two examples for the detection of impurity accumulation at grain boundaries are presented after a short introduction of the methods used to detect recombination-active defects experimentally. In this context a method is described which was developed in this work to achieve more information about recombination-active defects with high resolution and high sensitivity. The results which are presented in this section have been published in Falkenberg et al. [43] and Falkenberg and Seibt [71].

\subsection{Experimental methods}

To improve processing schemes and material properties, a fundamental understanding of the physical properties of recombination-active extended defects is presumed. Not only information about the electronic structure and the spatial distribution of these defects is required, but also good knowledge of the chemistry, atomic structure and the origin of recombination-active extended defects.

Electronic structure and spatial distribution can be investigated by methods using radiationinduced secondary signals like LBIC, EBIC or XBIC (x-ray-beam induced current) and it has recently been shown that chemical information of recombination-active defects can be gathered with synchrotron-based $x$-ray fluorescence $(\mu$-XRF), especially in crystalline silicon [10]. Combined with XBIC a high spatial resolution is achieved and a low minimum defect density is needed to detect defects. However, this method is restricted to second phase defects and is insensitive to light elements like carbon, oxygen, nitrogen and no information of the atomic structure is provided.

A new approach is to use the advantages of transmission electron microscopy to obtain information about chemical nature and atomic structure at high resolution in combination with EBIC to localize recombination-active extended defects and FIB techniques to prepare a TEM lamella on-site. This overcomes the disadvantage of conventional TEM lamella preparation which requires a high defect density. An in situ EBIC in a dual beam FIB system guarantees the transfer of the spatial coordinates from defect detection with EBIC to the subsequent TEM lamella preparation in the FIB. Table 2 shows a comparison of the techniques to characterize extended recombination-active defects. It summarizes which information the different techniques can provide, which resolution can be achieved and which minimum defect density is needed to detect defects, i.e. which sensitivity they have. It shows that the combination of TEM with EBIC/FIB preparation provides the desired information of extended recombination-active defects with high resolution and high sensitivity. For more information on the sensitivity and the position accuracy please refer to Falkenberg et al. [43]. 


\begin{tabular}{lcccccc}
\hline \hline Technique & $\begin{array}{c}\text { Recombination } \\
\text { activity }\end{array}$ & $\begin{array}{c}\text { Defect } \\
\text { distri- } \\
\text { bution }\end{array}$ & $\begin{array}{c}\text { Atomic } \\
\text { structure }\end{array}$ & $\begin{array}{c}\text { Chemical } \\
\text { nature }\end{array}$ & $\begin{array}{c}\text { Spatial } \\
\text { resolution } \\
(\mathrm{nm})\end{array}$ & $\begin{array}{c}\text { Sensitivity } \\
(\text { defects/cm }\end{array}$ \\
\hline Defect etching & - & + & - & - & 500 & $10^{3}$ \\
LBIC & + & + & - & - & 5000 & $10^{3}$ \\
EBIC & + & + & - & - & 1000 & $10^{3}$ \\
$\mu$-XRF/XBIC & - & + & - & + & 10 & $10^{3}$ \\
TEM & - & - & + & + & $<0.1$ & $>10^{8}$ \\
TEM/EBIC/FIB & + & + & + & + & 50 & $10^{3}$
\end{tabular}

Table 2: Comparison of different methods for the characterization of extended recombinationactive defects and the information they can provide. Also listed is the resolution that can be achieved and the sensitivity of the method.

\subsection{Sample preparation}

The samples investigated in this section are multicrystalline p-type silicon samples from a nearcap region of block-cast silicon produced in an experimental furnace at Sunicon AG, Freiberg, Germany, for the German research cluster Solar Focus [86]. The block was intentionally contaminated with 20ppma copper and 2ppma iron [87], which resulted in 0.02 ppma iron and 0.4ppma copper in the near-cap region according to neutron-activation-analysis (NAA).

Schottky contacts were prepared with a standard cleaning procedure with organic solvents (acetone and propanol), a subsequent final etching in $H F: H_{3}(1: 10)$ and a dip in diluted HF to remove oxide layers. After keeping the samples on air for $20 \mathrm{~min}$ an Al layer with a thickness $<50 \mathrm{~nm}$ was deposited in the UHV chamber.

For the localization of extended recombination-active defects a standard GATAN EBIC setup with a low-noise Stanford SR570 current amplifier was installed in a FEI Nova NanoLab 600 dual beam Focused Ion Beam (FIB) and Scanning Electron Microscope (SEM) system. The dual beam system also contains a gas injection system for site-specific deposition of Pt with the ion or electron beam from a Pt-organic-precursor gas and a micro manipulator to lift-out small pieces of a sample.

The TEM lamellas are analyzed with a Philips CM200-UT-FEG at 200kV. For X-ray analysis (EDX) the TEM is equipped with a Si:Li detector (Link ISIS system). 


\subsection{TEM lamella preparation}

Figure 7 shows, how a TEM lamella is prepared from a recombination-active extended defect detected at a grain boundary in a multicrystalline silicon sample after localizing it with EBIC. Figure 7(a) shows a high resolution EBIC image of a defect at a grain boundary with a beam voltage of $5 \mathrm{kV}$. The arrow indicates a Pt:C patch which was deposited with the ion beam to protect the features of interest from radiation of the $30 \mathrm{kV} \mathrm{Ga}+$ ion beam during TEM lamella preparation. Figure 7(b) shows the same region in SEM, revealing a needle labeled 'D' which sticks out of a groove at the surface, while the underlying recombination-active features are not visible. Figure $7(\mathrm{c})$ shows the TEM lamella created by removing the material around it with the FIB. The letter ' $\mathrm{C}$ ' labels another needle within the adjacent grain and the arrows indicate the position of the grain boundaries. Figure 7(d) shows a SEM image of the lamella after cutting it out, moving it to a Mo TEM grid with aid of the micro manipulator and thinning it with a $5 \mathrm{kV}$ Ga ion beam. The groove, now filled with Pt:C, can be seen, as well as the needle labeled 'D'. The arrows indicate the position of the grain boundary. 


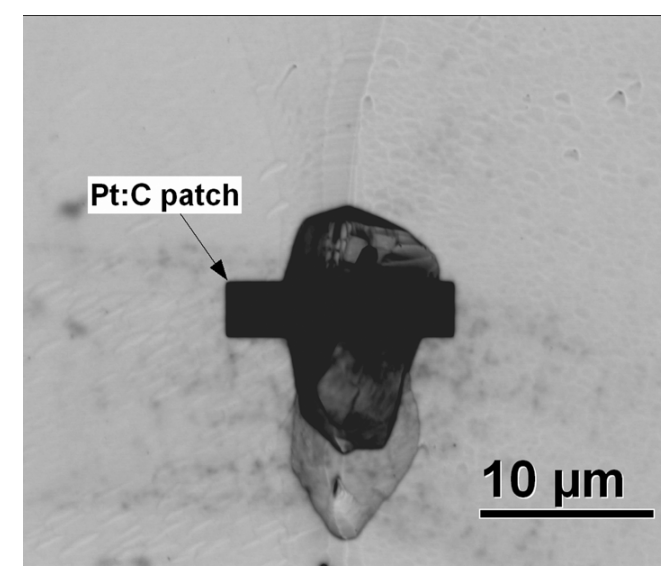

(a) EBIC image of the defect

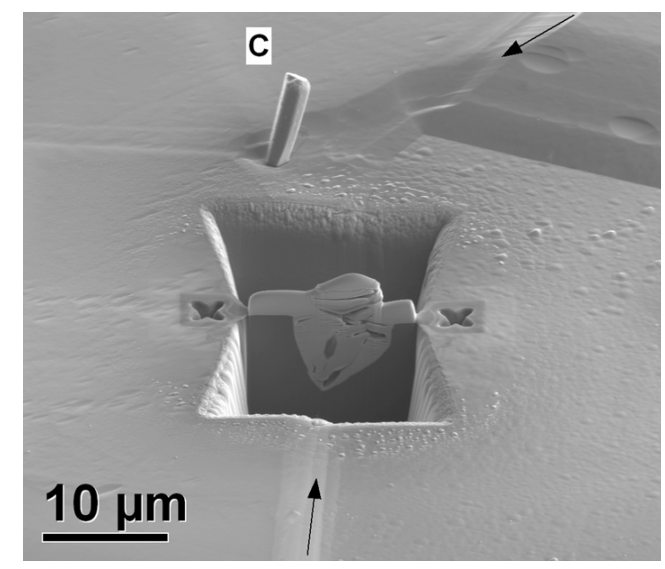

(c) SEM image after removing material

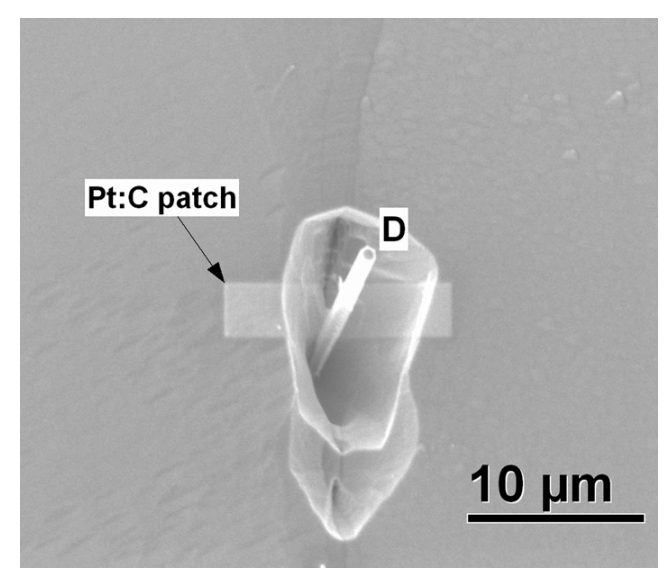

(b) SEM image of the defect

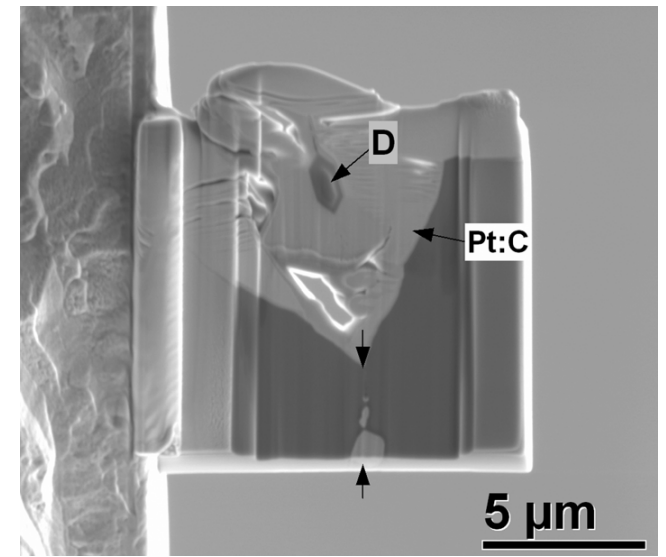

(d) SEM image of the TEM lamella

Figure 7: Illustration of a TEM lamella preparation with the FIB after localizing an extended recombination-active defect with EBIC. Figure 7(a) shows a high resolution EBIC image at 5kV beam voltage of a recombination-active defect at a grain boundary. Figure 7(b) shows the same region in the SEM, revealing a groove and a needle labeled ' $D$ ' at the surface. The rectangle in both figures shows the Pt:C patch which was deposited with the electron beam to protect the region of interest from ion beam radiation during TEM lamella preparation. Figure 7(c) shows a SEM image after material was removed with the ion beam. Figure 7(d) shows a SEM image of the TEM lamella after thinning. 


\subsection{Experimental results}

\subsubsection{Recombination-active defect at a needle-like structure}

Figure 8(a) shows a low magnification EBIC image obtained at a beam voltage of $20 \mathrm{kV}$ of the multicrystalline silicon sample in which the defect in figure 7 was detected. The grain boundaries appear as dark lines and several small recombination active defects in the grain are seen as small dots of dark contrast - one example marked as 'N'. In addition, two large recombination active regions, originating at the grain boundaries and spreading into the adjacent grains, are seen. The region marked by the rectangle is the region shown in figure 7 to explain the sample preparation. The EBIC image in figure 8(b) is an enlarged image of the rectangle and shows the recombination active region in detail. The position of the grain boundaries is indicated by the black arrows. The letters 'A', 'B', and 'C' mark three small recombination active defects in the grains. Figure 8(c) shows a SEM image of the same region. While some features seen in EBIC are also visible in SEM, like the defect labeled ' $C$ ' and the grain boundaries, the defects labeled 'A' and 'B' are not visible on the surface. The SEM image shows the needle and the groove shown in figure 7(b) before depositing the $\mathrm{Pt}$ : $\mathrm{C}$ patch. The butterfly-shaped recombination active area, which spreads out $20 \mu \mathrm{m}$ into the adjacent grains and reveals a dot-like structure at higher magnification (please see figure $8(d)$ is not visible in SEM. 


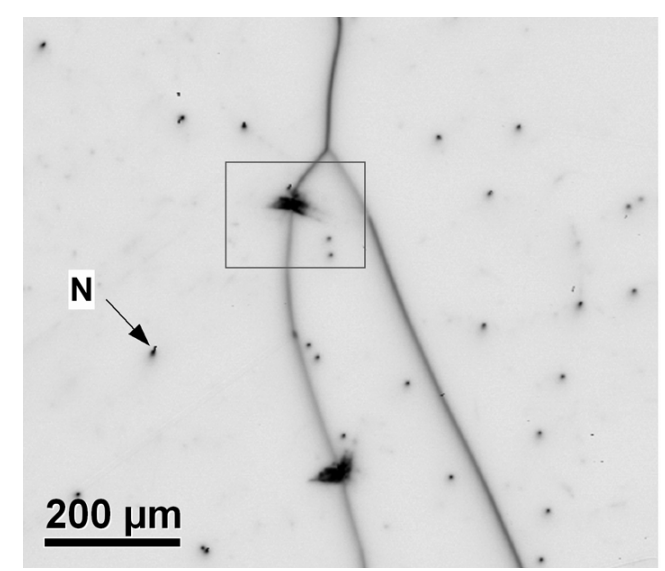

(a) EBIC image at low magnification

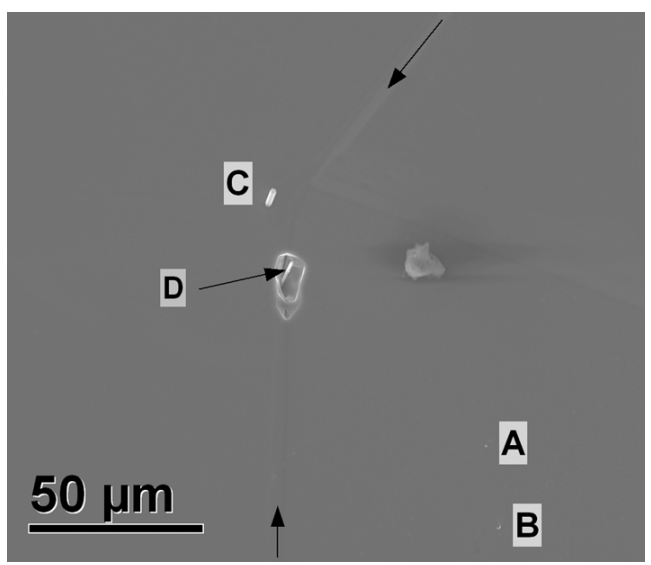

(c) SEM image

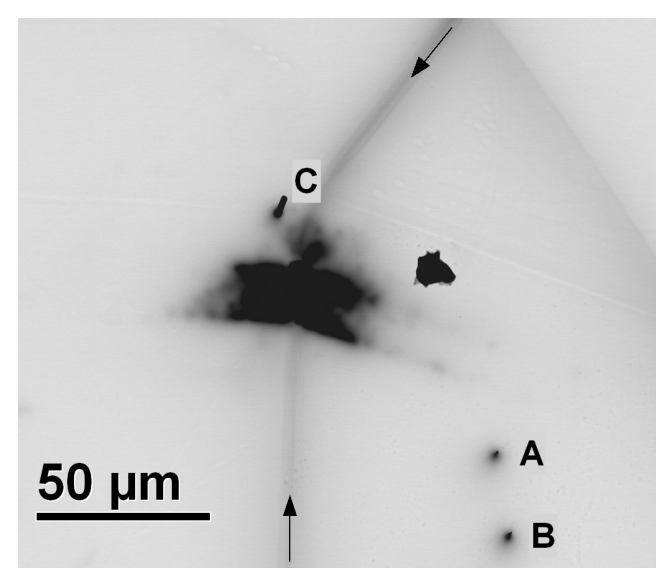

(b) EBIC image of the rectangle in figure $8(\mathrm{a})$

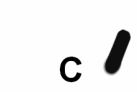

\section{$\underline{20 \mu m}$}
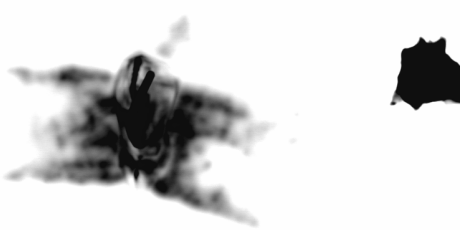

(d) EBIC image at higher magnification

Figure 8: Figure 8(a) shows a low magnification EBIC image at $20 \mathrm{kV}$ beam voltage of a multicrystalline silicon sample. Grain boundaries can be seen as dark lines. There are several small recombination-active defects in the grains (e.g. labeled as ' $N$ ') and two large recombinationactive defects at the grain boundaries. The defect marked by the rectangle is shown in figure 7 to illustrate sample preparation. The EBIC image in figure 8(b) and the SEM image in figure 8(c) show the region marked by the rectangle in figure 8(a). While the SEM image shows the surface features like the groove and the needles, the EBIC image reveals the underlying recombinationactive features. The EBIC image of the extended defect at higher magnification in figure 8(d) reveals a dot-like structure. 
Figure 9(a) shows a STEM annular dark-field image of the TEM lamella at low magnification. The needle is pointed out by the arrow and the grain boundary is indicated by the dotted line. The image clearly shows an extended dark contrast at the grain boundary, which has a diameter of approximately $1 \mu \mathrm{m}$. EDX maps of the region around the dark contrast labelled ' 1 ' reveal an enhanced copper signal as shown in figure 9(b) and a reduced silicon signal as shown in figure 9(c) at the positions of the extended dark contrasts. The EDX line scans shown in figure 9(d) were extracted at the position labeled ' 1 ' and '2' and confirm an enhanced copper signal at the position of the dark contrast and a reduced silicon signal in comparison to the reference line scan at position '2'. Signals for other metal impurities like iron or nickel are not observed.

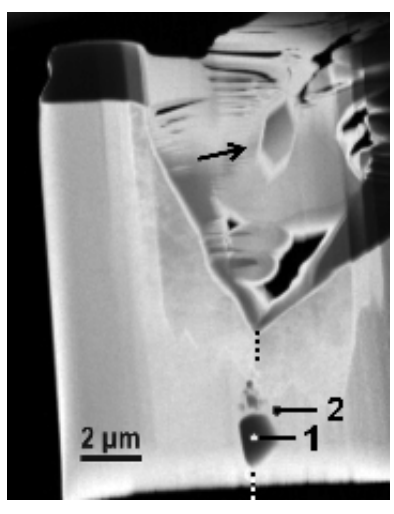

(a) STEM image

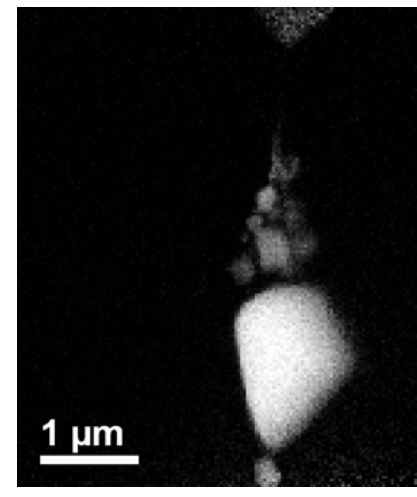

(b) EDX map of copper

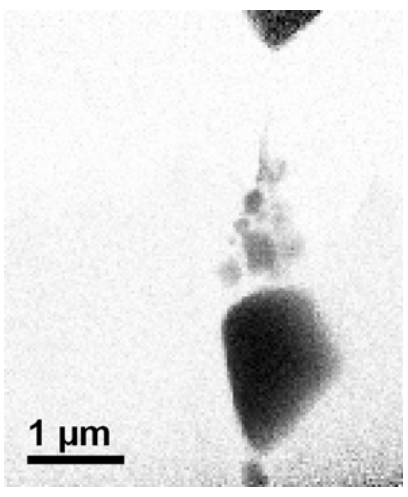

(c) EDX map of silicon

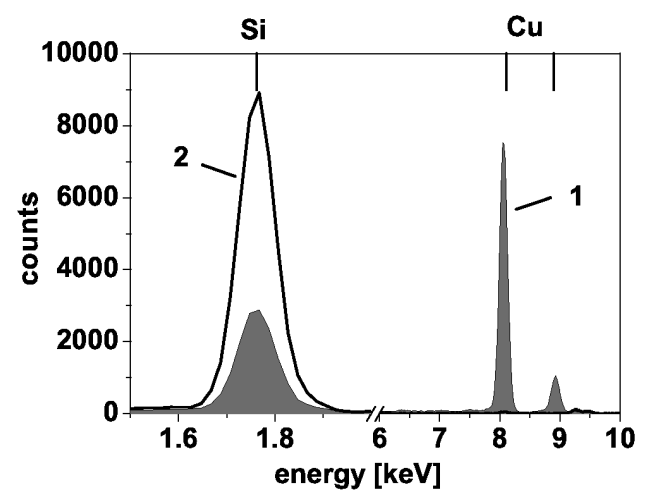

(d) EDX spectra

Figure 9: TEM analysis of the lamella in figure 7. Figure 9(a) shows a STEM annular dark field (ADF) image of the lamella at low magnification with the needle marked by the arrow, the grain boundary marked by the dotted line and several larger dark contrast at the grain boundary, the largest one located around the position '1'. The EDX spectra extracted at the positions ' 1 ' and at the reference position '2' show an enhanced copper signal and a decreased silicon signal at the position '1'. The EDX maps of copper in figure 9(b) and silicon in figure 9(c)] show the region, where the dark contrasts are located and confirm the results from the EDX spectra. 
Figure 10(a) shows a bright-field image of the two grains adjacent to the grain boundary in figure 9(a) with an extensive network of dark lines spreading out from the copper rich dark feature at the grain boundary. The STEM bright-field image with higher magnification of the grain labeled 'grain 2' in figure 10(b) shows dark dot-like contrasts with a size of $10-20 \mathrm{~nm}$ located at the dark lines. The dot-like contrasts -some examples marked by the circles - show an enhanced copper signal in the copper EDX map of the same region in figure 10(c)

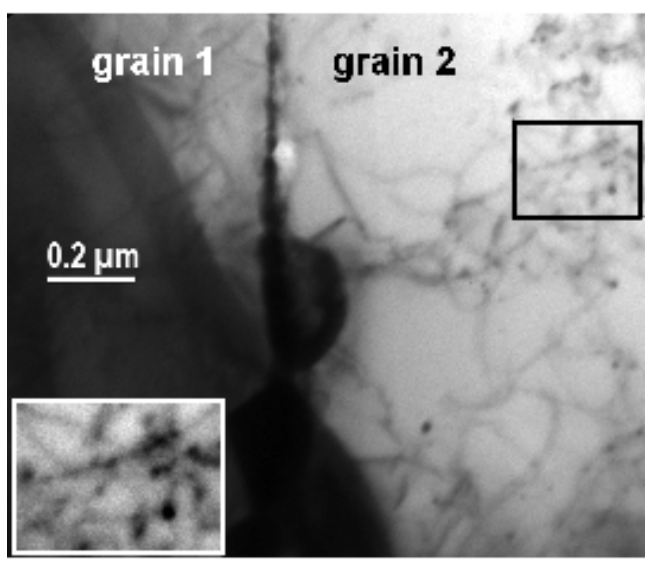

(a) TEM bright-field image

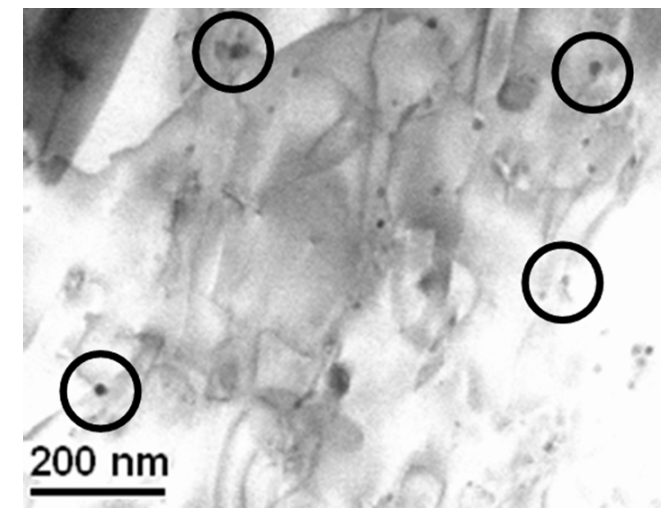
(b) STEM bright-filed image at higher magnification

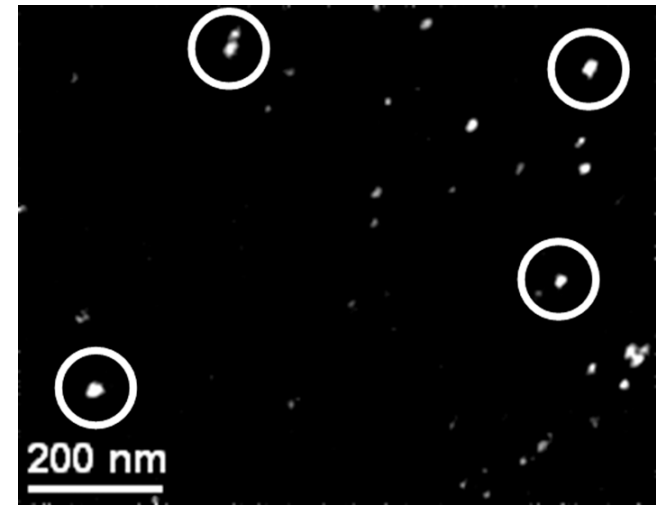

(c) EDX map for copper

Figure 10: The TEM bright-field image in figure 10(a) shows the grain boundary with the two adjacent grains and a network of dark lines. The STEM image in figure 10(b) reveals small dotlike contrasts positioned at some of the dark lines. The EDX map of copper of the same region reveals an enhanced copper signal at the position of the small dots, as indicated by the circles. 


\subsubsection{Recombination-active defect at a honeycomb-like structure}

Figure 11(a) shows an EBIC image of a second sample with a grain boundary and a large recombination active defect of a size of $40 \mu \mathrm{m}$, which originates at the grain boundary and spreads out into the adjacent grains. Also, several smaller spots of high recombination activity at the grain boundary can be seen as indicated by the black arrows. Figure 11(b)] shows the extended recombination active region at a lower beam voltage of $10 \mathrm{kV}$ and reveals a dot-like structure. Figure 11(c) shows a honeycomb-like structure in the central part of the large defect and a very strong recombination active region (marked by the white arrow) at one side of the honeycomblike structure. The dashed rectangle marks the Pt:C patch with the region where a TEM lamella was cut out. Figure 11(d) shows the TEM lamella in the SEM. It reveals a groove filled with Pt:C and a bright contrast at the position of the grain boundary (marked by the circle).

The STEM bright-field image in figure 12(a) shows the two grains adjacent to the grain boundary. They are separated by a dark contrast at the location of the grain boundary. The different appearance of the grains is due to different diffraction conditions in the bright-field. The EDX maps of the same region show a reduced signal for silicon and an increased signal for oxygen and copper at the location of the grain boundary. While the oxygen signal is distributed evenly, the copper signal shows a dotted structure as illustrated also by the EDX line scans in figure 12(b) which were extracted at the position indicated with the white rectangle in figure $12(\mathrm{a})$

The weak beam dark-field image of the grain boundary and 'grain 1' in figure 12(c) shows bright contrasts emanating from the grain boundary region with high oxygen and copper signals. 


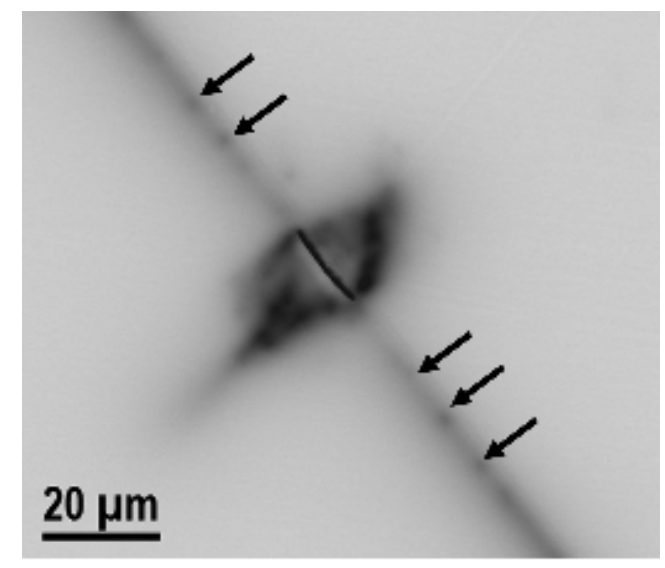

(a) EBIC image

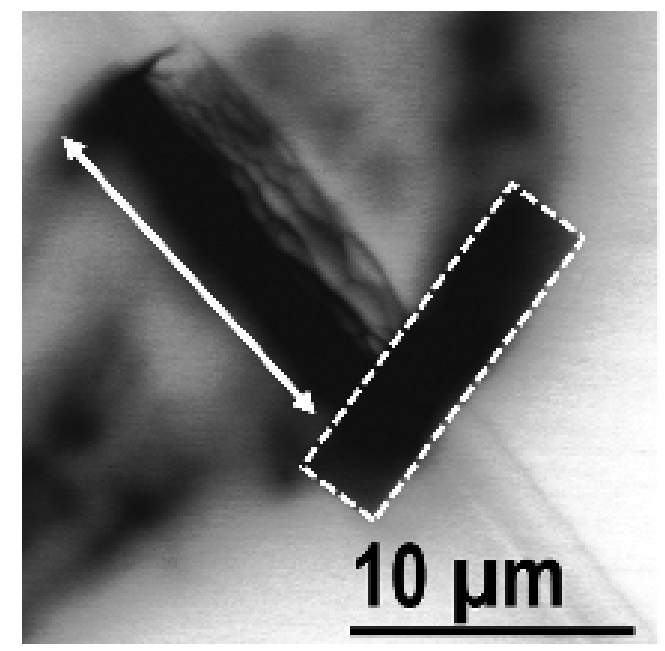

(c) EBIC image of the marked area for TEM lamella preparation

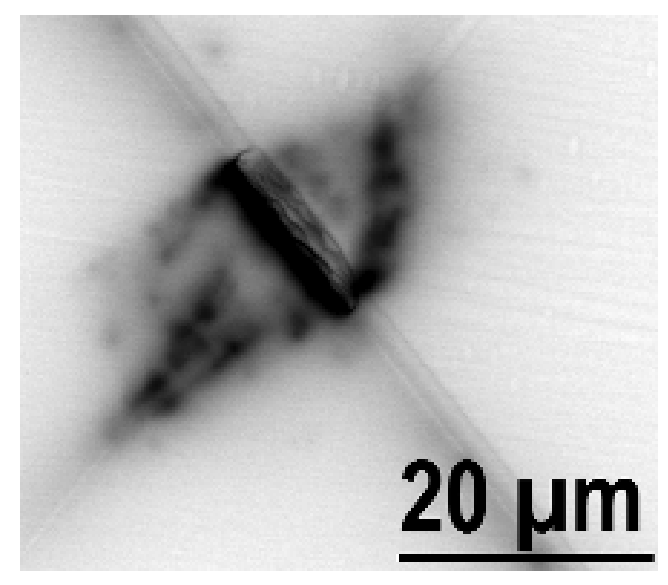

(b) EBIC image at higher magnification

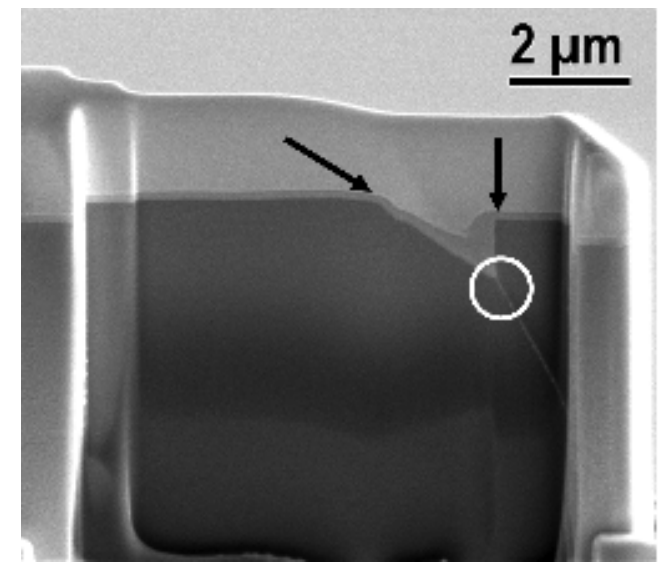

(d) SEM image of the TEM lamella

Figure 11: EBIC and SEM images of a second multicrystalline silicon sample with an extended recombination-active defect located at a grain boundary. Figure 11(a) shows the large defect and the grain boundary as a continuous line of dark contrast with several small dot-like spots of enhanced contrast. Figure 11(b) reveals the dot-like structure of the defect at lower acceleration voltage of $10 \mathrm{kV}$. The EBIC image at higher magnification reveals a honeycomb-like structure and a dark contrast next to it. The TEM lamella shown in figure 11(d) was extracted from the region marked by the white rectangle in figure $11(\mathrm{c})$. 


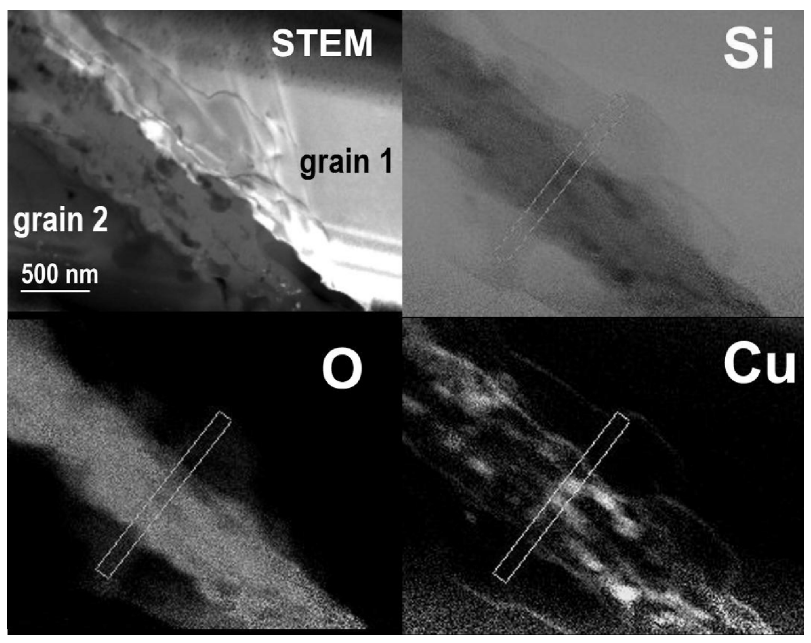

(a) STEM image and EDX maps

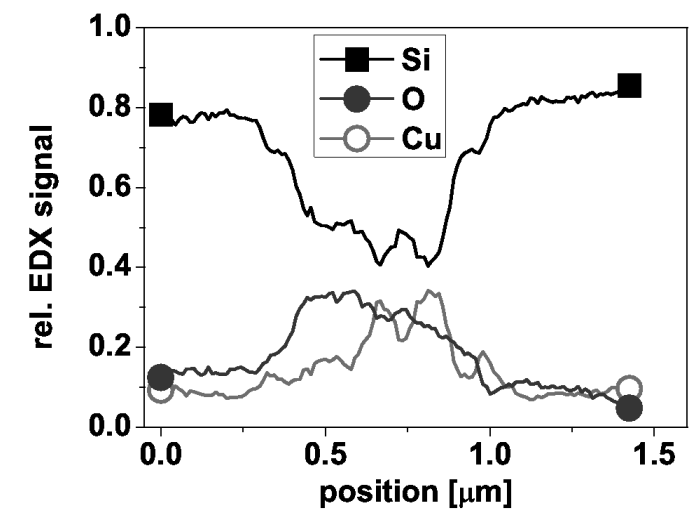

(b) EDX line scans

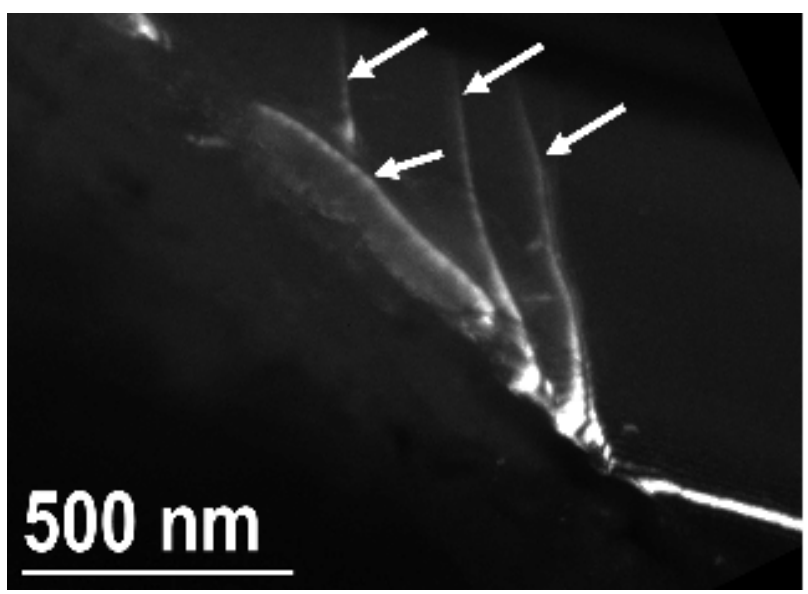

(c) Weak beam dark-field of 'grain 1'

Figure 12: Results of the TEM analysis of the lamella in figure 11(d). Figure 12(a) shows the STEM bright-field image of the grain boundary and the two adjacent grains and the EDX maps for silicon, oxygen and copper of the same region. The rectangle marks the position where the EDX spectra in figure 12(b) were extracted. EDX maps and line scans reveal a decreased silicon signal, a continuous enhanced signal of oxygen and spots of an enhanced copper signal in the grain boundary. The x-ray counts in the EDX spectra in figure 12(b) are normalized, but not corrected for k-factor and bremsstrahlung background. Figure 12(c) shows a weak beam dark-field of 'grain 1' from figure 12(a) with lines of bright contrast emanating from the grain boundary which contains oxygen and copper. 


\subsection{Discussion of the experimental results}

\subsubsection{Accumulation of copper at silicon nitride needles}

The small dots of dark contrast in the grains and sometimes at the grain boundary -one of them marked as ' $\mathrm{N}$ ' in figure 8(a)- proof to be rod-shaped needles, when looking at them in the SEM at higher magnification. Two examples of these needles sticking out of the surface (marked 'D' and ' $\mathrm{C}$ ' in figure 8(c) , while others are only visible in EBIC as can be seen when comparing the position of the dark contrast labeled 'A' and 'B' in figure 8(b) with the EBIC figure of the same region in figure $8(\mathrm{c})$. From electron diffraction it can be deduced that the needles are $\alpha-S_{3} N_{4}$ precipitates which are often found in the upper regions of block-cast multicrystalline silicon. According to Bauer et al. [20] these nitride needles are insulating and show no Ohmic shunts. The groove around the nitride needle labeled ' $\mathrm{D}$ ' in figure 8(c) is an etch pit due to the etching for contact preparation. It is shown at higher magnification in the SEM image in figure 7(b), During TEM lamella preparation it was filled with Pt:C, as can be seen in figure 7(d), The extended dark contrast at the grain boundary in the STEM image of the TEM lamella in figure 9(a) is a copper precipitate and has, according to the EDX line scan in figure $9(\mathrm{~d}) \mathrm{l}$ and the EDX maps in figure 9(b) and 9(c), a composition close to $C u_{3} S i$ as it is expected from the phase diagram [16]. The size of the precipitate is with $1 \mu \mathrm{m}$ extraordinary large. Usually copper precipitates are found in colonies, where planar arranged precipitates with a size of $100 \mathrm{~nm}$ are found [88].

Copper colonies are often surrounded by dislocations incorporating silicon self-interstitials which are emitted during precipitate formation. The large copper precipitate found in this sample is at the origin of an extended network of dislocations observed as dark lines in figure 10(a), The dislocations compensate the huge misfit strain due to the volume expansion of such a large precipitate [89], as described in section 2.1.4.

The dislocation network is decorated with small copper precipitates as can be seen by comparison of the STEM image in figure 10(b) and the EDX map in figure 10(c). These precipitates promote further expansion of the dislocation network. As copper is strongly recombination active [54], the copper precipitates at the dislocation network are most likely the origin of the butterfly-shaped extended region of dark contrast seen in EBIC (see e.g. figure 8(b)).

\subsubsection{Accumulation of copper at silicon oxides}

The TEM analysis of the second sample shows that the bright contrast seen in the SEM image in figure 11(d) and in the TEM image in figure 12(a) is a second phase consisting of a large silicon oxide precipitate and several small copper silicide precipitates. The dark contrast in the EBIC image in figure 11(c) is probably due to the copper silicide precipitates. The honeycomb-like structure observed in the SEM image in figure 11 is typically observed for silicon oxide defects after chemical etching since metal silicides usually produce a localized shallow etch pit. Similar to the observations in the first sample, bright contrast lines are found in the weak beam darkfield TEM image of the grain 1 in figure 12(c). With the results from the first sample, this hints to a dislocation network decorated with small copper silicide precipitates as the origin of the extended dark contrast seen in the EBIC (see e.g. figure 11(b)]. 


\subsection{Conclusion}

The observations give evidence for the accumulation of metal impurities (here: copper) at grain boundaries and show that copper-rich precipitates are found at silicon oxide and silicon nitrides which are located at grain boundaries. The oxygen precipitates covered with copper indicate that smaller oxygen precipitates at grain boundaries also serve as nucleation sites for metal impurities. Even though metal traces are also found at isolated needles by x-ray fluorescence [90], it can be concluded that nitrides at grain boundaries trigger stronger copper precipitation than nitrides in the grains. From the precipitates at the grain boundary, large recombination active regions with a size of up to several tens of microns spread into the adjacent grains. These recombination active regions are due to large dislocation networks, which form due to the strain during precipitation and are decorated with small copper precipitates. This indicates that grain boundaries are not sufficient to compensate the misfit strain due to precipitation. 


\section{Model description}

This section describes the two dimensional model created to simulate the behavior of metal impurities in silicon on wafer-scale for the example of iron. In order to describe diffusion, precipitation, segregation and gettering of a metal impurity, a system of coupled partial differential equations (PDEs) has to be solved. The partial differential equations for the different species of the metal impurity are diffusion equations, which are coupled throughout the sink and source terms. Comsol Multiphysics is used to solve the PDEs via the finite element method (FEM). This PDE solver environment was chosen due to its comfortable user interface, its large choice of PDE solver and solver options and a well established support. We chose to use a two dimensional model to be able to model diffusion and gettering in presence of two dimensional defects like grain boundaries, which cannot be modeled sufficiently with a one dimensional model on the one hand and reduce calculation time in comparison to a three dimensional model on the other hand.

The output of the comsol gettering model is the spatial and temporal evolution of the concentrations of the different species of the metal impurity, namely the interstitial metal impurity concentration in the silicon, the gettered metal impurity concentration and the precipitated and segregated metal impurity concentration at the grain boundary as well as the precipitated metal impurity concentration in the grains. The details of the model will be described in the following section 4.1 and technical details of the implementation will be described in section 4.2 .

The interstitial iron concentration can be converted into a diffusion length via the ShockleyRead-Hall equation as described in section 2.4 and with aid of an LBIC model developed by Nitzbon [91], the recombination effect of a grain boundary on the LBIC current and/ or the diffusion length can be modeled. Details about the implementation of the LBIC model are described in section 4.3 ,

Figure 13(a) shows the geometry of a silicon wafer with one grain boundary. The sample thickness equals the wafer thickness, which typically is between $180 \mu \mathrm{m}$ and $330 \mu \mathrm{m}$. The grain size of multicrystalline silicon ranges from several $\mathrm{cm}$ to the $\mathrm{mm}$ range. The model is set up to have a grain boundary perpendicular to the gettering layer on the backside. In-diffusion possibly takes place from the top.

In the model described below only the $\mathrm{x}-\mathrm{y}$-plane is modeled and symmetry along the z-axis is assumed. The figure 13(b) shows the two dimensional model geometry and numerates the boundaries used in the description of the equations in sections 4.2.1 to 4.2.5. 


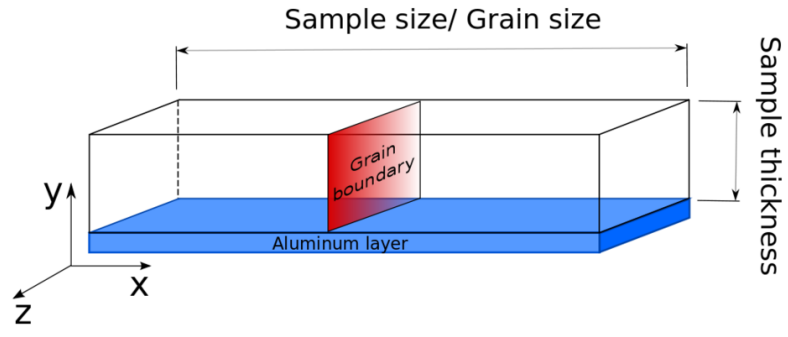

(a) Three dimensional sample geometry

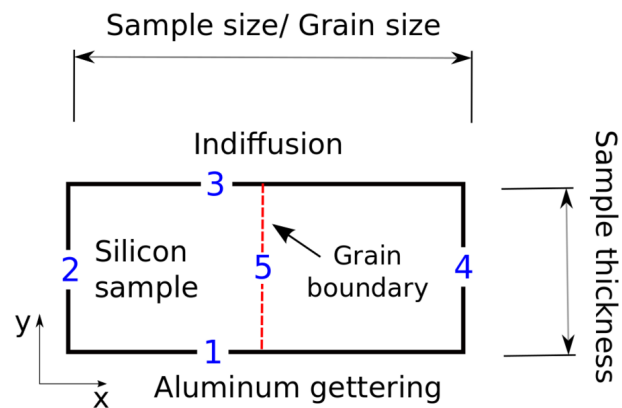

(b) Two dimensional model geometry

Figure 13: Typical simplified sample geometry and the corresponding two dimensional model geometry.

\subsection{Physical description of the model}

The two dimensional model developed in this work is based on the one dimensional model described in Kveder et al. [30] and Seibt et al. [33]. It was extended to include segregation and precipitation as mechanisms of impurity accumulation at a grain boundary. The following sections describe the different mechanisms which are considered: diffusion of metal impurities in the silicon bulk, aluminum gettering (AlG), precipitation in the grain and at the grain boundary and segregation at the grain boundary. Since the focus of the simulations in this model is on iron as metal impurity, this description is confined to this interstitially diffusing metal impurity, and does not include mechanisms that have to be taken into account for predominantly substitutional dissolved impurities such as gold, even though the corresponding mechanisms are included in the one dimensional model.

\subsubsection{Diffusion of metal impurities in the silicon bulk}

For the diffusion of a metal impurity in intrinsic silicon by an interstitial mechanism (as it is the case for iron), the diffusion equation is simply given by Fick's second law:

$$
\frac{\partial C_{S i}}{\partial t}-D\left(\frac{\partial^{2} C_{S i}}{\partial x^{2}}+\frac{\partial^{2} C_{S i}}{\partial y^{2}}\right)=0
$$

In the equation, $C_{S i}$ is the concentration of the interstitial metal impurity in silicon and $D$ is the diffusion coefficient of the interstitial metal impurity in silicon. This notation was chosen because not only the concentration of the metal impurity in silicon, but also the concentration of the metal impurity in the Al:Si liquid and in the grain boundary are calculated in the simulations. Please see section 4.1.7 for the parameters used for the diffusion coefficient of iron. To include precipitation in the grain, a sink and source term has to be added on the right hand side of 
the equation, as described in section 4.1.3, while $\mathrm{AlG}$ and accumulation at the grain boundary are included via boundary conditions. The two dimensional model also includes the following boundary condition for in-diffusion of iron from an infinite source from the front side of the sample:

$$
C_{S i}=C_{e q}=C_{L} \cdot \exp \left(\frac{\Delta S \cdot T-\Delta H}{k T}\right)
$$

with the temperature dependent solubility of the metal impurity in silicon $C_{e q}$ as described in section 2.1.1. (For the parameters used for iron the solubility of iron in silicon please refer to section 4.1.7.)

\subsubsection{Aluminum gettering (AIG)}

As described in section 2.2, aluminum gettering is a segregation mechanism, in which the metal impurities are redistributed to an Al:Si liquid. The Al:Si liquid forms during annealing from the deposited aluminum back surface contact. AlG can simply be implemented by a boundary condition for the flux $F_{A l}$ of the interstitial metal impurities, which is [92, 93]:

$$
F_{A l}=\frac{D}{\lambda}\left(C_{S i}-C_{A l} \cdot \frac{1}{S_{A l}^{*}}\right)
$$

with the concentration of the interstitial metal impurity $C_{S i}$ in silicon, the gettered metal impurity in the Al:Si liquid $C_{A l}$, the atomic jump distance between silicon and the Al:Si liquid $\lambda$ and the diffusion coefficient of the interstitial impurity in silicon $D . S_{A l}^{*}$ is the segregation coefficient for the metal impurity atoms between the silicon bulk and the Al:Si liquid. As described in section 4.1.2, in this model the experimentally determined segregation coefficient of iron:

$$
S_{A l}^{*}=a \cdot \exp \left(\frac{b}{k \cdot T}\right)
$$

measured by Abdelbarey et al. [39] is used. The values for $a, b, \lambda$ and $D$ used for iron are given in section 4.1.7. In order to calculate the metal impurity concentration in the Al:Si liquid for each time step, the flux is integrated over time and divided by the thickness of the Al:Si liquid in the one dimensional model. In the two dimensional model, the following one dimensional PDE is implemented on the boundary in order to consider diffusion into the $\mathrm{x}$-direction:

$$
\frac{\partial C_{A l}}{\partial t}-\left(D_{A l} \frac{\partial^{2} C_{A l}}{\partial x^{2}}\right)=\frac{D}{\lambda} \cdot\left(C_{S i}-\frac{1}{S_{A l}^{*}} \cdot \frac{C_{A l}}{y_{A l}}\right)
$$

with the thickness of the aluminum layer $y_{A l}$. The concentration of the metal impurity in the $\mathrm{Al}$ :Si liquid $C_{A l}$ in the two dimensional model is given by the integral of the three dimensional concentration of the metal impurity in the Al:Si liquid $C_{A l}^{3 d}$ :

$$
C_{A l}(x)=\int_{0}^{y_{A l}} C_{A l}^{3 d}(x, y) d y
$$


and has the unit $\mathrm{cm}^{-2}$.

In both approaches, for the one and the two dimensional model, it is assumed that the diffusion in the Al:Si liquid is very fast and therefore the concentration is uniformly distributed.

\subsubsection{Precipitation in the grain}

In order to take precipitation of the interstitial metal impurities to pre-existing precipitation sites (heterogeneous precipitation due to dislocations, point defects and other impurity atoms e.g. oxygen as described in section 2.1) into account, the time-dependent concentration of precipitated metal impurities $C_{p}$ has to be calculated and a sink and source term $F_{p}=-\frac{\partial C_{p}}{\partial t}$ has to be added to the right hand side of the diffusion equation of the interstitial metal impurities (see equation 15).

The concentration of precipitated metal impurities $C_{p}$ is calculated in the model via a meanfield approximation (see e.g. Balluffi et al. [94]) and by averaging over the size-distribution function of the precipitates:

$$
\frac{\partial C_{p}}{\partial t}=\tau_{p} \cdot\left(C_{S i}-C_{e q}\right)
$$

with the time constant $\tau_{p}$ and the solubility of the impurity in silicon $C_{e q}$ with the assumption of the equilibrium with an infinitively large precipitate.

For spherical precipitates and a diffusion-limited precipitation, the time constant $\tau_{p}$ is given by:

$$
\tau_{p}=4 \pi \cdot D \cdot r_{p} \cdot N_{p}
$$

with the diffusion coefficient of the interstitial impurity in silicon $D$, the effective precipitate density $N_{p}$ and the precipitate radius $r_{p}$. (For the derivation of the formula for the time constant $\tau_{p}$, please refer to e.g. Flynn [95] chapter 10.) Equation 21, with the time constant as given in equation 22, is known in literature as Ham's law [32, 29, 96]. In the model the precipitation sites are assumed to be distributed evenly and all precipitates have an average radius, i.e. a mono-disperse size-distribution is used and Ostwald ripening of the precipitates is not taken into account. Diffusion-limited precipitation is valid for large precipitate radii, while for small precipitate radii precipitation becomes reaction-limited due to a significant contribution from surface energy change (due to incorporation of impurity atoms into the precipitate) and reaction energy to the chemical potential of the impurity atoms. To take these contributions into account the equations 21 and 22 are modified to:

$$
\frac{\partial C_{p}}{\partial t}=\tau_{p}^{*} \cdot\left(C_{S i} \cdot P_{s}-C_{e q}\right)
$$

and

$$
\tau_{p}^{*}=4 \pi \cdot D \cdot r_{p}^{2} \cdot N_{p} \cdot \frac{P_{r b}}{\lambda_{2}+r_{p} P_{r b} P_{s}}
$$


with the diffusion jump length $\lambda_{2}$ for an iron atom regarding the silicon bulk and a precipitate, the contribution of the surface energy to the chemical potential of the impurity atoms $P_{s}$ and the probability $P_{r b}$ for an atom to overcome the reaction energy barrier to join the precipitate.

The contribution $P_{s}$ from the surface energy $E_{s}$ is given by:

$$
P_{s}=\exp \left(\frac{-E_{s}}{k T}\right)
$$

The contribution of the surface energy $E_{s}$ to the chemical potential according to the GibbsThomson equation is (see e.g. Gottstein [49]:

$$
E_{s}=\frac{2 \cdot \Omega \cdot \sigma}{r_{p}}
$$

with the volume of metal atoms in the precipitate phase $\Omega$ and the specific surface energy change per surface $\sigma$.

The probability $P_{r b}$ to overcome the reaction barrier $E_{r b}$ is:

$$
P_{r b}=\exp \left(\frac{-E_{r b}}{k T}\right)
$$

For the values used in the model for iron as metal impurity please refer to section 4.1.7. If $E_{r b}$ and $E_{s}$ are small, the limiting factor is the diffusion in the vicinity of the precipitate and equation 39 reduces to the diffusion-limited equations 21 and 22. A change in strain energy with precipitate size is not included in the model.

The precipitate radius $r_{p}$ is calculated from the density of precipitates $N_{p}$ and the concentration of precipitated metal impurity atoms $C_{p}$ :

$$
r_{p}=\left(\frac{3}{4 \pi} \frac{C_{p}}{C_{L} N_{p}}\right)^{1 / 3}
$$

with the approximation that the concentration of metal impurities in the precipitates is equal to the concentration of lattice sites $C_{L}$.

\subsubsection{Precipitation at the grain boundary}

Precipitation at the grain boundary is modeled as plane with a precipitate density usually exceeding the precipitate density in the grains. The calculation for the time dependent concentration of precipitated metal impurities at the grain boundary is implemented in analogy to precipitation in the grains, with the difference that the concentration of precipitated metal at the grain boundary $C_{g b}$ is calculated by a one-dimensional PDE, which is defined on the grain boundary plane:

$$
C_{g b}(x)=\int_{0}^{x_{g b}} C_{g b}^{3 d}(x, y) d x
$$

with the thickness of the grain boundary $x_{g b}$. The concentration of precipitated metal at the grain boundary $C_{g b}$ has therefore the unit $\mathrm{cm}^{-2}$. It follows that the precipitate density at the grain boundary $N_{g b}$ is also two-dimensional and the time constant $\tau_{g b}$ has the unit $\frac{m}{s}$. 
The flux towards the precipitates at the grain boundary is, in contrast to the grain precipitation, not a sink and source term that has to be added to the diffusion equation 15, but is implemented as a boundary condition at the interface of the grain boundary plane and the silicon.

While the reaction barrier for precipitation at the grain boundary is set to the same value as for grain precipitation, the surface energy is set to zero.

\subsubsection{Segregation at the grain boundary}

Segregation at the grain boundary is modeled as plane with higher solubility than the solubility for the metal impurity in the silicon. The sink and source term is implemented in analogy to segregation of the metal impurities due to aluminum gettering as described in section 4.1 .2 with a different segregation coefficient, defining the ratio between the solubility in the silicon and at the grain boundary:

$$
S_{g b}=\frac{C_{g b}^{e q}}{C_{S i}^{e q}}=a \cdot \exp \left(\frac{b}{k \cdot T}\right)
$$

and the thickness $y_{A l}$ of the Al:Si liquid substituted by the thickness of the grain boundary $x_{g b}$, giving the time evolution of concentration segregated at the grain boundary:

$$
\frac{\partial C_{g b}}{\partial t}=\frac{D}{\lambda_{2}} \cdot\left(C_{S i}-\frac{1}{S_{g b}} \cdot \frac{C_{g b}}{x_{G B}}\right)
$$

with the atomic jump distance $\lambda_{2}$ between silicon and the precipitates. The flux towards the grain boundary is $F_{g b}=-\frac{\partial C_{g b}}{\partial t}$ and is implemented as boundary condition at the interface between grain boundary plane and the silicon. 


\subsubsection{Restrictions of the model}

As consequence of the assumptions made in this model, the following restrictions for the application of the simulations occur:

1. The mean-field approximation is only valid if the mean distance between the precipitates $d$ is smaller than the characteristic length scale $l$ of the concentration profiles of interest: $l>d=\left(\frac{1}{N_{p}}\right)^{\frac{1}{3}}$

2. The quasi-steady state, i.e the flux conservation approximation is only valid if the simulation time $t_{\text {sim }}$ is longer than the transition time until the quasi-steady state has established.

3. The model is only valid for thin Al:Si liquid thicknesses, as it is assumed that the metal impurity in the Al:Si liquid is distributed uniformly.

4. It is controversial, which value for the thickness of the grain boundary has to be used. This influences the simulations with segregation at the grain boundary. It does not influence the simulations for precipitation at the grain boundary since the two-dimensional precipitate density at the grain boundary is a fitting parameter.

In this work a thickness of $10 \AA$ is used, which is suggested from molecular dynamics simulations of the interaction of grain boundaries with intrinsic point defects and carbon in silicon by Käshammer and Sinno [97].

5. The segregation coefficient of grain boundaries are not known. Therefore, quantitative simulations for segregation at a grain boundary are not possible. This will be subject of section 5.5 . 


\subsubsection{Used parameters}

The following table 3 lists the parameters needed for the model, the values used for iron in this work with references and a short description of the parameters.

The values for the initial concentrations $C_{S i}, C_{A l}, C_{p}$ and $C_{g b}$ depend on the simulations. Often a total concentration is known from e.g. NAA measurements. The amount precipitated can then be calculated using the solubility assuming an equilibrium state at the initial temperature. The concentration in $C_{A l}$ is usually assumed to be zero. The ratio of concentration precipitated at the grain boundary and in the grain is usually unknown and can only be derived from preceding simulations. The precipitate densities in the grain $N_{p}$ and at the grain boundary $N_{g b}$ are usually derived by fitting the simulations to experimental data. No reliable data exists for the segregation coefficient for iron regarding the silicon matrix and the grain boundary $S_{g b}$. Most likely it is not a single value for all grain boundaries, but varies with grain boundary type and possibly with contamination level of the grain boundary. For the thickness of the grain boundary a value proposed as result of molecular dynamics simulations of the interaction of grain boundaries with intrinsic point defects and carbon in silicon by Käshammer and Sinno [97] is used. 


\begin{tabular}{|c|c|c|c|}
\hline Parameter & Value & Description & Reference \\
\hline$D$ & $D_{0} \cdot \exp \left(-H^{m} /(k T)\right)$ & Diffusion coefficient of $\mathrm{Fe}$ in $\mathrm{Si}$ & [51] \\
\hline$D_{0}$ & $9.5 \cdot 10^{-4} \mathrm{~cm}^{2} / \mathrm{s}$ & Prefactor for diffusion coefficient $\mathrm{D}$ & [51,47] \\
\hline$\Delta H^{m}$ & $0.65 \mathrm{eV}$ & Migration enthalpy of $\mathrm{Fe}$ in $\mathrm{Si}$ & {$[51,47]$} \\
\hline$C_{e q}$ & $C_{L} \cdot \exp ((T \cdot \Delta S-\Delta H) /(k T))$ & Solubility of $\mathrm{Fe}$ in $\mathrm{Si}$ & [51] \\
\hline$\overline{C_{L}}$ & $5 \cdot 10^{22} \mathrm{~cm}^{-3}$ & Density of interstitial sites in $\mathrm{Si}$ & [51] \\
\hline$\Delta S$ & $8.2 \cdot k$ & Solution entropy of $\mathrm{Fe}$ in $\mathrm{Si}$ & {$[51,46]$} \\
\hline$\Delta H$ & $2.94 \mathrm{eV}$ & Solution Enthalpy of $\mathrm{Fe}$ in $\mathrm{Si}$ & {$[\overline{51}, \overline{46}$} \\
\hline$\lambda$ & $0.543 n \mathrm{~m}$ & $\begin{array}{l}\text { Atomic jump distance of } \mathrm{Fe} \text { from } \mathrm{Si} \text { to } \mathrm{Al}: \mathrm{Si} \\
\text { liquid }\end{array}$ & \\
\hline$S_{A l}^{*}$ & $a \cdot \exp (b /(k T))$ & $\begin{array}{l}\text { Segregation coefficient of Fe regarding Si and } \\
\text { Al:Si liquid }\end{array}$ & [39] \\
\hline$a$ & 1.87 & Prefactor of the segregation coefficient $S_{A l}^{*}$ & [39] \\
\hline$b$ & $1.29 \mathrm{eV}$ & $\begin{array}{l}\text { Exponential factor of the segregation coeffi- } \\
\text { cient }\end{array}$ & [39] \\
\hline$D_{A l}$ & $D_{0, A l} \cdot \exp \left(-H_{A l}^{m} /(R T)\right)$ & Diffusion coefficient of $\mathrm{Fe}$ in $\mathrm{Al}$ & [98] \\
\hline$D_{0, A l}$ & $2.34 \cdot 10^{7} \mathrm{~m}^{2} / \mathrm{s}$ & Prefactor for diffusion coefficient $D_{A l}$ & [98] \\
\hline$\Delta H_{A l}^{m}$ & $35 \mathrm{~kJ} / \mathrm{mol}$ & Migration enthalpy of $\mathrm{Fe}$ in $\mathrm{Al}$ & [98] \\
\hline$\lambda_{2}$ & $0.543 n m$ & $\begin{array}{l}\text { Atomic jump distance of interstitial iron from } \\
\text { Si into precipitate }\end{array}$ & \\
\hline$E_{s}$ & $(2 \cdot \sigma \Omega) / r_{p}$ & $\begin{array}{l}\text { Contribution from surface energy of precipi- } \\
\text { tates }\end{array}$ & \\
\hline$\sigma$ & $7.5 \mathrm{ev} / \mathrm{cm}^{2}$ & Specific surface energy change per surface & \\
\hline$\Omega$ & $2 \cdot 1.85 \cdot 10^{-23} \mathrm{~cm}^{3}$ & Volume of atoms in the precipitate phase & \\
\hline$E_{r b}$ & $0.3 e V$ & Reaction barrier for precipitation & \\
\hline$N_{p}$ & fitting parameter & Precipitate density in the grain & \\
\hline$N_{g b}$ & fitting parameter & Precipitate density at the grain boundary & \\
\hline$S_{g b}$ & fitting parameter & $\begin{array}{l}\text { Segregation coefficient for } \mathrm{Fe} \text { regarding } \mathrm{Si} \\
\text { matrix and grain boundary }\end{array}$ & \\
\hline$x_{g b}$ & $10 \AA$ & Thickness of grain boundary & [97] \\
\hline
\end{tabular}

Table 3: List of parameters with the values used in the simulation model for iron as metal impurity. 


\subsection{Technical description of the model}

In this section the PDEs are listed with their corresponding boundary conditions as they are implemented in Comsol.

\subsubsection{PDE for interstitial metal impurities}

The two dimensional PDE for the diffusion of the interstitial component of the metal impurity in silicon is:

$$
\frac{\partial C_{S i}}{\partial t}-\nabla \cdot\left(D \nabla C_{S i}\right)=-\tau_{p}^{*} \cdot\left(C_{S i} \cdot P_{s}-C_{e q}\right)
$$

The term on the right hand side of the equation describes the source and sink term due to precipitation of the metal impurity in the grain of the sample explained in detail in section 4.1.3.

The boundary conditions for boundaries 2 and 4 (see figure 13(b)) for the interstitial component are zero flux conditions:

$$
-\vec{n} \cdot\left(-D \nabla C_{S i}\right)=0
$$

where $\vec{n}$ describes the normal vector perpendicular to the boundary plane. On boundary 3 on the top, Dirichlet condition describe in-diffusion of the metal impurity from an infinite source:

$$
C_{S i}=C_{e q}
$$

On boundary 1 flux/source boundary conditions (=Robin boundary condition) describe the metal impurity flux into the Al:Si liquid due to gettering. This boundary condition is described in detail in section 4.1.2.

$$
-\vec{n} \cdot\left(-D \nabla C_{S i}\right)=\frac{D}{\lambda} \cdot\left(\frac{1}{S_{A l}^{*}} \cdot \frac{C_{A l}}{y_{A l}}-C_{S i}\right)
$$

Boundary 5 is an additional boundary within the domain representing the grain boundary. Depending on the mechanism for accumulation of the metal impurity at the grain boundary, there are two different boundary conditions implemented which can be used separately or in combination. For precipitation of metal impurities at the grain boundary, the following Robin boundary condition is implemented:

$$
-\vec{n} \cdot\left(-D \nabla C_{S i}\right)=-\tau_{g b}^{*} \cdot\left(C_{S i} \cdot P_{s}-C_{e q}\right)
$$

The boundary condition for segregation of metal impurities is:

$$
-\vec{n} \cdot\left(-D \nabla C_{S i}\right)=\frac{D}{\lambda_{2}} \cdot\left(\frac{1}{S_{g b}} \cdot \frac{C_{g b}}{x_{G B}}-C_{S i}\right)
$$

Both equations are described in detail in section 4.1.4 and 4.1.5. 


\subsubsection{PDE for metal impurities in the Al:Si liquid}

Aluminum gettering, which is described in section 4.1.2, is implemented as one dimensional boundary condition for the concentration of interstitial metal impurities $C_{S i}$ (see equation 35 in section 4.2.1) and as one dimensional PDE defined on boundary 1 (see figure 13(b)] to calculate the time evolution of the metal impurity concentration in the Al:Si liquid. The one dimensional approach is used to avoid meshing problems due to the different length scales of the sample thickness and the Al:Si liquid thickness. The diffusion into y-direction is assumed to be instantaneously, since the diffusion coefficient of the metal impurity in the Al:Si liquid is very high compared to the diffusion coefficient of the metal impurity in silicon and the thickness of the $\mathrm{Al}: \mathrm{Si}$ liquid is small. The equation for the metal impurity concentration in the $\mathrm{Al}: \mathrm{Si}$ liquid $C_{A l}$ is:

$$
\frac{\partial C_{A l}}{\partial t}-\nabla \cdot\left(D_{A l} \nabla C_{A l}\right)=\frac{D}{\lambda} \cdot\left(C_{S i}-\frac{1}{S_{A l}^{*}} \cdot \frac{C_{A l}}{y_{A l}}\right)
$$

\subsubsection{PDE for precipitation in the grains}

The equation for the time evolution of the concentration of the precipitated metal impurities $C_{p}$ in the grain is:

$$
\frac{\partial C_{p}}{\partial t}=\tau_{p}^{*} \cdot\left(C_{S i} \cdot P_{s}-C_{e q}\right)
$$

with $\tau_{p}^{*}$ according to equation 24 in section 4.1 .3

The contribution from the surface energy to the chemical potential $E_{s}$ to calculate $P_{s}$ is:

$$
E_{s}=\frac{2 \sigma \Omega \cdot r_{\text {min }} \cdot \sqrt{2}}{\sqrt{r_{\text {min }}^{2}+r_{p}^{2}}}=\frac{E_{s 0} \cdot r_{\text {min }} \cdot \sqrt{2}}{\sqrt{r_{\text {min }}^{2}+r_{p}^{2}}}
$$

where $r_{\min }$ is the minimal radius allowed for the precipitates. This parameter has to be used to avoid singularities during modeling.

The boundary conditions for the concentration of precipitated impurities for all boundaries 1-5 (see figure 13(b) $)$ have to be set to zero flux conditions:

$$
-\vec{n} \cdot\left(-D \nabla C_{p}\right)=0
$$

\subsubsection{PDE for precipitation at the grain boundary}

The time evolution of the concentration of metal impurities precipitated at the grain boundary $C_{g b}$, as described in detail in section 4.1.4, is a one-dimensional equation defined on boundary 5:

$$
\frac{\partial C_{g b}}{\partial t}=\tau_{g b}^{*} \cdot\left(C_{S i} \cdot P_{s}-C_{e q}\right)
$$


Please note that the concentration $C_{g b}$ has the unit $\mathrm{cm}^{-2}$. In analogy to grain precipitation in section $4.2 .3, \tau_{g b}^{*}$ is the time constant given by:

$$
\begin{gathered}
\tau_{g b}^{*}=\frac{4 \pi D \frac{N_{g b}}{\lambda_{2}} r_{g b}^{2} P_{r b}}{1+\frac{r_{g b}}{\lambda_{2}} P_{r b} P_{s}} \\
{\left[\tau_{g b}^{*}\right]=\frac{m}{s}}
\end{gathered}
$$

The radius of precipitates at the grain boundary $r_{g b}$ is calculated in analogy to section 4.1.3 from the density of precipitates at the grain boundary $N_{g b}$ and the concentration of metal impurity atoms precipitated at the grain boundary $C_{g b}$ :

$$
r_{g b}=\left(\frac{3}{4 \pi} \frac{C_{g b}}{C_{L} N_{g b}}\right)^{1 / 3}
$$

\subsubsection{PDE for segregation at the grain boundary}

The PDE for the time evolution of segregated metal impurities at the grain boundary, as described in section 4.1.5, is given by:

$$
\frac{\partial C_{g b}}{\partial t}=\frac{D}{\lambda_{2}} \cdot\left(C_{S i}-\frac{1}{S_{g b}} \cdot \frac{C_{g b}}{x_{G B}}\right)
$$

\subsubsection{Geometry and mesh}

Some problems occur, when it comes to meshing the present geometry into elements (triangles or rectangles) for applying the finite element method (FEM). In order to make sure that the FEM approximates the solution accurately, the elements of the mesh have to be equilateral or at least nearly equilateral. At the same time, it is necessary to keep the number of elements as small as possible to keep computation time small. It follows that the ratio of the sides, i.e. the width and thickness of the sample has to be $\approx 1$. However, this is not the case for the present geometry with width in centimeter range and thickness in millimeter or even micrometer range. The solution to avoid unequal geometry chosen here is to non-dimensionalize the PDEs with respect to the spatial variables and thus apply the finite element method to a non-dimensionalized set of equations with anisotropic diffusion coefficients. The anisotropic diffusion coefficient for interstitial iron in silicon is then:

$$
D^{*}=\left[\begin{array}{cc}
\frac{D}{L_{y}^{2}} & 0 \\
0 & \frac{D}{L_{x}^{2}}
\end{array}\right]
$$

and the anisotropic diffusion coefficient for interstitial iron in aluminum is:

$$
D_{A l}^{*}=\left[\begin{array}{cc}
\frac{D_{A l}}{L_{y}^{2}} & 0 \\
0 & \frac{D_{A l}}{L_{x}^{2}}
\end{array}\right]
$$


where $L_{y}$ and $L_{x}$ are the scaling parameters for the geometric variables in x- and y-direction respectively. Usually they are chosen so that $L_{y}$ equals the thickness and $L_{x}$ equals the width/ grain size of the sample.

The original equations which contain a spatial variable are the PDE for iron diffusion in silicon (equation 32) with its zero flux boundary conditions (equation 33), the PDE and the boundary conditions for aluminum gettering (equation 35, 38) and the boundary conditions for grain boundary precipitation and segregation (equation 36, 37). These equations transform into the following scaled equations:

The PDE for the metal impurity diffusion in silicon in equation 32 changes to

$$
\frac{\partial C_{S i}}{\partial t}-\nabla^{*} \cdot\left(D^{*} \nabla^{*} C_{S i}\right)=-\tau_{p}^{*} \cdot\left(C_{S i} \cdot P_{s}-C_{e q}\right)
$$

the zero flux boundary conditions in equation 33 become:

$$
-\vec{n}^{*} \cdot\left(-D^{*} \nabla^{*} C_{S i}\right)=0
$$

the PDE for iron in the aluminum gettering layer in equation 38 becomes:

$$
\frac{\partial C_{A l}}{\partial t}-\nabla^{*} \cdot\left(D_{A l}^{*} \nabla^{*} C_{A l}\right)=\frac{D}{\lambda} \cdot\left(C_{S i}-\frac{1}{S_{A l}^{*}} \cdot \frac{C_{A l}}{y_{A l}}\right)
$$

the boundary conditions for aluminum gettering in equation 35 transform to:

$$
-\vec{n}^{*} \cdot\left(-D^{*} \nabla^{*} C_{S i}\right)=\frac{1}{L_{y}} \cdot \frac{D}{\lambda} \cdot\left(\frac{1}{S_{A l}^{*}} \cdot \frac{C_{A l}}{y_{A l}}-C_{S i}\right)
$$

and the boundary conditions for grain boundary precipitation (equation 36 ) and segregation (equation 37) become:

$$
\begin{aligned}
& -\vec{n}^{*} \cdot\left(-D^{*} \nabla^{*} C_{S i}\right)=-\frac{1}{L_{x}} \tau_{g b}^{*} \cdot\left(C_{S i} \cdot P_{s}-C_{e q}\right) \\
& -\vec{n}^{*} \cdot\left(-D^{*} \nabla^{*} C_{S i}\right)=\frac{1}{L_{x}} \frac{D}{\lambda_{2}} \cdot\left(\frac{1}{S_{g b}} \cdot \frac{C_{A l}}{y_{A l}}-C_{S i}\right)
\end{aligned}
$$

with

$$
\nabla^{*}=\left[\begin{array}{c}
\frac{\partial}{\partial x^{*}} \\
\frac{\partial}{\partial y^{*}}
\end{array}\right]
$$

and $\vec{n}^{*}$ the scaled normal vector of the boundary.

The other equations do not contain a spatial variable and therefore do not need to be nondimensionalized. Other special settings concerning the mesh e.g. mesh refinements for regions of high gradients like in the vicinity of the grain boundary or near the iron in-diffusion boundary are described in the appendix in section 7. 


\subsection{Calculation of diffusion length and apparent concentration}

For the comparison with experimental LBIC measurements, the minority carrier lifetime is calculated from the interstitial iron concentration via the Shockley-Read-Hall electron-hole recombination at deep level defects in silicon as described in section 2.4. In order to account for the recombination activity coming from the grain boundary, a model simulating the LBIC current/ the diffusion length contribution from a grain boundary when illuminated with a light beam was developed by Nitzbon [91]. Figure 14 shows the geometry of this model and enumerates the boundaries described in the following text.

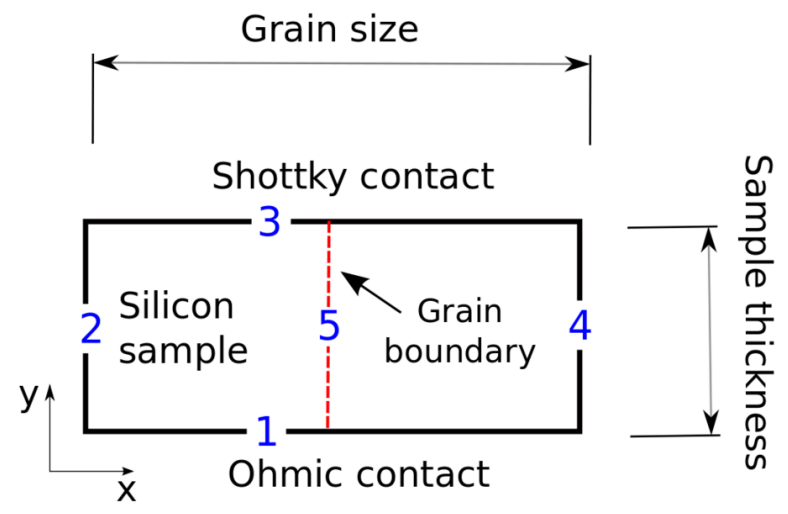

Figure 14: Two dimensional model geometry for the LBIC model.

The input for the model is the calculated diffusion length from the modeled interstitial iron concentration, a recombination velocity of the grain boundary and the properties of the laser beam such as absorption coefficient and beam width. For the values used for the different parameters, please see table 4. For the minority carrier concentration $n$, the minority carrier diffusion equation given in section 2.5 is implemented as two dimensional equation:

$$
-D_{n}\left(\frac{\partial^{2} n(x, y)}{\partial x^{2}}+\frac{\partial^{2} n(x, y)}{\partial y^{2}}\right)+\frac{D_{n}}{L^{2}} n(x, y)=g_{n}(x, y)
$$

with the diffusion coefficient for the minority carriers $D_{n}$, the diffusion length $\mathrm{L}$ and the laser generation rate:

$$
g_{n}(x, y)=\frac{\sqrt{\pi} b \alpha \Phi_{0}}{l_{0}} \cdot \exp \left(-\frac{\left(x-x_{0}\right)^{2}}{b^{2}}\right) \cdot \exp (-\alpha y)
$$

$\Phi_{0}$ is the photon flux of the transmitted laser beam through the silicon surface, $b$ the beam width, $\alpha$ the absorption coefficient of the laser and $l_{0}$ a unit length, which enters the equation when reducing it to two dimensions (please refer to Nitzbon [91] for more details).

The Ohmic contact at the bottom (please see boundary 1 in figure 14 and figure 6 in section 
2.5 for comparison) is described by zero flux conditions (Neumann boundary conditions):

$$
-\vec{n} \cdot\left(-D_{e} \nabla n(x, y)\right)=0
$$

The Schottky contact on the top (boundary 3 in figure 14) is described by Dirichlet boundary conditions:

$$
n(x, y)=0
$$

and the recombination at the grain boundary (boundary 5 in figure 14) is described by Robin boundary conditions:

$$
-\vec{n} \cdot D_{e} \nabla n(x, y)=S_{e f f} \cdot n(x, y)
$$

with the effective recombination velocity at the grain boundary $S_{\text {eff }}$.

For the boundaries 2 and 4 on the sample sides, zero flux conditions are implemented.

The LBIC current $I_{L B I C}$ can be calculated from the minority carrier concentration via integration over boundary 3 :

$$
I_{L B I C}=\left.D_{e} \cdot e \cdot l_{0} \int_{-x_{S i}}^{x_{S i}} \frac{\partial n}{\partial y}\right|_{y=0} d x
$$

with the sample starting at $-x_{S i}$ and ending at $x_{S i}$ and the elementary charge of electrons $e$.

From the LBIC current, the diffusion length $\mathrm{L}$ can be calculated via:

$$
L=\frac{1}{\alpha \cdot\left(\frac{\pi b^{2} e \Phi_{0}}{I_{L B I C}}-1\right)}
$$

For more details on the implementation of the LBIC model please refer to Nitzbon [91]. The table 4 below lists the parameters used in this work:

\begin{tabular}{llll}
\hline \hline Parameter & Value & Description & Reference \\
\hline$<v_{n}>$ & $1.7 \cdot 10^{7} \mathrm{~cm} / \mathrm{s}$ & Mean thermal velocity of minority carriers & {$[7]$} \\
$\sigma_{n}$ & $3 \cdot 10^{-14} \mathrm{~cm}^{2}$ & Capture cross section of the FeB defects for electrons & {$[99]$} \\
$D_{n}$ & $33.5 \mathrm{~cm}^{2} / \mathrm{s}$ & Diffusion coefficient of minority carriers at room temperature & {$[7]$} \\
$k_{L}$ & $1.5 \cdot 10^{13} \mu \mathrm{s} / \mathrm{cm}^{3}$ & Conversion factor for diffusion length & \\
$T_{\text {room }}$ & $25^{\circ} \mathrm{C}$ & Temperature during measurement (room temperature) \\
$\alpha$ & $380 \mathrm{~cm}^{-1}$ & Absorption coefficient of the laser \\
$b$ & $10 \mu m$ & Radius of the laser beam \\
$\lambda_{L}$ & $850 \mathrm{~nm}$ & Laser wavelength \\
$P$ & $5 \cdot 10^{-6} \mathrm{~W}$ & Laser power \\
$\Phi_{0}$ & $P /\left(E_{\text {phot }} \cdot \pi \cdot b^{2}\right)$ & Photon flux with photon energy $E_{\text {phot }}$
\end{tabular}

Table 4: List of parameters for the LBIC model with the used values, references and a description. 


\section{Simulation studies on impurity accumulation at grain bound- aries}

\subsection{Comparison of phosphorus diffusion gettering and aluminum getter- ing in a one dimensional Model}

As described in section 2.2, gettering techniques are used to reduce metal impurity concentrations in solar cells to improve their efficiency. Quantitative simulations are important for further optimization of the gettering techniques. In this section the efficiency and kinetics of two different gettering techniques - phosphorus diffusion gettering (PDG) and aluminum gettering (AlG) - are compared for the example of iron as metal impurity at different gettering temperatures in a one dimensional model. For the details of the model please refer to section 4.1 of this work and references within. Since both, efficiency and kinetics, are influenced by metal impurity precipitates, the metal precipitate density is varied between $N_{p}=0 \mathrm{~cm}^{-3}, 10^{8} \mathrm{~cm}^{-3}$ and $10^{10} \mathrm{~cm}^{-3}$. The initial metal impurity concentration is chosen to be $10^{14} \mathrm{~cm}^{-3}$. The sample thickness is $300 \mu \mathrm{m}$. The simulations are performed for high and low gettering temperature regimes. The regimes are separated by the equilibrium temperature $T_{e q}^{\text {tot }}$, which describes the temperature for which the solubility of the metal impurity corresponds to the total impurity concentration. For an iron concentration of $10^{14} \mathrm{~cm}^{-3}$, the equilibrium concentration is $T_{e q}^{\text {tot }} \approx 940^{\circ} \mathrm{C}$. For AlG the experimentally determined segregation coefficient [39] is used. If not mentioned otherwise, the $\mathrm{Al}: \mathrm{Si}$ gettering layer thickness is $1 \mu m$.

The graphs in figure 15 and 16 show depth profiles for the concentration of interstitial, precipitated and gettered metal atoms for different times after the onset of the aluminum and phosphorus diffusion gettering for a precipitate density of $N_{p}=10^{8} \mathrm{~cm}^{-3}$. The figure 16 is taken from a previous study performed by Seibt et al. [54].

The graphs on the top of figure 15 and 16 show the concentration profiles at the onset of the gettering at low gettering temperature (figures 15(a) and 16(a) and at high gettering temperature (figures 15(b) and 16(b) while the graphs on the bottom show the concentration profiles at later times after the onset of gettering at low (figures 15(c) and 16(c) and high (figures 15(d) and 16(d) gettering temperature. Please note that the aluminum gettering layer starts at $x=0$ outside of the silicon sample.

The figures illustrate that at low gettering temperatures AlG and PDG show similar concentration profiles: the precipitates dissolve slowly because the concentration of the interstitial metal lies above the solubility. After onset of gettering, the interstitial metal concentration is decreased and precipitates dissolve up to the solubility limit - under the assumption that the chemical potential of precipitates is indepdent of precipitate size, i.e. no contributions from surface and strain energy are taken into account in this simulations (see section 4.1 for details). The precipitates serve as source for new interstitial metal impurities. As long as there are precipitates present in the sample the interstitial metal concentration can not decrease below the solubility concentration. Only when all precipitates are dissolved the concentration can decrease to a level determined by the segregation coefficient. 


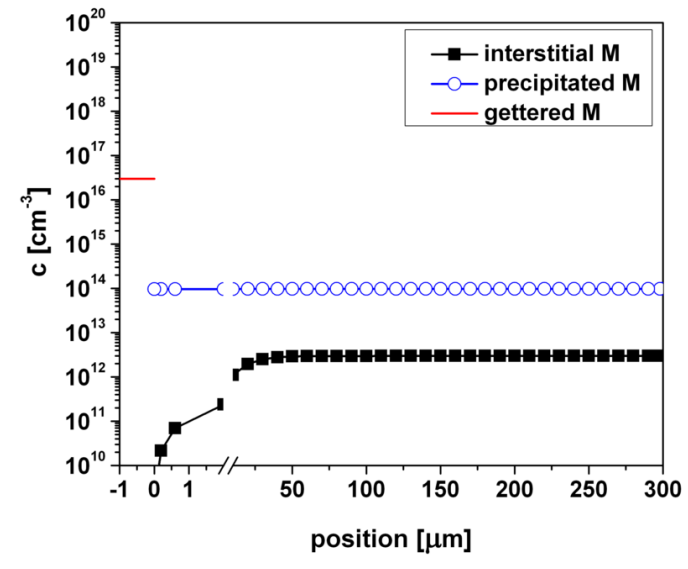

(a) AlG at the onset for low temperature

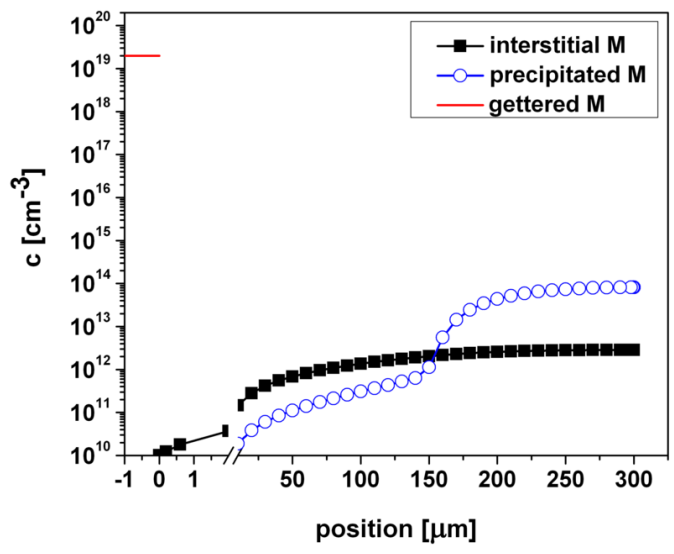

(c) $\mathrm{AlG}$ at later times for low temperature

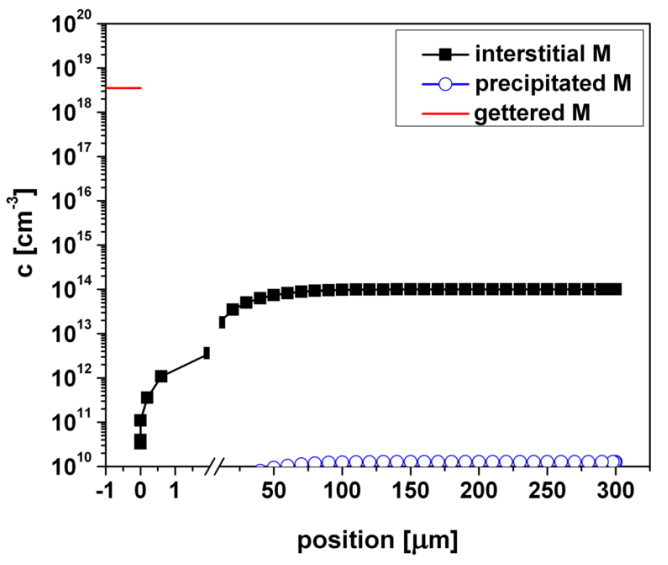

(b) $\mathrm{AlG}$ at the onset for high temperature

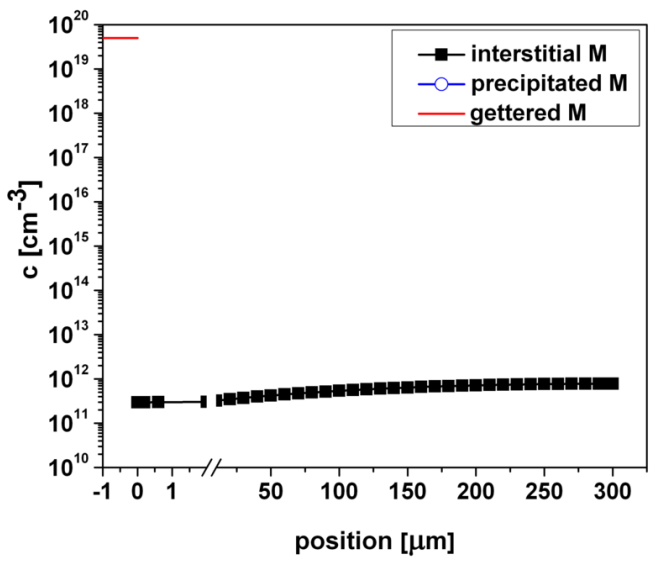

(d) AlG at later times for high temperature

Figure 15: Concentration depth profiles for the interstitial, the precipitated and gettered impurity concentration during AlG for low gettering temperature $[15(\mathrm{a}), 15(\mathrm{c})]$ and high gettering temperature $[15(\mathrm{~b}), 15(\mathrm{~d})]$. On top, the profiles only few seconds after the onset of the gettering are presented, while on the bottom the concentration profiles after some time after the onset of the $\mathrm{AlG}$ are shown. It can be seen that for high temperature the precipitates dissolve immediately and the concentration of interstitial impurity concentration decreases fast, while for low temperature the precipitates dissolve only slowly and the interstitial impurity concentration can not decrease as fast as for high temperature. 


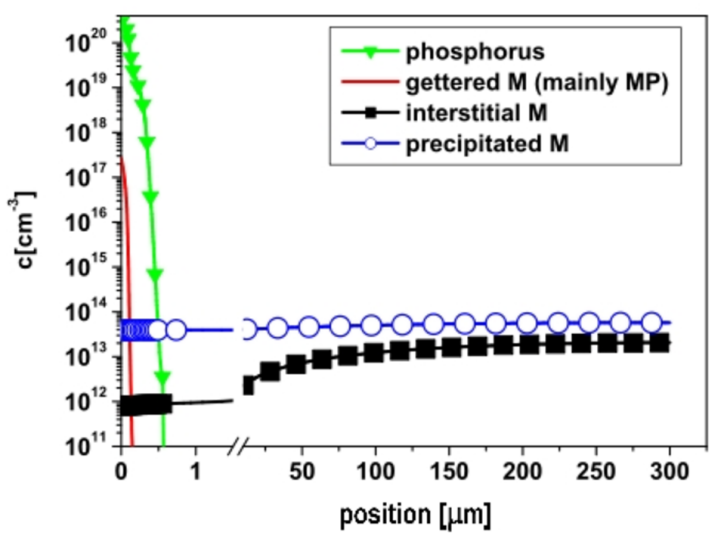

(a) PDG at the onset for low temperature

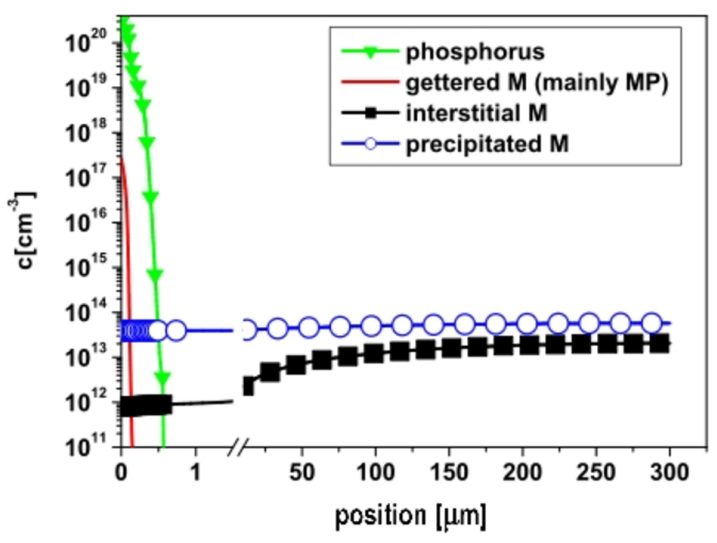

(c) PDG at later times for low temperature

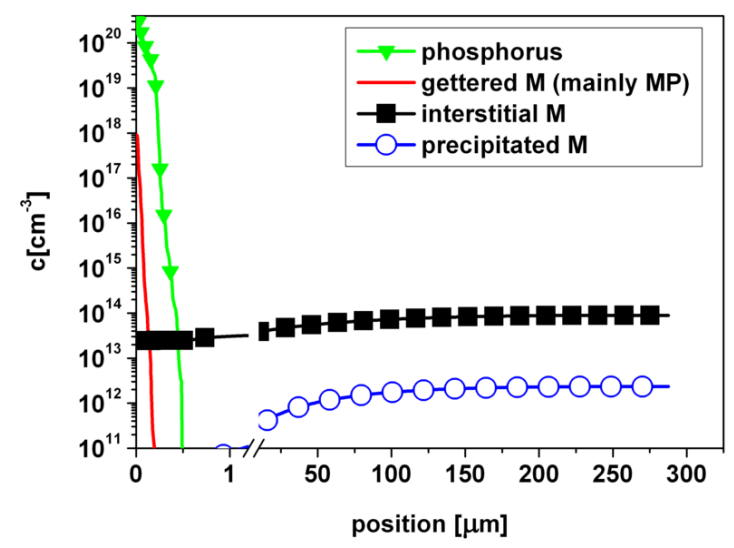

(b) PDG at the onset for high temperature

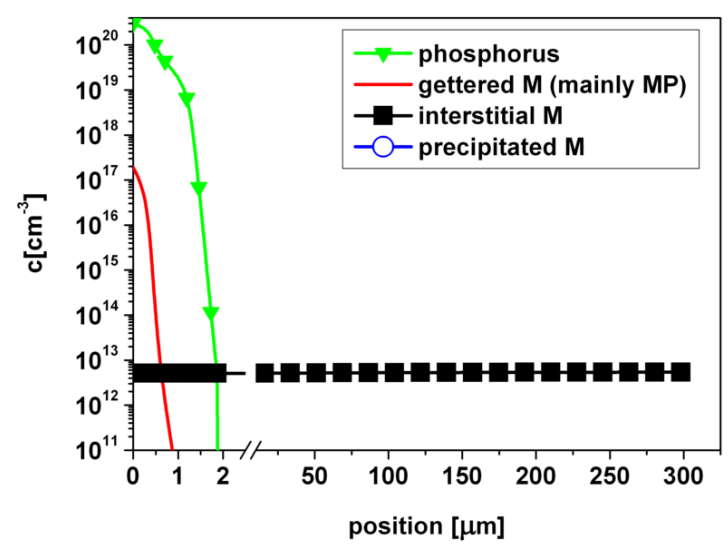

(d) PDG at later times for high temperature

Figure 16: This figure is taken from Seibt et al. [54] to compare PDG with AlG in figure 15. It shows the concentration depth profiles for the interstitial, the precipitated and gettered impurity concentration and the phosphorus concentration during PDG for low gettering temperature 16(a) 16(c) and high gettering temperature $[16(\mathrm{~b}), 16(\mathrm{~d})]$. On the top, the profiles only few seconds after the onset of the PDG are presented, while on the bottom the concentration profiles after some time after the onset of the PDG are shown. It can be seen that the precipitates dissolve faster and the interstitial impurity concentration decreases faster for high gettering temperature. 
For high gettering temperatures (see figures 15(b), 15(d) and 16(b), 16(d)) the precipitates can dissolve almost instantaneously after the onset of gettering and the interstitial metal concentration sinks below the solubility level.

In order to illustrate the differences of PDG and $\mathrm{AlG}$, it is determined which gettering time $t_{g}$ is necessary to decrease the concentration of the metal impurity in a Si-wafer from the initial concentration of $10^{14} \mathrm{~cm}^{-3}$ to a target concentration of $10^{12} \mathrm{~cm}^{-3}$. Figure 17 shows the gettering time $t_{g}$ versus gettering temperature $T_{G}$ for AlG in figure 17(a) and PDG in figure 17(b). The different curves show the results for three different precipitate densities. The triangles with a density of $N_{p}=10^{8} \mathrm{~cm}^{-3}$ correspond to the concentration depth profiles in figures 15 and 16

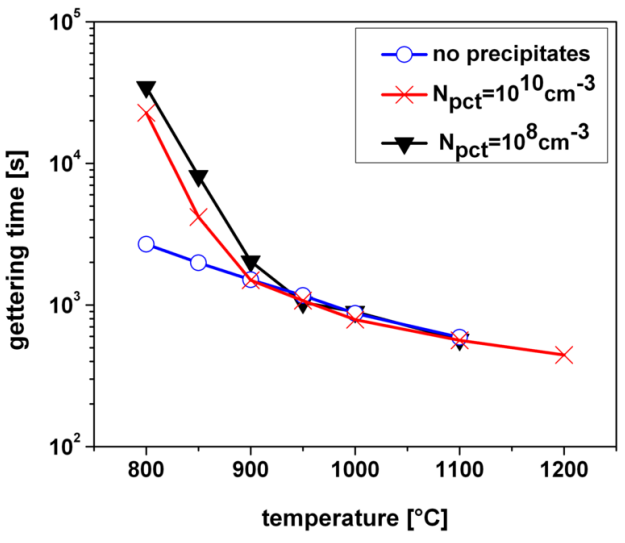

(a) Gettering time for $\mathrm{AlG}$

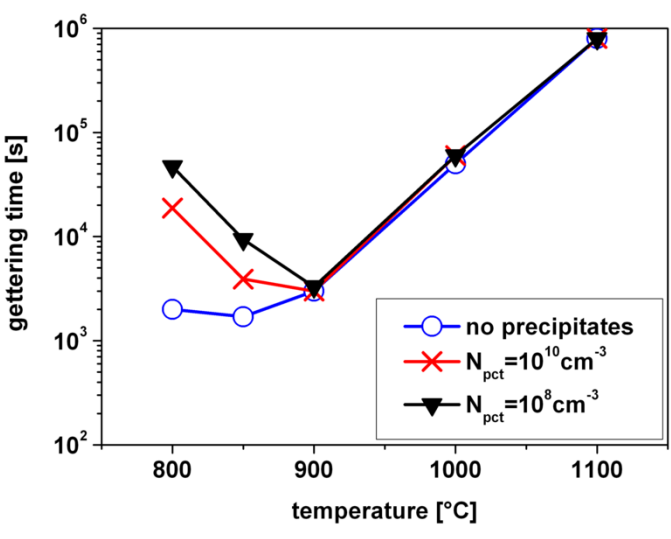

(b) Gettering time for PDG

Figure 17: Gettering time $t_{g}$, which is needed to decrease the concentration of interstitial impurities from an initial concentration of $10^{14} \mathrm{~cm}^{-3}$ to a target concentration of $10^{12} \mathrm{~cm}^{-3}$, versus gettering temperature for AlG and PDG. The figure 17(b) for PDG is taken from Seibt et al. [54]. Shown are three different precipitate densities of $N_{p}=0 \mathrm{~cm}^{-3}, N_{p}=10^{8} \mathrm{~cm}^{-3}$ and $N_{p}=10^{10} \mathrm{~cm}^{-3}$. For PDG the gettering time decreases with increasing temperature for $T_{G}<T_{e q}^{\text {tot }}$, while for $T_{G}>T_{e q}^{t o t}$ the gettering time increases with increasing temperature. This stands in contrast to AlG, for which the gettering time further decreases with increasing temperature in the temperature regime $T_{G}>T_{e q}^{\text {tot }}$.

For the low temperature regime, i.e. a gettering temperature below the equilibrium temperature $T_{G}<T_{e q}^{t o t}$, the gettering time $t_{g}$ decreases with increasing gettering temperature $T_{G}$ for both, PDG and AlG, and the density of precipitates influences the gettering time significantly.

Kinetics in this regime are limited by the dissolution of metal impurity precipitates, which depends on the precipitate size, i.e. on the precipitate density. This result is in agreement with the conclusions of other simulations studies and experimental results [14, 100, 9].

For the high temperature regime, i.e. a gettering temperature above the equilibrium temperature $T_{G}>T_{e q}^{\text {tot }}$, the gettering time $t_{g}$ shows different behavior for PDG in comparison to AlG: 
while for AlG the gettering time $t_{g}$ further decreases with increasing gettering temperature, for PDG the gettering time $t_{g}$ increases again for increasing gettering temperature. Both figures show that the influence of precipitate density is significant neither for PDG nor for AlG in this regime. Since the dissolution of precipitates happens almost instantaneously in this high temperature regime, it does not limit the gettering kinetics as shown experimentally by Sattler [58] for AlG of cobalt. Therefore, the limiting factor for $\mathrm{AlG}$ kinetics in this regime is the increasing diffusion coefficient of metal impurity atoms with increasing gettering temperature. For PDG, kinetics are limited by the in-diffusion of phosphorus and the slower establishment of the gettering layer. The exponentially decreasing segregation coefficient with increasing temperature becomes relevant and dominates over the increasing diffusion coefficient of the metal impurities in the high temperature regime. A further gettering success for PDG is only possible by increasing the segregation coefficient via an increasing gettering layer thickness.

For AlG with a sufficiently thick Al:Si liquid, the segregation coefficient is not limiting because the aluminum gettering layer forms already during heating to the gettering temperature as has been deduced by Sattler [58] from observations of AlG of cobalt.

In order to investigate down to which layer thickness the influence on gettering is insignificant, a variation of the Al:Si liquid thickness with thicknesses of $0.1 \mu \mathrm{m}, 1 \mu \mathrm{m}$ and $10 \mu \mathrm{m}$ was performed as shown in figure 18 . For a gettering layer thickness $\leq 0.1 \mu \mathrm{m}$ above approximately $950^{\circ} \mathrm{C}$ the target concentration can not be reached while for a thickness $\geq 1 \mu \mathrm{m}$ the layer thickness does not influence the gettering time even for high temperatures of $1200^{\circ} \mathrm{C}$. However, it has to be kept in mind that the segregation coefficient influences the gettering efficiency and that the concentration of the interstitial metal can only be decreased to a level which corresponds to the segregation coefficient according to the thickness of the Al layer. Due to the decreasing segregation coefficient with increasing temperature and a decreasing diffusion coefficient with decreasing temperature, it exists an optimum temperature for gettering as stated by e.g. McHugo et al. [8] and Cañizo and Luque [29].

The conclusion of this section is that for a sufficiently thick gettering layer, AlG is only limited by thermodynamic conditions and has the advantage of a decreasing gettering time for increasing temperatures, while for PDG the conflictive effects of decreasing segregation coefficient with increasing temperature and decreasing mobility for decreasing temperature affect the gettering time, leaving only an optimum temperature range around the equilibrium temperature for which the gettering time is low. Even though AlG is not were efficient in practical solar cell processing schemes due to the short firing step [16, 55], its advantages of a comparatively simple underlying physics and the gettering layer outside the sample make it a good technique for fundamental research. Therefore, this work focuses on AlG for further simulation studies to investigate the redistribution of impurities accumulated at grain boundaries into the grains during gettering processes. AlG is implemented into a two dimensional model to include a grain boundary as additional site for impurity accumulation, as already the one dimensional study in this section shows that precipitates influence the gettering kinetics significantly, especially for low temperatures. It is expected that not only evenly spread precipitates in the grain, but also precipitates at the grain boundary act as sinks and sources for impurity accumulation. 


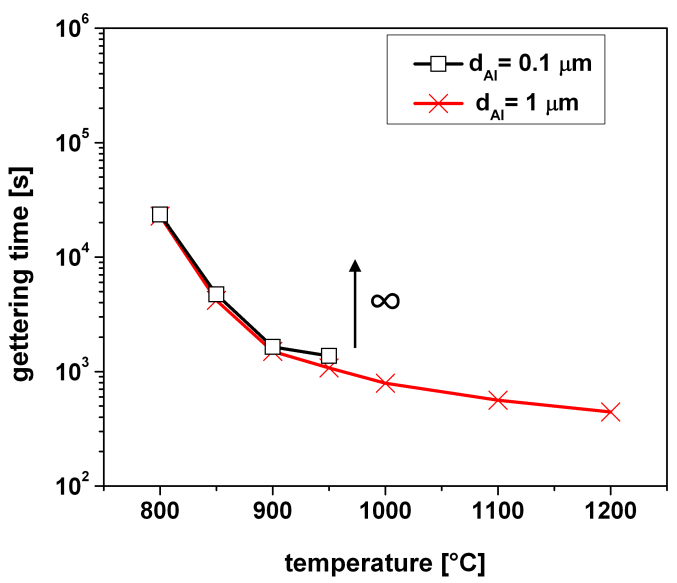

Figure 18: Variation of layer thickness for aluminum gettering. Shown is the gettering time $t_{g}$ which is needed to decrease the concentration of interstitial impurities from an initial concentration of $10^{14} \mathrm{~cm}^{-3}$ to a target concentration of $10^{12} \mathrm{~cm}^{-3}$ versus gettering temperature for a precipitate density of $N_{p}=10^{8} \mathrm{~cm}^{-3}$. It can be seen that for a sufficiently thick gettering layer the thickness does not influence the gettering time, while for a critical thickness the target concentration can not be reached anymore. The critical thickness in this example amounts $\leq 0.1 \mu \mathrm{m}$ above approximately $950^{\circ} \mathrm{C}$. 


\subsection{Aluminum gettering with grid fingers or a grain boundary in a two dimensional model}

In this section simulations of aluminum gettering with a continuous Al:Si liquid, a spatially restricted Al:Si liquid in the geometry of grid fingers (which are used in solar cell industry for alternative backside contact to reduce shading) and aluminum gettering in presence of a grain boundary are compared in order to consider actual conditions of solar cell processing. The one dimensional model for $\mathrm{AlG}$ is expanded to two dimensions because the above mentioned geometries require two dimensional modeling. For details of the model please refer to section 4 of this work. The geometry for the simulation of grid fingers as aluminum gettering layer is shown in figure 19. Modeled is the x-y-plane. The geometry for the simulation of AlG in the presence of a grain boundary is shown in figure 13 in section 4 .

For all geometries in this section, a sample thickness of $300 \mathrm{um}$, a sample size of $4.4 \mathrm{~mm}$ and an Al:Si liquid thickness/ grid finger thickness of $20 \mu \mathrm{m}$ is chosen. The grid fingers have a width of $0.1 \mathrm{~mm}$ and the space between the fingers is $2.1 \mathrm{~mm}$. A metal impurity with the properties of iron is used with a total concentration of $5 \cdot 10^{13} \mathrm{~cm}^{-3}$. The precipitate density is $N_{p}=1 \cdot 10^{8} \mathrm{~cm}^{-3}$ in the grain and $N_{g b}=1 \cdot 10^{7} \mathrm{~cm}^{-2}$ at the grain boundary. The gettering temperature is held constant at $900^{\circ} \mathrm{C}$. For the $\mathrm{AlG}$ with a continuous gettering layer and the grid finger gettering, it is assumed that the samples are in its equilibrium state at the onset of the AlG simulations, i.e. the initial interstitial impurity concentration equals the solubility of iron in silicon at $900^{\circ} \mathrm{C}$, namely $4.26 \cdot 10^{13} \mathrm{~cm}^{-3}$. The remaining impurities are precipitated. For the AlG simulation in the presence of a grain boundary the remaining impurities are distributed between impurities precipitated at the grain boundary and in the grain. In order to determine the amount precipitated at the grain boundary and the amount precipitated in the grain, a simulation is done at $900^{\circ} \mathrm{C}$ until an equilibrium state is reached, i.e. the interstitial iron concentration in the bulk equals the solubility of iron in silicon at $900^{\circ} \mathrm{C}$ and there is no net flux from/ to the precipitates at the grain boundary or the precipitates in the grain. The resulting concentrations are used as initial concentrations.

The final concentrations which can be reached according to the segregation coefficient differ for the simulations with a continuous gettering layer and with grid fingers as gettering layer. This can be deduced from simple calculations. According to the conservation of mass, the following

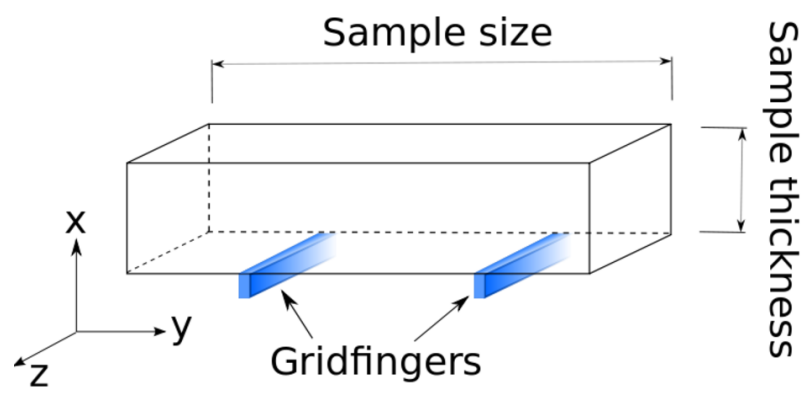

Figure 19: Geometry for AlG simulations with grid fingers. 
equation has to be valid:

$$
w_{S i} \cdot d_{S i} \cdot C_{S i}^{i n i t}=w_{S i} \cdot d_{S i} \cdot C_{S i}^{f i n a l}+w_{A l} \cdot d_{A l} \cdot S_{A l}^{*} \cdot C_{S i}^{\text {final }}
$$

with $w_{S i}$ and $d_{S i}$ being the sample size and thickness, $w_{A l}$ and $d_{A l}$ being the size and thickness of the gettering layer, $C_{S i}^{\text {init }}$ being the initial iron concentration in the silicon and $C_{S i}^{\text {final }}$ being the final concentration in the silicon according to the segregation coefficient $S_{A l}^{*}$ (see equation 4 in section 2.2.1). While for the gettering with grid fingers $w_{A l}$ differs from $w_{S i}$, for the continuous gettering layer it is $w_{S i}=w_{A l}$. The following remaining iron concentrations are derived when using the value for $S_{A l}^{*}$ measured by Abdelbarey et al. [39] and the geometry and gettering temperature used in this simulations for the continuous gettering layer:

$$
C_{S i}^{\text {final }}=1.15 \cdot 10^{9} \mathrm{~cm}^{-3}
$$

and for the grid fingers:

$$
C_{S i}^{\text {final }}=2.56 \cdot 10^{10} \mathrm{~cm}^{-3}
$$

The level of finally remaining interstitial impurity concentration determined by the segregation coefficient is one order of magnitude higher for gettering with grid fingers than for gettering with the continuous gettering layer. 


\subsubsection{Homogeneous aluminum gettering for comparison}

Figure 20 shows two dimensional maps of the logarithmic interstitial impurity concentration (left) and the precipitated impurity concentration (right) at different times after the onset of simple aluminum gettering. The depth profiles for $0 s$ in figure 20(a) and $100 s$ in figure 20(c) show that precipitates can only dissolve when the interstitial impurity concentration decreases below the solubility limit. The depth profiles are comparable to the depth profiles of the one dimensional AlG simulations in the low temperature regime as shown in figure 15 in section 5.1. The only difference of the two simulations is that in the two-dimensional simulation in this section the total initial iron concentration is $5 \cdot 10^{13} \mathrm{~cm}^{-3}$ instead of $1 \cdot 10^{14} \mathrm{~cm}^{-3}$ as in the one-dimensional simulation in section 5.1. The profiles for $1000 s$ in figure 20(e) and 3000s in figure 20(g) illustrate that first all precipitates have to be dissolved before the interstitial impurity concentration can decrease to a level determined by the segregation coefficient: the precipitated impurity concentration reaches $1000 s$ after the onset of $\mathrm{AlG}$ a constant value in the whole sample which equals the minimum concentration allowed in the model (see chapter 4.2 .3 for more details), i.e. all precipitates have dissolved at this time as can be seen in figure 20(f). The interstitial impurity concentration still shows a gradient $1000 s$ after the onset of AlG (figure 20(e)). However, $3000 s$ after the onset of AlG (figure 20(g)), the interstitial impurity concentration has decreased four orders of magnitude and has reached a constant value in the whole sample of approximately $1 \cdot 10^{9} \mathrm{~cm}^{-3}$. This value agrees with the value in equation 64 for the remaining interstitial iron concentration calculated from equation 63 . 


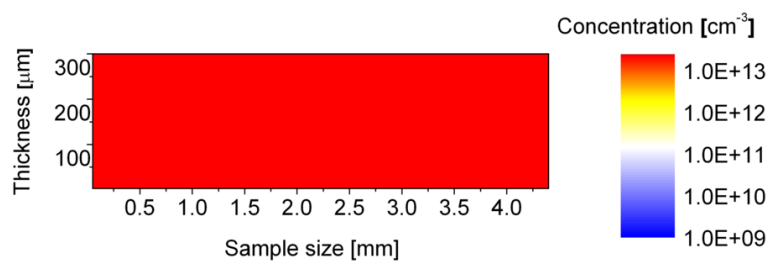

(a) Interstitial metal after $0 \mathrm{~s}$

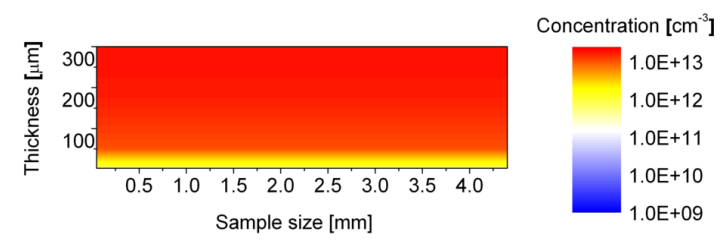

(c) Interstitial metal after $100 \mathrm{~s}$

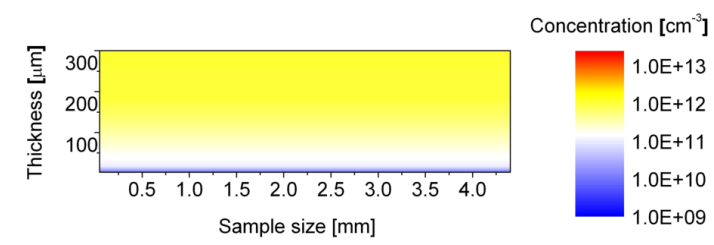

(e) Interstitial metal after $1000 \mathrm{~s}$

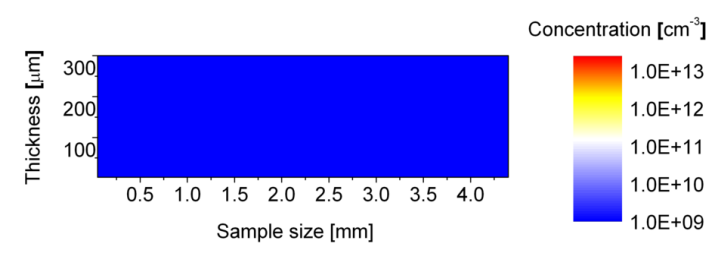

(g) Interstitial metal after 3000s

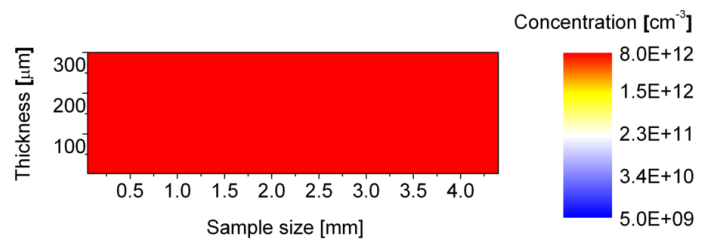

(b) Precipitated metal after $0 \mathrm{~s}$

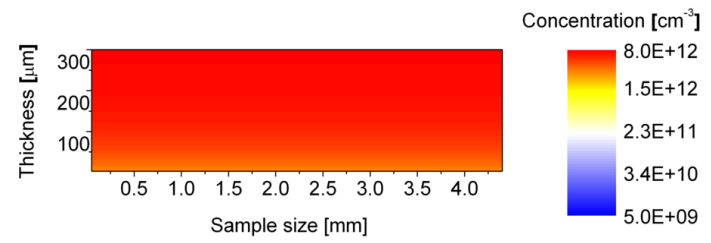

(d) Precipitated metal after $100 \mathrm{~s}$

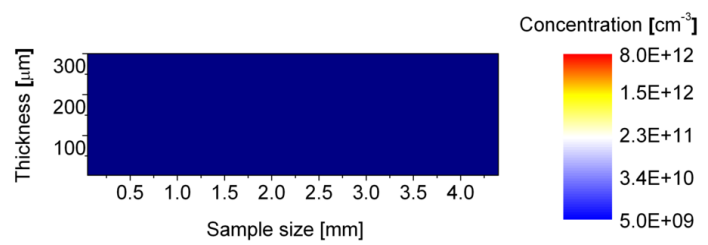

(f) Precipitated metal after $1000 \mathrm{~s}$

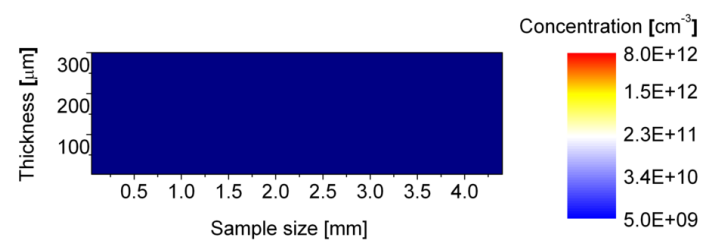

(h) Precipitated metal after $3000 \mathrm{~s}$

Figure 20: $2 \mathrm{~d}$ map of the interstitial impurity concentration (left) and the precipitated impurity concentration (right) $0 s, 100 s, 1000 s$, and 3000s after the onset of $\mathrm{AlG}$. The $\mathrm{Al}$ layer is positioned at the bottom of the sample. The figure shows that the interstitial impurity concentration can only decrease after the precipitates are dissolved. 3000s after the onset of AlG the interstitial iron concentration has reached the value calculated according to the segregation coefficient (equation 64). 


\subsubsection{Aluminum gettering with grid fingers}

Figure 21 shows the logarithmic interstitial (left) and precipitated (right) impurity concentration for different times after the onset of aluminum gettering at $900^{\circ} \mathrm{C}$ with grid fingers as gettering sites instead of a continuous gettering layer. The times are the same as in figure 20

The figure shows that the effect of gettering is locally restricted to the area close to the grid fingers. Even $3000 s$ after the onset of $\mathrm{AlG}$ (see figure 21(g) , the interstitial impurity concentration is still very high in the order of $1 \cdot 10^{13} \mathrm{~cm}^{-3}$. However, the precipitated impurity concentration $3000 s$ after the onset of AlG (see figure 21(h) has decreased to a constant value in the whole sample and has reached the same order of magnitude as for the continuous $\mathrm{Al}$ :Si liquid. $3000 \mathrm{~s}$ after the onset of $\mathrm{AlG}$, the interstitial iron concentration has not reached the value in equation 65 for the final iron concentration calculated via equation 63 according to the segregation coefficient. From this it can be deduced that the difference of the final concentrations in comparison to the gettering with a continuous gettering layer is due to kinetic reasons. 


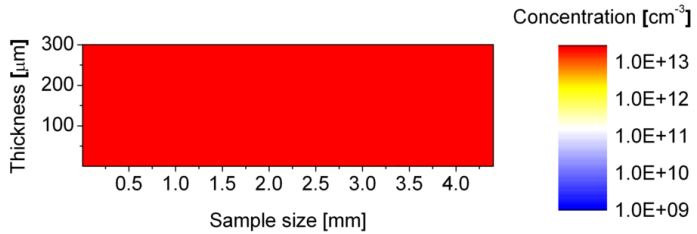

(a) Interstitial metal after $0 \mathrm{~s}$

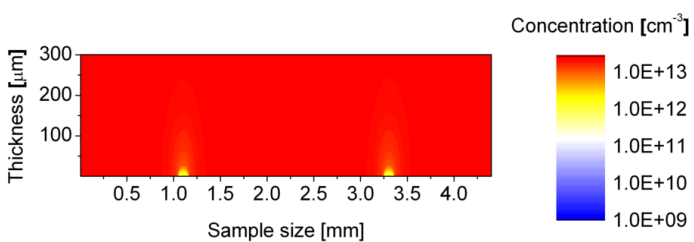

(c) Interstitial metal after $100 \mathrm{~s}$

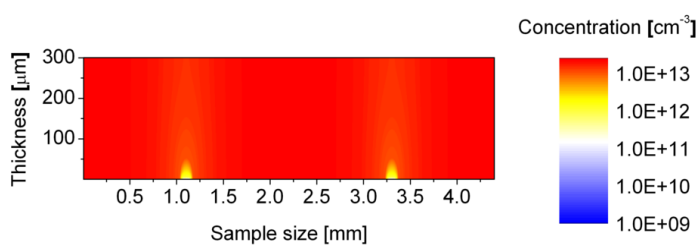

(e) Interstitial metal after $1000 \mathrm{~s}$

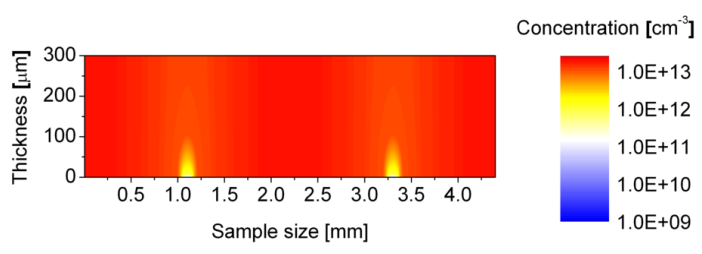

(g) Interstitial metal after $3000 \mathrm{~s}$

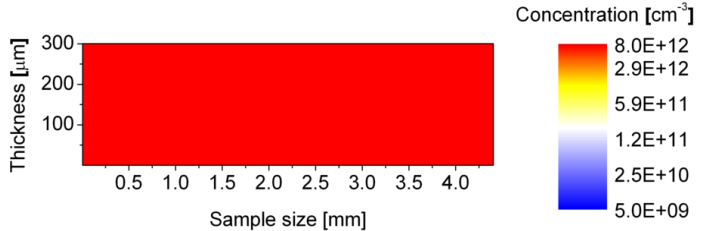

(b) Precipitated metal after $0 \mathrm{~s}$

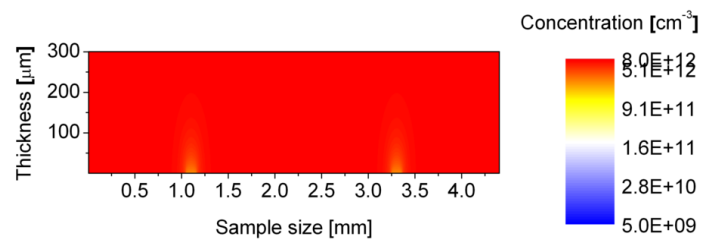

(d) Precipitated metal after $100 \mathrm{~s}$

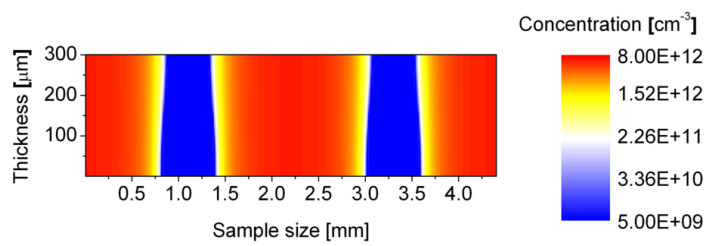

(f) Precipitated metal after $1000 \mathrm{~s}$

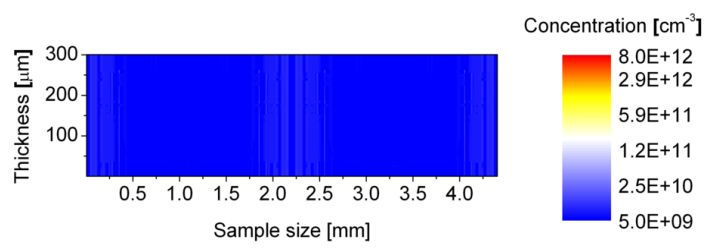

(h) Precipitated metal after $3000 \mathrm{~s}$

Figure 21: $2 \mathrm{~d}$ map of the interstitial impurity concentration (left) and the precipitated impurity concentration (right) $0 s, 100 s, 1000 s$, and $3000 s$ after the onset of $\mathrm{AlG}$ with grid fingers. The $\mathrm{Al}$ grid fingers are positioned at the bottom of the sample. Due to kinetic limitations, the interstitial iron concentration can not decrease to the value calculated according to the segregation coefficient (see equation 65). Interesting is that the concentration of precipitated impurties reaches a value similat to $\mathrm{AlG}$ with a continuous gettering layer (compare figure 20). 


\subsubsection{Aluminum gettering in presence of a grain boundary}

Figure 22 shows the logarithmic interstitial and precipitated impurity concentration from a simulation of AlG in the presence of a grain boundary. In this simulation it was assumed that precipitation at the grain boundary is the only mechanism of accumulation of impurities at the grain boundary. The influence of segregation will be investigated separately in section 5.5. For details how the precipitation at the grain boundary is implemented, please refer to section 4.2.4. While the interstitial impurity concentration at onset of gettering has the same value as for the two previous simulations, the precipitated impurity concentration is lower in comparison to the simulations of AlG without a grain boundary (see figures 20 and 21) because the concentration of the precipitated impurity is distributed among the precipitated component in the grains and the precipitated component at the grain boundary. In contrast, in the simulations without a grain boundary only the precipitated component in the grain is present.

The time evolution of the maps of the interstitial impurity concentration shows an enhanced concentration in the vicinity of the grain boundary. This indicates that the grain boundary acts as local source for interstitial impurity during gettering, as the interstitial impurity concentration in the grains decreases below the solubility limit. This out-diffusion of metal impurities from precipitates located at grain boundaries after gettering processes has been observed in experimental measurements by several authors [5, 24, 79] and has been used to fit experimental results by simulations [25].

For long times all precipitates at the grain boundaries are dissolved and the interstitial impurity concentration sinks fast to a similar level of gettering without a grain boundary. However, the contamination of the grain with the impurities from the grain boundary is still visible, even for long times of $3000 \mathrm{~s}$ after the onset of the AlG (see figure $22(\mathrm{~g})$ ). The map of grain precipitates $100 s$ after the onset of AlG (figure 22(d)) also shows an increased concentration in the vicinity of the grain boundary, reflecting that the interstitial impurity concentration can not decrease in this region as fast due to the re-contamination with impurities from the grain boundary. 


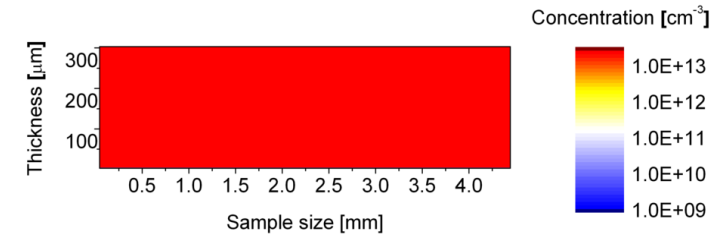

(a) Interstitial metal after $0 \mathrm{~s}$

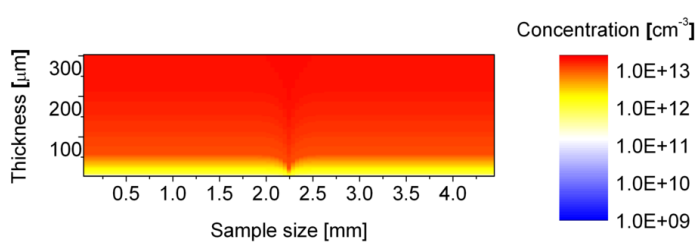

(c) Interstitial metal after $100 \mathrm{~s}$

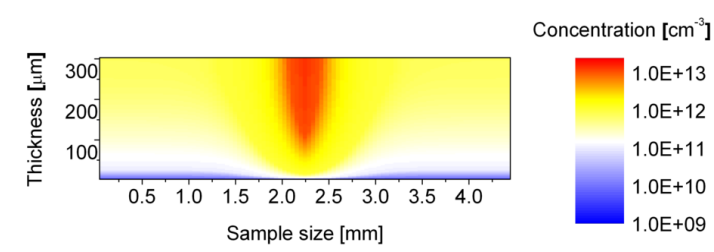

(e) Interstitial metal after $1000 \mathrm{~s}$

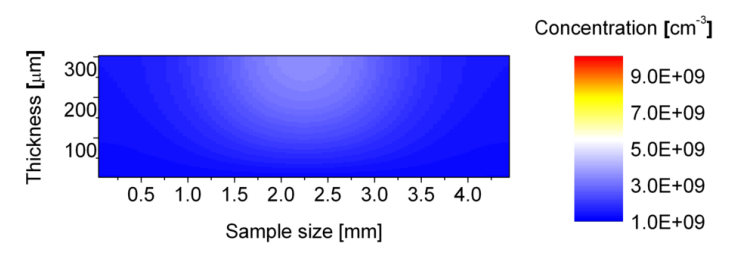

(g) Interstitial metal after $3000 \mathrm{~s}$

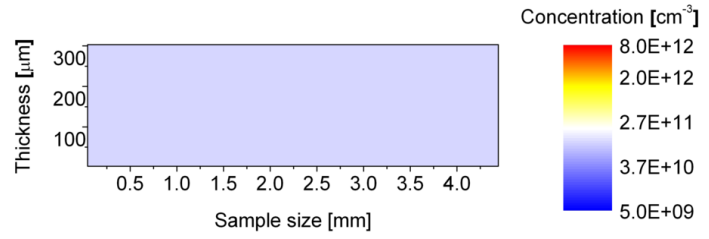

(b) Precipitated metal after $0 \mathrm{~s}$

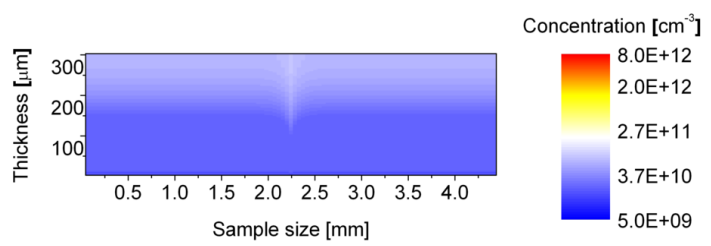

(d) Precipitated metal after $100 \mathrm{~s}$

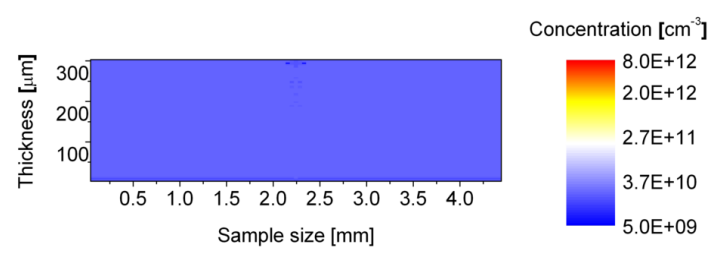

(f) Precipitated metal after $1000 \mathrm{~s}$

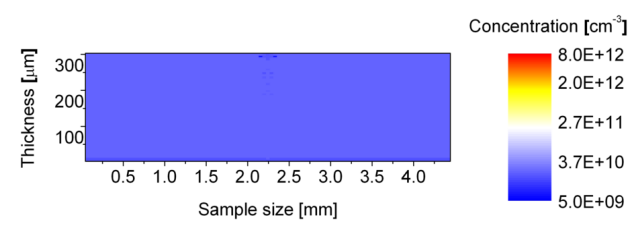

(h) Precipitated metal after $3000 \mathrm{~s}$

Figure 22: $2 \mathrm{~d}$ map of the interstitial impurity concentration (left) and the precipitated impurity concentration (right) $0 s, 100 s, 1000 s$, and 3000s after the onset of AlG in presence of a grain boundary. The Al layer is positioned at the bottom of the sample, the grain boundary is perpendicular to the $\mathrm{Al}$ layer and located in the middle of the sample at the position $x=2.2$. It is illustrated that the grain boundary acts as source for impurities and therefore influences the kinetics during gettering. 


\subsubsection{Comparison of the integral concentrations for the different gettering scenarios}

Figure 23 shows the amount of impurities remaining as interstitial $23(\mathrm{a})]$, precipitated $(23(\mathrm{~b})]$ and gettered (23(c)] metal in the sample for continuous aluminum gettering (black rectangles), gettering with grid fingers (blue triangles) and gettering in the presence of a grain boundary (red circles).

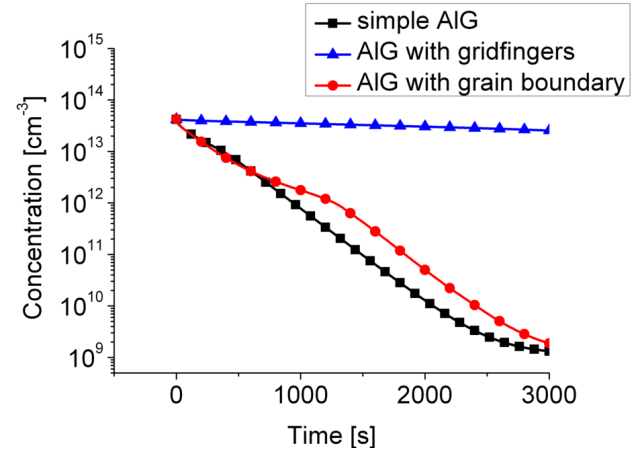

(a) Interstitial metal

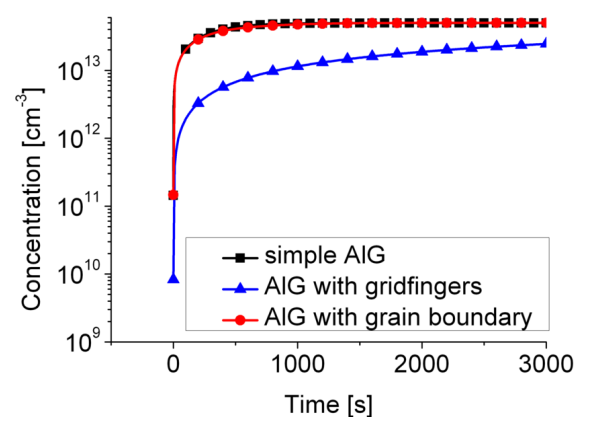

(c) Gettered metal

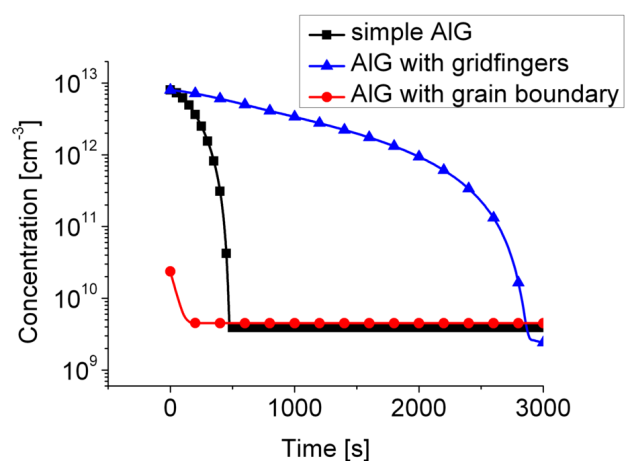

(b) Precipitated metal

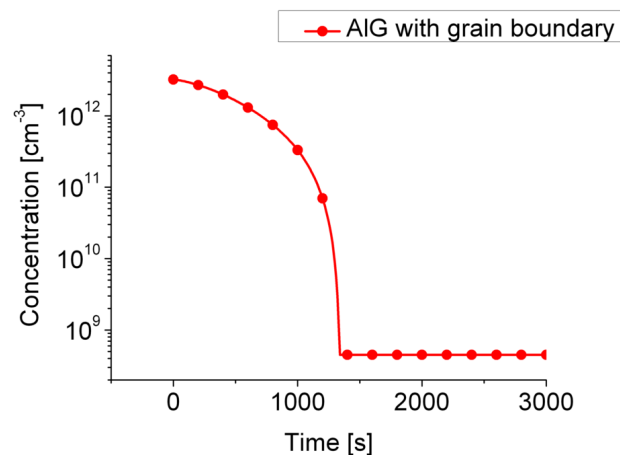

(d) Precipitated metal at the grain boundary

Figure 23: Interstitial, precipitated and gettered amount of the metal impurity concentration versus time for $\mathrm{AlG}, \mathrm{AlG}$ with grid fingers and $\mathrm{AlG}$ with grain boundary in logarithmic scale. Figures 23(a) and 23(c) show that during grid finger gettering singnificant less metal is gettered than for getetring with a continuous gettering layer. It also illustrates that the grain boundary influences the gettering kinetics. A comparison of figure 23(b) and 23(d) shows that the precipitates in the grain dissolve faster than the precipitates at the grain boundary.

The comparison of gettering with grid fingers and gettering with a continuous gettering layer shows that grid finger gettering has an effect, but is much less effective than gettering with a continuous gettering layer. While for the continuous gettering layer the final concentration according to the segregation coefficient is reached $3000 s$ after the onset of the gettering, it takes a much longer time for the gettering with grid fingers until the equilibrium state according to the segregation coefficient is reached. Also the final remaining iron concentration according to the segregation coefficient is one order of magnitude higher than for the gettering with the continu- 
ous gettering layer. However, interesting is that during grid finger gettering the grain precipitates can dissolve until no precipitates are remaining $3000 s$ after the onset of gettering (see figure 23(b)).

The comparison of the gettering with the continuous gettering layer with and without a grain boundary shows that for medium times more interstitial metal remains in the sample (figure 23(a)). The difference in the amount of remaining interstitial impurities can be explained by looking at the concentration of impurities precipitated at the grain boundary as shown in figure 23(d); while 500s after the onset of AlG all grain precipitates have already been dissolved, the precipitates at the grain boundary are still present and serve as source for new interstitial impurities. A comparison of figure 22 and 20 shows that this effect is spatially restricted to the regions near the grain boundary (here this region is approximately $1 \mathrm{~mm}$ wide).

It can be concluded from this section that gettering with grid fingers as gettering layer has some gettering effect, but due to the kinetic limitations long times are needed for effective reduction of the interstitial iron concentration. Furthermore, the final concentration which can be reached according to the segregation coefficient is one order of magnitude higher than for gettering with a continuous gettering layer. Therefore, gettering with grid fingers does not give the desired results for the typically applied processing schemes. However, the simulation of gettering with grid fingers predicts interesting conditions for experiments to investigate the influence of precipitates e.g. on the diffusion length due to the low precipitated iron concentration comparable to gettering with a continuous gettering layer while the interstitial iron concentration is still high.

The section also showed that there is a significant influence of grain boundaries, which serve as source for new impurities during the gettering process, on the gettering efficiency. This is especially true, since in multicrystalline silicon usually the grains are smaller than the grains in this simulation. A good understanding of the interaction of impurities with grain boundaries is needed to optimize gettering in the presence of grain boundaries. The following chapters will show simulation studies in comparison with experiments on the accumulation of impurities at grain boundaries. 


\subsection{Impurity accumulation at grain boundaries}

In this section a simulation study to investigate the influence of temperature treatments on the accumulation of impurities at grain boundaries is presented. For this study the simulations are compared to two different experimental studies from Krenckel [42] measuring LBIC and Liu et al. [35] and Liu and Macdonald [53] measuring PL on grain boundaries of multicrystalline silicon samples from neighbor wafers after different temperature treatments. In the simulations, only precipitation as mechanism of impurity accumulation is taken into account. A review of observations concerning impurity accumulation at grain boundaries in the literature is given in section 2.3, which describes that grain boundaries can act as sink or as source for impurities during temperature treatments.

\subsubsection{Simulations of as-grown wafers for comparison with LBIC measurements}

For the purpose of investigating grain boundaries with LBIC after different temperature treatments, several experiments were performed by Krenckel [42] on block-cast multicrystalline ptype silicon samples intentionally contaminated with iron. The samples were produced in the content of the SolarFocus research cluster (Grant No. 0327650 B). The total iron concentration was measured by NAA (neutron activation analysis) and amounts $5 \cdot 10^{13} \mathrm{~cm}^{-3}$ according to Riepe et al. [87]. The experiments were performed on samples with a size of $10 \times 10 \mathrm{~mm}^{2}$ from the upper third of the block. The original thickness of the samples was $200 \mu m$.

Figure 24 shows temperature curves that were recorded during the experiments and directly used as input for simulations. They were chosen to investigate a variation of temperature (slow $900^{\circ} \mathrm{C}$, slow $950^{\circ} \mathrm{C}$, slow $1025^{\circ} \mathrm{C}$ ) and a variation of cooling rate of $0.05 \mathrm{~K} / \mathrm{s}$ (slow $900^{\circ} \mathrm{C}$ ) and $0.3 \mathrm{~K} / \mathrm{s}$ (fast $900^{\circ} \mathrm{C}$ ). The slow cooling was achieved by leaving the sample in the furnace during cooling, while for the fast cooling the sample was taken out of the furnace and cooled in air. The blue solid line in figure 24 shows a typical temperature curve for cooling after the crystallization process for the used samples. The temperature profile is taken from simulations by Behnken [101] for the SolarFocus research cluster.

Table 5 lists the diffusional ranges $R_{M}$ of iron, i.e. the mean distance the interstitial iron travels during the different cooling processes, calculated by a numerical evaluation based on equation 3 in section 2.1.2. The comparison of the cooling rates $\beta$ and the diffusional ranges $R_{M}$ illustrates that the study covers a wide range of time scales on which diffusion of impurities towards accumulation sites such as grain boundaries can take place.

\begin{tabular}{lll}
\hline \hline Simulation & Cooling rate $\beta\left[\frac{K}{s}\right]$ & Diffusional range $R_{M}[\mathrm{~mm}]$ \\
\hline As grown & 0.018 & 1.25 \\
Fast $900^{\circ} \mathrm{C}$ & 0.3 & 0.16 \\
Slow $900^{\circ} \mathrm{C}$ & 0.05 & 1.03 \\
Slow $950^{\circ} \mathrm{C}, 1025^{\circ} \mathrm{C}$ & 0.05 & 1.15
\end{tabular}

Table 5: Diffusional ranges for the simulations with the temperature curves from figure 24. 


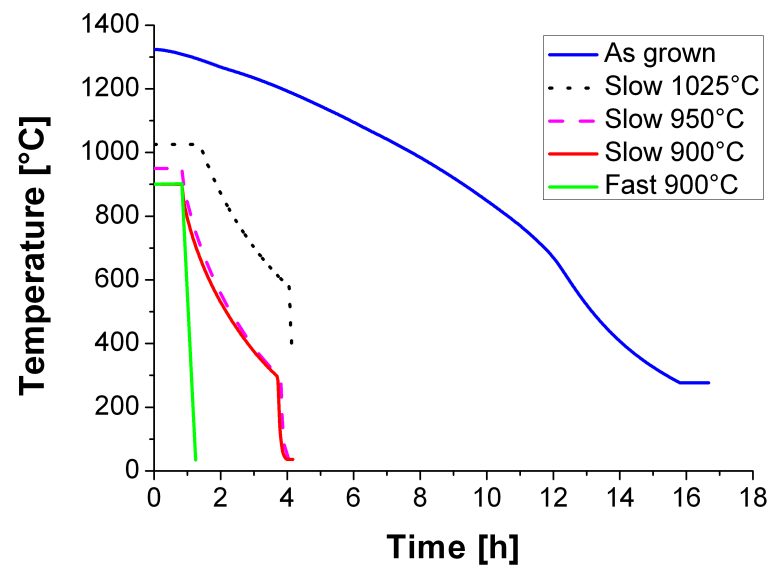

Figure 24: Temperature curves used in this study. The blue line shows simulation results from Behnken [101]. The other curves were recorded during experiments performed by Krenckel [42].

Figure 25 shows two dimensional LBIC maps of two different as-grown samples named 'LBIC 1' and 'LBIC 2' taken from Krenckel [42]. Dark features indicate low currents, i.e a high recombination activity, while bright features indicate high currents, i.e. a low recombination activity. Grain boundaries have a high recombination activity and therefore can be seen as dark features, while there are regions of enhanced LBIC currents in the vicinity of the grain boundaries. These regions of enhanced LBIC currents are interpreted as depletion of impurities near the grain boundaries caused by impurity accumulation at the grain boundary as they have been observed in as-grown wafers by many other authors [24, 5, 27, 78, 25, 68]. In order to compare the experimental measurements with each other, the diffusion length was calculated from the measured LBIC currents for two lasers with different wavelength of $850 \mathrm{~nm}$ and $730 \mathrm{~nm}$. For details on the experiments and the calculation of diffusion length from the LBIC currents please refer to Krenckel [42] and Krenckel et al. [23].

For the simulations of the as-grown sample, i.e. the simulation with the temperature curve for cooling after the crystallization process, a homogeneous iron concentration of $5 \cdot 10^{13} \mathrm{~cm}^{-3}$ was chosen as initial interstitial impurity concentration assuming that at $1300^{\circ} \mathrm{C}$ all iron is dissolved. The density of precipitates was adjusted to $N_{p}=1.5 \cdot 10^{7} \mathrm{~cm}^{-3}$ in the bulk and $N_{g b}=1 \cdot 10^{7} \mathrm{~cm}^{-2}$ on the grain boundary to fit the measured line scans across the grain boundaries shown in figure 25. The thickness of the sample was chosen to $180 \mu \mathrm{m}$ and the grain size to $2 \mathrm{~mm}$, which equals approximately the sizes for the grains at which the line scans were extracted. The grain boundary is located at the position $x=0 \mathrm{~mm}$ and is perpendicular to the sample surface. Fig. 26 shows a two dimensional map of the interstitial iron concentration of the as-grown simulation after approximately $17 \mathrm{~h}$.

The reduced concentration in the vicinity of the grain boundary (position indicated by the black arrow), which will further be called denuded zone, is caused by the accumulation of impurities at the grain boundary during cooling. The denuded zone relates to the depletion regions 


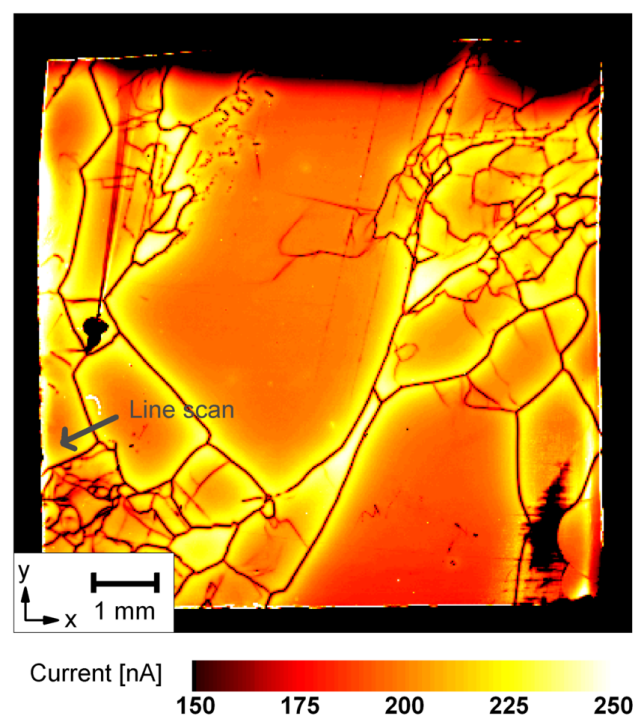

(a) Sample named 'LBIC 1'

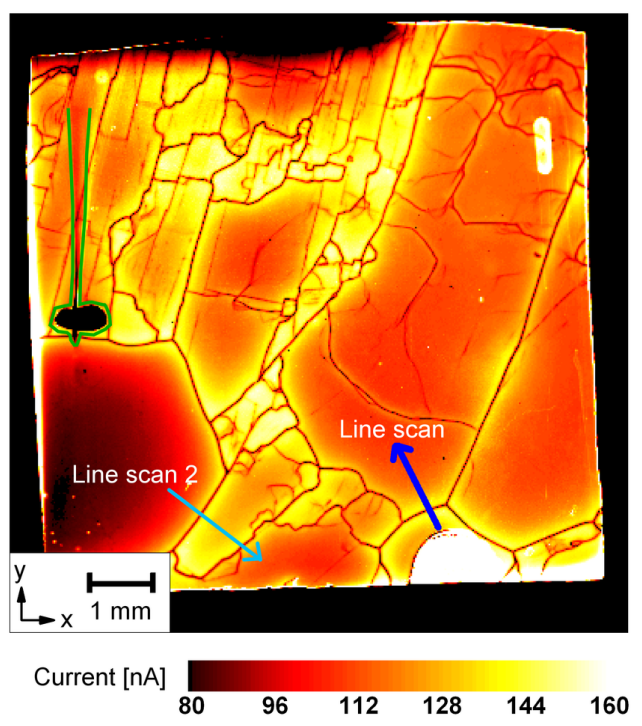

(b) Sample named 'LBIC 2'

Figure 25: LBIC maps for two as-grown samples measured with a laser wavelength of 850 nm. Different grain boundaries, seen as dark lines, separate the sample into grains of different sizes. The arrows mark different line scans shown in the following text. The green mark indicates the front contact. Figures are taken from Krenckel [42].

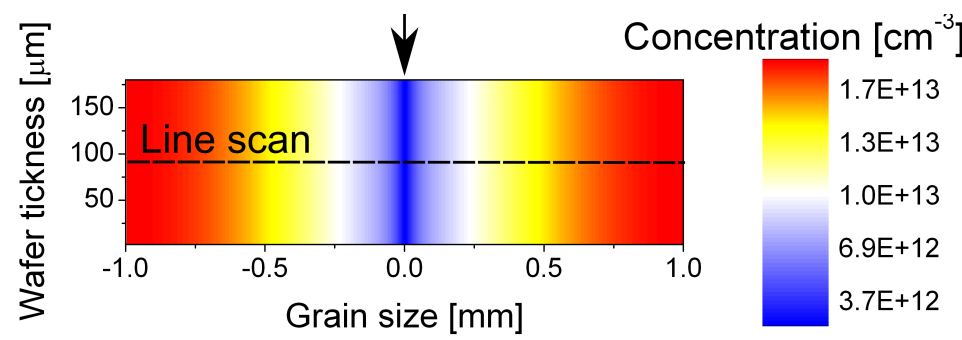

Figure 26: Modeled two dimensional map of the interstitial iron concentration in the presence of a grain boundary at a position $x=0 \mathrm{~mm}$ (indicated with the black arrow) with the as-grown temperature curve after approximately $17 h$. 
seen in figure 25 under the assumption that the LBIC current in this sample is limited by the interstitial iron concentration.

In order to compare simulations directly with the experimental measurements, the diffusion length calculated by Krenckel [42] can be transferred into an 'apparent interstitial iron concentration' as described in section 2.4, assuming that the interstitial iron concentration is the limiting factor for the diffusion length in the sample. Figure 27(a) shows a line scan of the modeled interstitial iron concentration across the grain boundary and a line scan of the measured apparent interstitial iron concentration across the grain boundary. The positions of the line scans are marked by the black line in figure 26 and by the gray arrow in figure 25(a). Figure 27(b) shows a line scan of the apparent interstitial iron concentration across the grain boundary indicated as blue arrow in figure 25(b) and the corresponding modeled line scan. The precipitate densities in the simulation are set to $N_{p}=1 \cdot 10^{6} \mathrm{~cm}^{-3}$ in the bulk and $N_{g b}=4 \cdot 10^{6} \mathrm{~cm}^{-2}$ on the grain boundary in order to fit the measured line scans. Modeled profiles and measured line scans are in close accordance except in the region very close to the grain boundary.

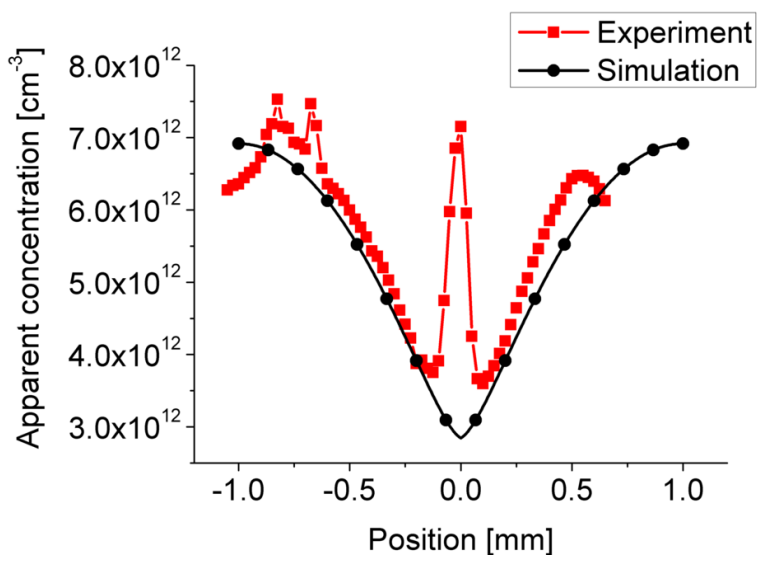

(a) Measured and modeled line scan corresponding to sample LBIC 1

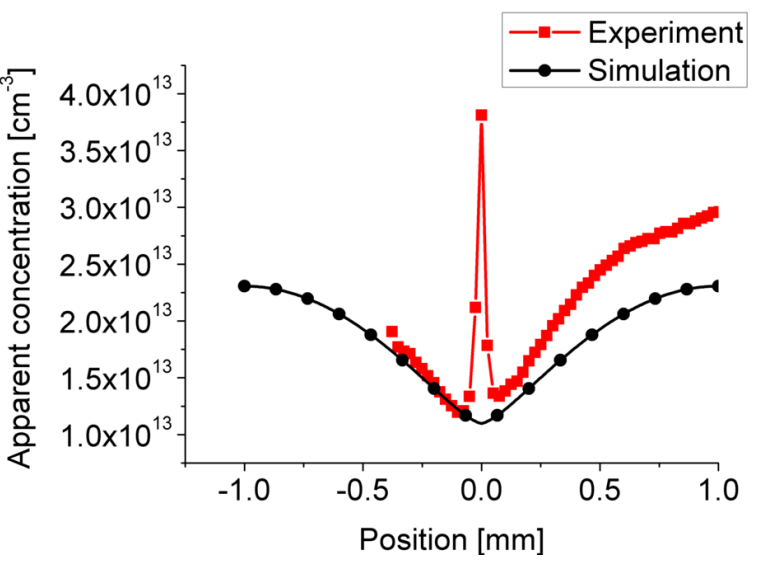

(b) Measured and modeled line scan corresponding to sample LBIC 2

Figure 27: Comparison of a line scan across the grain boundary for the modeled interstitial iron concentration and the calculated apparent interstitial iron concentration from measured LBIC currents by Krenckel et al. [23]. Figure 27(a) shows the line scan on the position in sample LBIC 1 indicated by the dark blue arrow in figure 25(a) and figure 27(b) shows the line scan on the position in sample LBIC 2 indicated by the dark blue arrow in figure 25(b)

The enhanced apparent concentration in the region very close to the grain boundary (for a distance to the grain boundary $<0.2 \mathrm{~mm}$ ) in the line scans measured with LBIC originates from the recombination activity of the grain boundary itself, which is not included in the modeled apparent concentrations. This effect from the grain boundary recombination activity can be modeled with the aid of an LBIC model, which uses the modeled interstitial iron concentration, a recombination velocity of the grain boundary and the conditions of the laser as input as described in section 4.3. Figure 28 shows that modeled and measured apparent interstitial iron concentrations are 
in good accordance for effective recombination velocities of $50 \frac{\mathrm{m}}{\mathrm{s}}$ for figure $28(\mathrm{a})$ and $200 \frac{\mathrm{m}}{\mathrm{s}}$ for figure 28(b). The values used for the laser conditions are given in in section 4 . The good quantitative agreement of the modeled and measured peaks, resulting from the recombination activity of the grain boundary itself, open a new possibility to quantitatively investigate the correlation of the recombination strength of a grain boundary and its dependence on the contamination level of the grain boundary, as it has been observed qualitatively [67]. However, this is beyond the scope of this work.

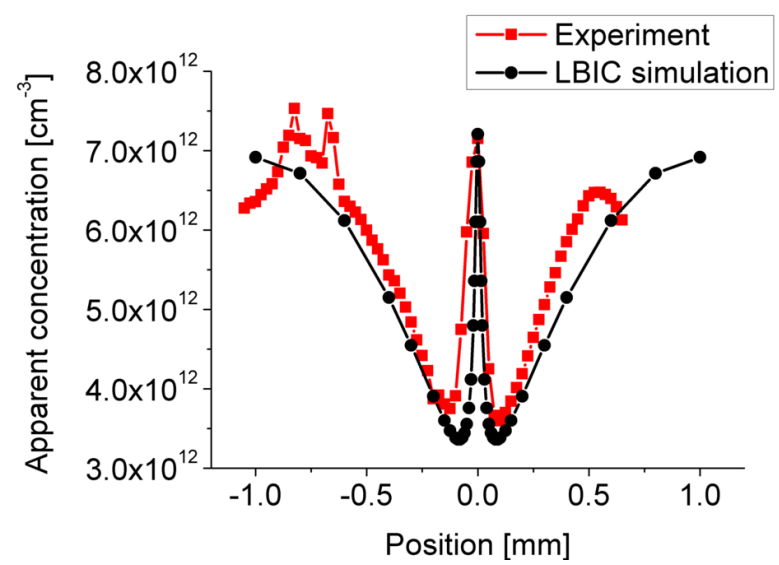

(a) Measured and modeled line scan corresponding to sample LBIC 1

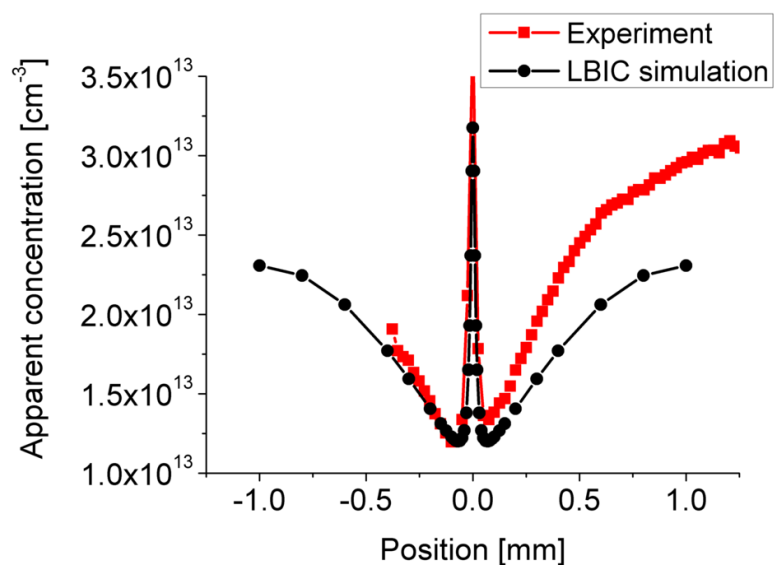

(b) Measured and modeled line scan corresponding to sample LBIC 2

Figure 28: Modeled apparent concentration with the LBIC model in order to include the grain boundary effect seen in measured apparent concentrations. Figure 28(a) corresponds to the line scans shown in figure 27(a), figure 28(b) corresponds to the line scans in figure 27(b)

The good agreement of experiment and simulations shows that the apparent concentrations measured by LBIC can fully be explained by the underlying physics of the model, in which only precipitation as mechanism of impurity accumulation at the grain boundary is taken into account. Therefore, the model can be used to give more insight into the effects of impurity accumulation at grain boundaries on the apparent interstitial iron concentration and can deliver information that can not be achieved with the experiments like, for example, the time evolution of the interstitial iron concentration and the concentration of precipitated impurities in the grain and at the grain boundary during the cooling process as shown in figure 29 .

Figure 29(a) and 29(b) show line scans across the grain boundary of the interstitial iron concentration and the concentration of impurities precipitated in the grains of the as-grown simulation shown in figure 27(b), Figure 29(c) shows the concentration of precipitated iron at the grain boundary versus time. Please note that the precipitated iron concentration at the grain boundary in figure 29(c) is given in $\mathrm{cm}^{-2}$ as it is a two-dimensional boundary.

The time evolution of the interstitial iron concentration in figure 29(a) illustrates, how the denuded zone develops until it reaches the shape that is shown in figure 27(b), approximately $9 \mathrm{~h}$ after the beginning of the cooling the temperature has decreased to a temperature $T=908^{\circ} \mathrm{C}$, which corresponds to the equilibrium temperature $T_{e q}^{i}$ at which the interstitial iron concentration 
of $5 \cdot 10^{13} \mathrm{~cm}^{-3}$ equals the solubility of iron. At this time the precipitates start to grow in the grains (see figure 29(b) and at the grain boundary (see figure 29(c)). The grain precipitation causes a reduction of interstitial iron concentration in the grains and the precipitation at the grain boundary causes the formation of the denuded zone. The time evolution of the concentration of precipitated iron at the grain boundary illustrates that the precipitation takes place only during a short time period of the whole cooling process from the time when $T_{e q}^{i}$ is reached until the mobility of iron has decreased so much that the impurities do not reach the grain boundary anymore.

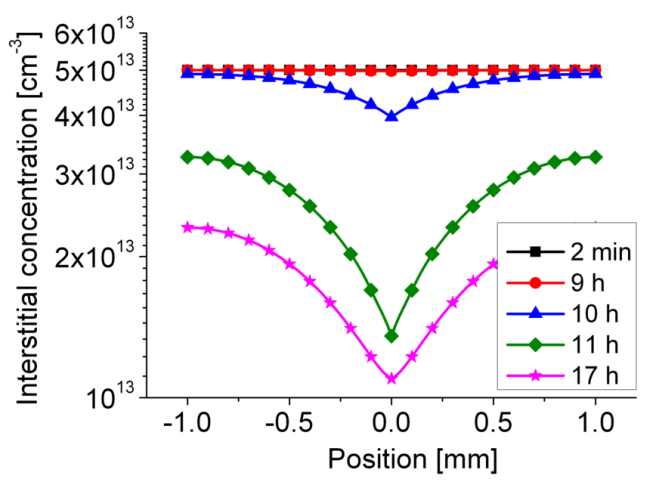

(a) Interstitial iron concentration

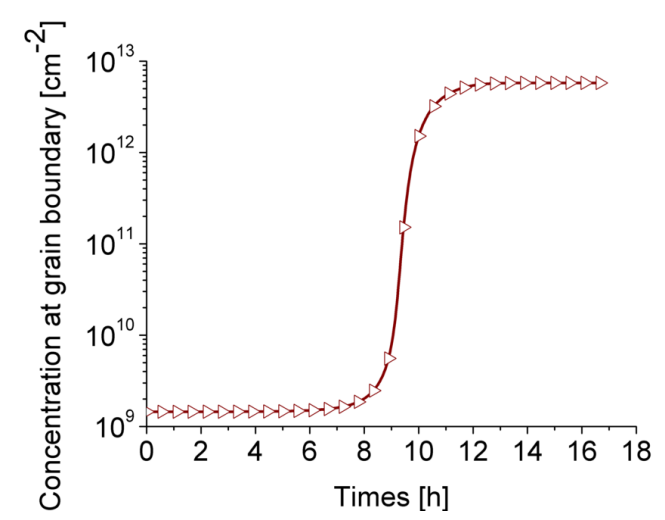

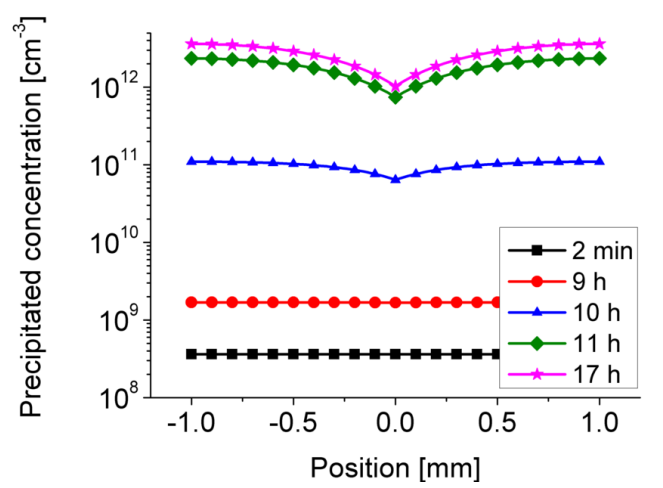

(b) Concentration of precipitated iron in the grain

(c) Concentration of precipitated iron at the grain boundary

Figure 29: Time evolution of the different components of the iron concentrations for the simulation of the as-grown sample LBIC 2 until the state in figure 27(b) is reached. It illustrates how the denuded zone developes. 


\subsubsection{Variation of temperature and cooling rate for comparison with LBIC measurements}

In the subsequent temperature treatments on the as-grown wafers, the variation of temperature was chosen in order to investigate the behavior for an annealing temperature below and above the equilibrium temperature $T_{e q}^{t o t}$, at which the solubility equals to total iron concentration in the sample. For a total iron concentration of $5 \cdot 10^{13} \mathrm{~cm}^{-3}$, the equilibrium temperature is $T_{e q}^{\text {tot }} \approx 908^{\circ} \mathrm{C}$ and therefore $900^{\circ} \mathrm{C}<T_{e q}^{\text {tot }}<950^{\circ} \mathrm{C}, 1025^{\circ} \mathrm{C}$. Because for all annealing temperatures of the experiments, the solubility lies above the interstitial iron concentration of the as-grown wafer, i.e. $T>T_{e q}^{i}$, a dissolution of precipitates is observed during annealing as described in section 2.3 . The subsequent cooling rate and therefore the diffusional range was varied as shown in table 5. The influence of the cooling rate on the profiles will be discussed in this section.

The modeled concentrations of the as-grown simulations for a time of $17 \mathrm{~h}$ correspond to the measured as-grown states shown in figure 27 and are used as input for the simulations of the subsequent temperature treatments shown in figure 24. Since the experiments of fast and slow cooling at $900^{\circ} \mathrm{C}$ were done on neighbor wafers of the sample called 'LBIC 2' (see figure 25(b), the as-grown state 'LBIC 2' as shown in figure 27(b) was used for the corresponding simulations. For the simulations of slow cooling at $950^{\circ} \mathrm{C}$ and $1025^{\circ} \mathrm{C}$, the as-grown state named 'LBIC 1' shown in figure 27(a) was used, as the neighbor wafers of sample 'LBIC 1' (see figure 25(a)) were used for the experiments. Figure 30 shows the comparison of the line scans across the grain boundary for these temperature treatments for the modeled apparent interstitial iron concentration without and with the modeled recombination activity of the grain boundary (labeled 'Simulation' and 'LBIC simulation') and the calculated apparent interstitial iron concentration (labeled 'Experiment') calculated from the LBIC measurements by Krenckel [42].

The recombination velocities to fit the measured recombination effects of the grain boundaries are given in table 6.

\begin{tabular}{ll}
\hline \hline Simulation & $S_{e f f}\left[\frac{m}{s}\right]$ \\
\hline As grown LBIC 1 & 50 \\
As grown LBIC 2 & 200 \\
Fast $900^{\circ} C$ (LBIC 2, different line scan) & 500 \\
Slow $900^{\circ} C$ (LBIC 2) & 500 \\
Slow $950^{\circ} C$ (LBIC 1) & 200 \\
Slow $1025^{\circ} C$ (LBIC 1) & 200 \\
Slow $950^{\circ} C$ high N (LBIC 1) & 80 \\
Slow $1025^{\circ} C$ high N (LBIC 1) & 100
\end{tabular}

Table 6: Effective recombination velocities $S_{\text {eff }}$ for electrons at the grain boundary used in figures 28,30 and 32 .

The simulations fit well for the slow cooling after annealing at $900^{\circ} \mathrm{C}$ for $50 \mathrm{~min}$, while for the fast cooling and the annealing at high temperatures there is a discrepancy between simulations and experiment. 


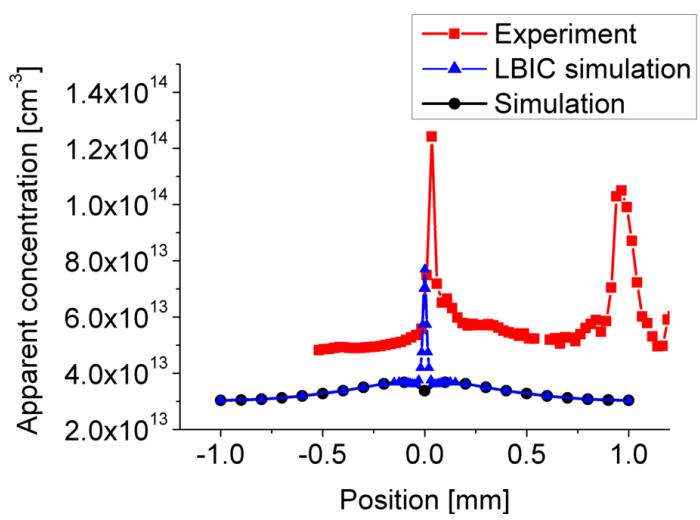

(a) Fast cooling after annealing at $900^{\circ} \mathrm{C}$ for $50 \mathrm{~min}$.

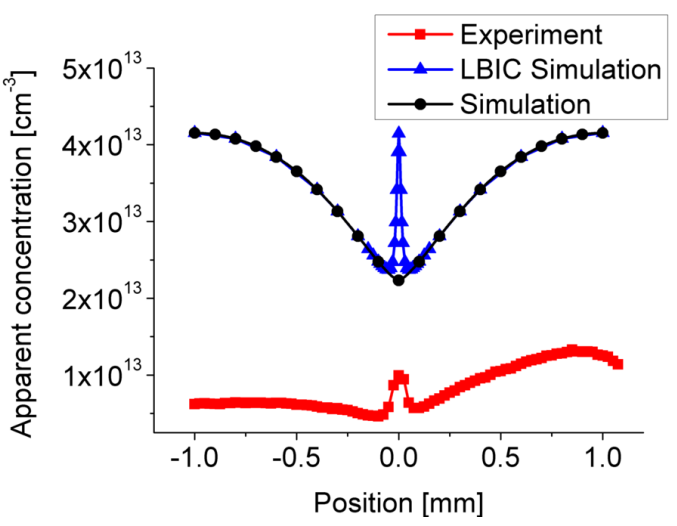

(c) Slow cooling after annealing at $950^{\circ} \mathrm{C}$ for 50 min.

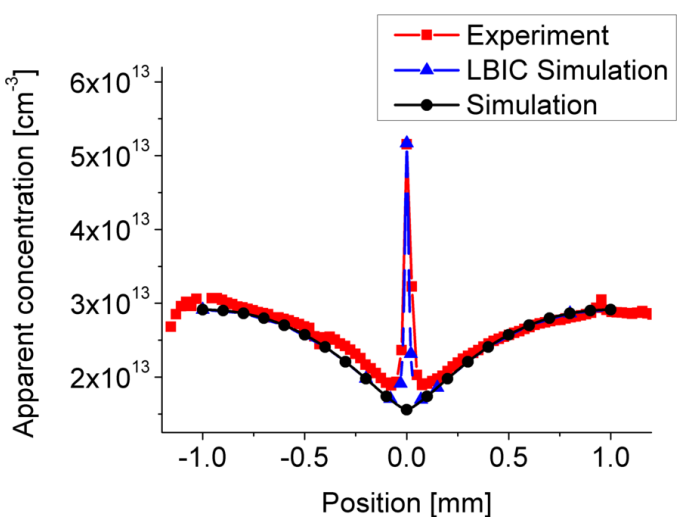

(b) Slow cooling after annealing at $900^{\circ} \mathrm{C}$ for $50 \mathrm{~min}$.

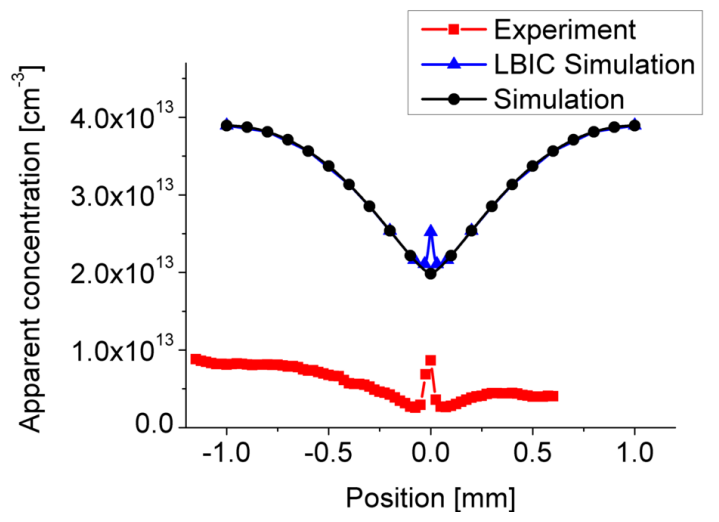

(d) Slow cooling after annealing at $1025^{\circ} \mathrm{C}$ for $50 \mathrm{~min}$.

Figure 30: Comparison of modeled and measured apparent interstitial iron concentrations. Shown is a line scan across a grain boundary for simulations and measurements of samples with different temperature treatments as shown in figure 24. The measured apparent concentrations are calculated from LBIC measurements by Krenckel [42]. There is a good agreement between modeled and measured line scans for low temperatures. For high temperatures there is a discrepancy between modeled and measured line scans. 
For fast cooling after $900^{\circ} \mathrm{C}$ for $50 \mathrm{~min}$ the diffusion length for the line scan across the grain boundary shown in figure 25(b) was not available and therefore a line scan across a different grain boundary had to be used. The position where the line scan was extracted instead is indicated in figure 25(b) by the light blue arrow (labeled 'Line scan 2'). The discrepancy of the modeled and the measured apparent concentrations can be explained by a difference in precipitation densities in the grains next to the two different grain boundaries and/ or different grain sizes.

The reason for the discrepancy between modeled and measured apparent concentrations for slow cooling after annealing at high temperatures of $950^{\circ} \mathrm{C}$ and $1025^{\circ} \mathrm{C}$ becomes understandable when comparing the evolution of the modeled interstitial iron concentrations with time for slow cooling after annealing at $900^{\circ} \mathrm{C}$ and $950^{\circ} \mathrm{C}$ as shown in figure 31

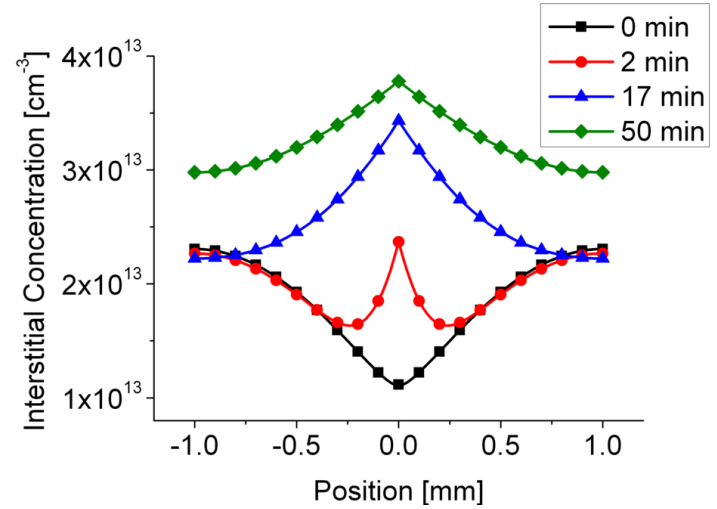

(a) Annealing at $900^{\circ} \mathrm{C}, C_{e q}=4.3 \cdot 10^{13} \mathrm{~cm}^{-3}$

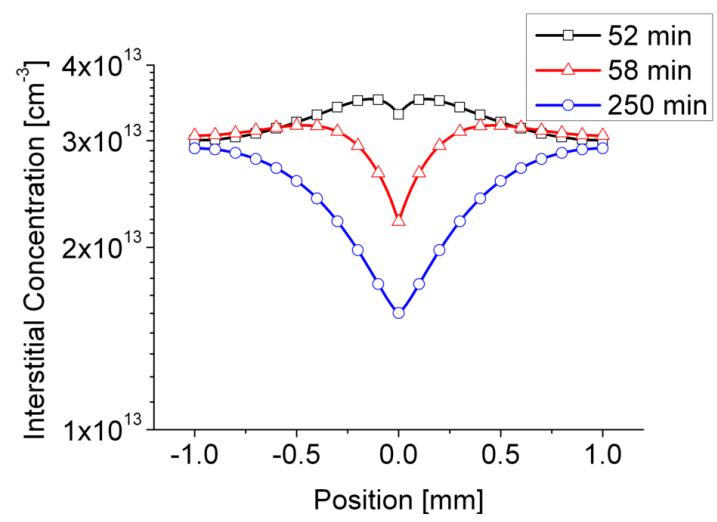

(c) Slow cooling after annealing at $900^{\circ} \mathrm{C}$

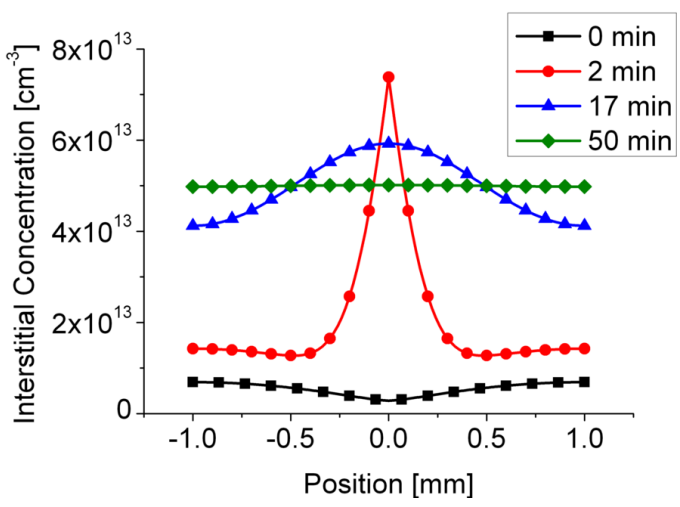

(b) Annealing at $950^{\circ} \mathrm{C}, C_{e q}=1.4 \cdot 10^{14} \mathrm{~cm}^{-3}$

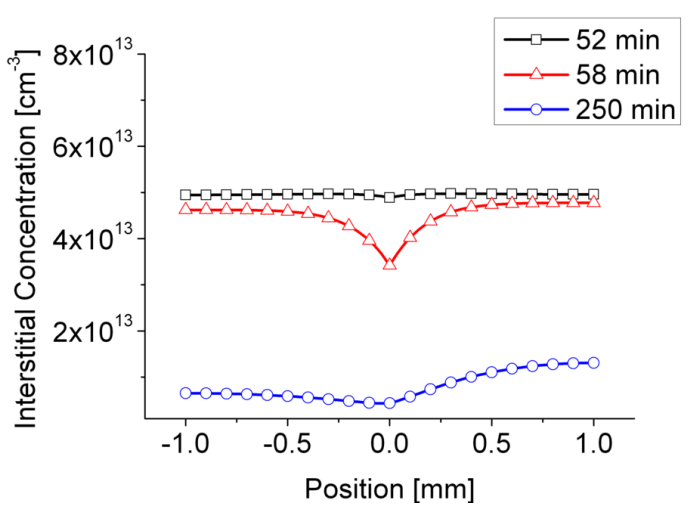

(d) Slow cooling after annealing at $950^{\circ} \mathrm{C}$

Figure 31: Time evolution of the interstitial iron concentration for annealing at $900^{\circ} \mathrm{C}$ and $950^{\circ} \mathrm{C}$ for $50 \mathrm{~min}$ and subsequent slow cooling.

Figures 31(a) and 31(b) show the annealing at $900^{\circ} \mathrm{C}$ and at $950^{\circ} \mathrm{C}$ for different times after the onset of annealing. The initial profile of the interstitial iron concentration at $t=0 \mathrm{~min}$ is the as-grown profile LBIC 2 in figure 27(b) for $900^{\circ} \mathrm{C}$ and the as-grown profile LBIC 1 in figure 
27 (a) for $950^{\circ} \mathrm{C}$. For reasons of comparison with the measured apparent interstitial iron concentrations, two different as-grown states were used. Both figures show the increasing interstitial iron concentration due to dissolution of precipitates at the grain boundary and in the grain. At $900^{\circ} \mathrm{C}$ the solubility of iron $C_{e q}$ lies below the total iron concentration present in the sample and it is not possible to dissolve all precipitates. As consequence, the profile in figure 31(a) shows that after 50min of annealing the interstitial iron concentration in the grain still lies below the total iron concentration of $5 \cdot 10^{13} \mathrm{~cm}^{-3}$. The dissolution of precipitates happens slowly and 50min of annealing are not sufficient to reach an equilibrium state, i.e. there is still a gradient of interstitial iron concentration present in the sample due to out-diffusion of impurities from the grain boundary. At $950^{\circ} \mathrm{C}$ the solubility of iron $C_{e q}$ lies above the total iron concentration present in the sample. The precipitates dissolve faster than at $900^{\circ} \mathrm{C}$ and $50 \mathrm{~min}$ of annealing are sufficient to distribute the interstitial iron concentration evenly in the sample at a level which equals the total iron concentration present in the sample, as can be seen in figure 31(b). Therefore, the initial as-grown state has no influence any more on the resulting line scan in contrast to the temperature treatments at $900^{\circ} \mathrm{C}$.

Figures 31(c) and 31(d) show slow cooling after 50min of annealing at $900^{\circ} \mathrm{C}$ and $950^{\circ} \mathrm{C}$, respectively. Both figures show a decreasing interstitial iron concentration due to the precipitation in the grains and the formation of the denuded zones due to precipitation at the grain boundary until the measured concentration profiles are reached. For $900^{\circ} \mathrm{C}$ not all precipitates are dissolved after 50min of annealing. During cooling the interstitial iron precipitates at the still existing precipitates. For $950^{\circ} \mathrm{C}$ all precipitates are dissolved and during cooling new precipitates form. The slow cooling has a higher cooling rate than the cooling after crystallization (compare table 6), which causes a higher level of supersaturation and therefore a higher precipitation density. A higher precipitation density for a higher level of supersaturation has been observed experimentally [77, 53].

Therefore, for the simulation of cooling after $950^{\circ} \mathrm{C}$ annealing, a higher precipitation density of $N_{p}=3.7 \cdot 10^{8} \mathrm{~cm}^{-3}$ in the left grain, $N_{p}=2 \cdot 10^{8} \mathrm{~cm}^{-3}$ in the right grain and $N_{g b}=3.5$. $10^{7} \mathrm{~cm}^{-2}$ at the grain boundary are used. The qualitative difference for $900^{\circ} \mathrm{C}$ and $950^{\circ} \mathrm{C}$ is due to the equilibrium temperature $T_{e q}^{t o t}$ at which the solubility equals the total iron concentration of $5 \cdot 10^{13} \mathrm{~cm}^{-3}$ in the sample lying at $T_{e q}^{\text {tot }} \approx 908^{\circ} \mathrm{C}$ and separating the two temperatures $900^{\circ} \mathrm{C}<$ $T_{e q}^{t o t}<950^{\circ} \mathrm{C}$. 
Figure 32 shows that the modeled concentration profiles for high temperatures of $950^{\circ} \mathrm{C}$ and $1025^{\circ} \mathrm{C}$ fit well to the measured concentration profiles when changing the precipitation densities to higher values.

For the simulation of $1025^{\circ} \mathrm{C}$ a precipitation density of $N_{p}=2.3 \cdot 10^{8} \mathrm{~cm}^{-3}$ in the left grain, $N_{p}=3 \cdot 10^{8} \mathrm{~cm}^{-3}$ in the right grain and $N_{g b}=1 \cdot 10^{8} \mathrm{~cm}^{-2}$ at the grain boundary are used. The used recombination velocities at the grain boundary are give in table 6 .

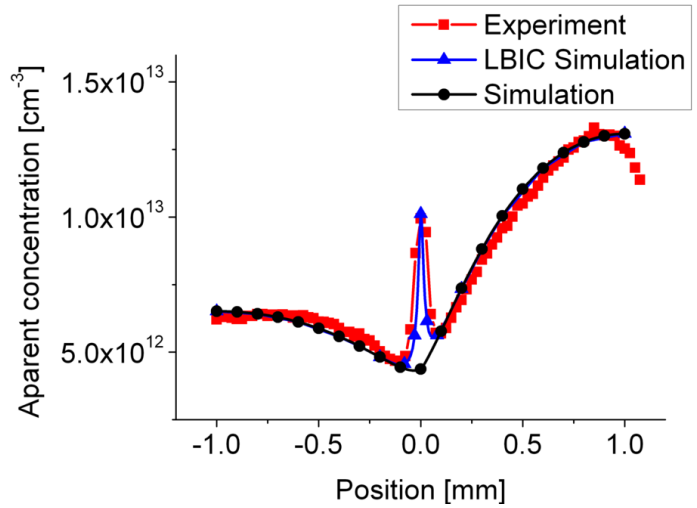

(a) Slow cooling after annealing at $950^{\circ} \mathrm{C}$ for $50 \mathrm{~min}$

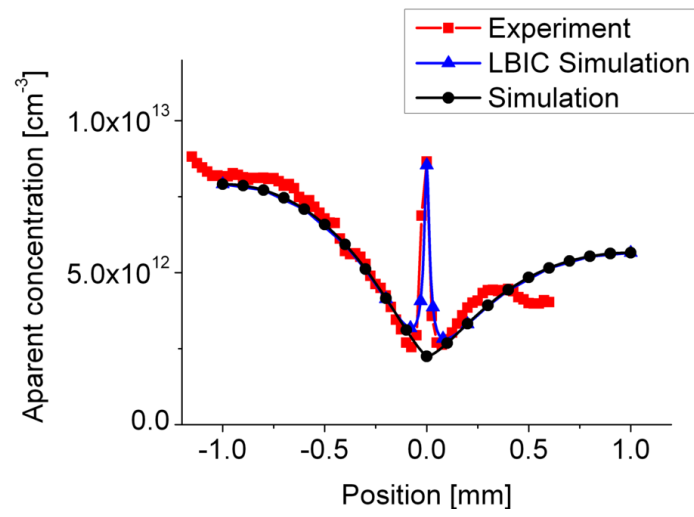

(b) Slow cooling after annealing at $1025^{\circ} \mathrm{C}$ for $50 \mathrm{~min}$

Figure 32: Modeled and measured line scans for the apparent interstitial iron concentrations for simulations with higher precipitate densities.

Figure 33 illustrates the influence of the as-grown state on the final interstitial iron concentration profile for $900^{\circ} \mathrm{C}$ after slow cooling. It shows the profiles of the interstitial iron concentration for the simulation with the as-grown concentrations as input and a simulation with a constant interstitial iron concentration of $4.26 \cdot 10^{13} \mathrm{~cm}^{-3}$ according to the solubility of iron at $900^{\circ} \mathrm{C}$. There is a difference in the level of the interstitial iron concentration in the grains and in the shape of the denuded zone. Simulations have shown that this difference becomes more significant for larger grains.

Figure 33 points out that for temperature treatments with a temperature $T<T_{e q}^{t o t}$ it is important to take the history of temperature treatments of the sample into account. Another example of the influence of the initial state on the final profile is the fast cooled sample. A comparison of the profiles of the interstitial iron concentration of the fast cooled sample before and after annealing at $900^{\circ} \mathrm{C}$ in figure 34 shows that almost no precipitation takes place and the fast cooling has only little effect on the final interstitial iron concentration profile in the vicinity of the grain boundary.

The diffusional range of table 5 fits with $R_{M}=0.16 \mathrm{~mm}$ well to the width of the denuded zone of the profile after cooling in figure 34. The width of the denuded zone is in the same range as the peak originating from the recombination activity of the grain boundary in the measured and modeled line scan in figure 30(a) and therefore can not be detected in the measurements. The line scan measured after the fast cooling therefore show the 'frozen' state of the sample after 


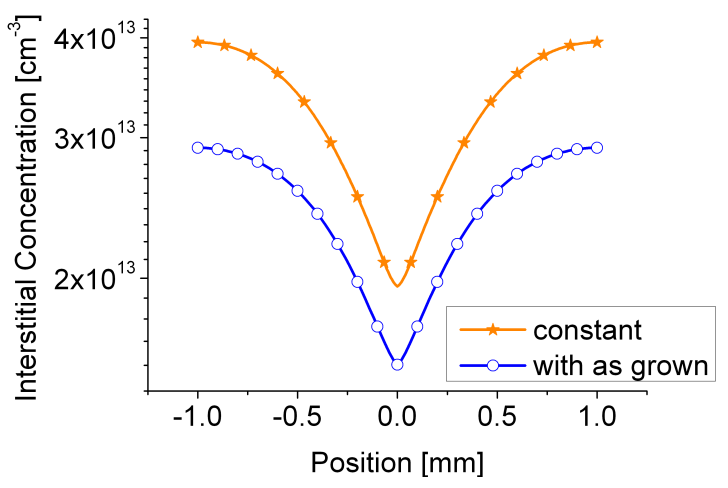

Figure 33: Profiles of the interstitial iron concentration for a simulation with the as-grown state and with a constant value of $5 \mathrm{e} 13 \mathrm{~cm}^{-} 3$ as initial state.

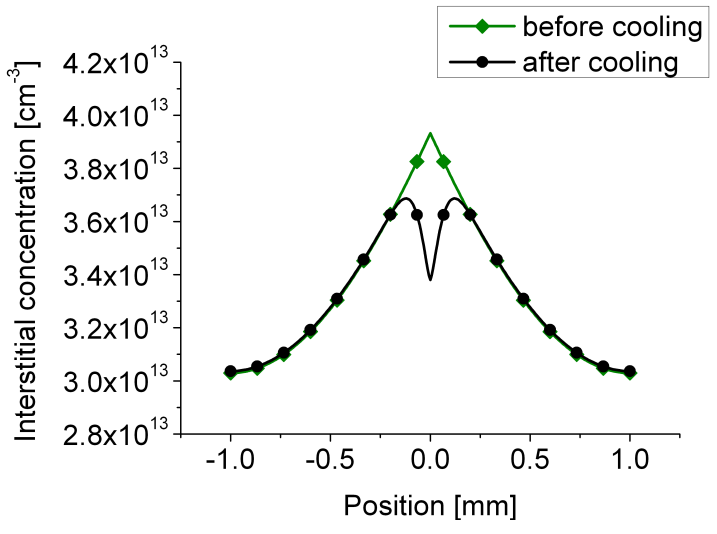

Figure 34: Profiles of the interstitial iron concentration for fast cooling after 50min annealing at $900^{\circ} \mathrm{C}$ before and after cooling. 
annealing.

The comparison of the denuded zones of all measured and modeled line scans in this section with the calculated diffusional ranges in table 5 indicate that the accumulation at grain boundaries via precipitation is mainly diffusion-limited for the investigated cooling processes. Diffusion-limited precipitation at grain boundaries has also been observed in experiments for low temperatures [102, 15]. 


\subsubsection{Simulations for comparison with PL measurements}

In Liu et al. [35] and Liu and Macdonald [53] the authors present PL measurements on grain boundaries of neighbor wafers with different temperature treatments. The wafers with a thickness of $330 \mu \mathrm{m}$ are from a near bottom region of a boron-doped multicrystalline silicon ingot which was grown commercially by directional solidification. The total iron concentration was interpolated by Liu [103] to $1 \cdot 10^{14} \mathrm{~cm}^{-3}$ from the iron versus ingot height plot derived from NAA measurements by Macdonald et al. [6]. In Liu et al. [35] the as-cut neighbor wafers were annealed at different temperatures for different annealing times and in Liu and Macdonald [53] annealed at a temperature of $500^{\circ} \mathrm{C}$ for different annealing times. Figure 35 shows the temperature curves according to the descriptions in Liu and Macdonald [53] and Liu et al. [35] which were used as input for simulations. Figure 35(c) shows the assumed temperature curve for the cooling after crystallization according to Liu [103].

For the annealing temperatures of $T=500^{\circ} \mathrm{C}$ and $600^{\circ} \mathrm{C}$, the solubility of interstitial iron in silicon is smaller than the total iron concentration and even smaller than the interstitial iron concentration measured by PL imaging. Therefore, during the annealing at $500^{\circ} \mathrm{C}$ and $600^{\circ} \mathrm{C}$, there is no dissolution of precipitates, but further precipitation occurs. For the annealing at $T=800^{\circ} \mathrm{C}$ the solubility lies within the range of the interstitial iron concentration of the initial state of the sample. After the comparison of simulations and experimental data for this annealing temperature, the behavior at this temperature will be discussed. For the annealing temperatures of $T=900^{\circ} \mathrm{C}$ and $1000^{\circ} \mathrm{C}$, the solubility of interstitial iron in silicon is smaller than the interstitial iron concentration of the initial state of the sample, i.e. $T>T_{e q}^{i}$. In this temperature regime dissolution of precipitates is expected during annealing. All samples (except the as-cut sample) were cooled on air with a cooling rate of approximately $0.3 \frac{K}{s}$, which is comparable to the fast cooling described in section 5.3.1 above. It was shown in section 5.3.2 that this fast cooling has almost no influence on the concentration profiles because only very little precipitation takes place during the short time period of cooling. This is also supported by the calculated diffusional ranges $R_{M}$ of iron, i.e. the mean distance the interstitial iron travels during the cooling processes. The diffusional ranges during cooling $R_{M}$ and during annealing $R_{M}^{a}$ calculated by a numerical evaluation based on equation 3 are given in table 7 . The table shows that the diffusional ranges during the cooling process are small for all cooling processes especially compared to the diffusional range during annealing. The only exception is the diffusional range of the cooling process of the as-cut sample. Therefore, the concentration profiles in this section are expected to show the 'frozen' state after annealing, except in the very close vicinity of the grain boundary. 


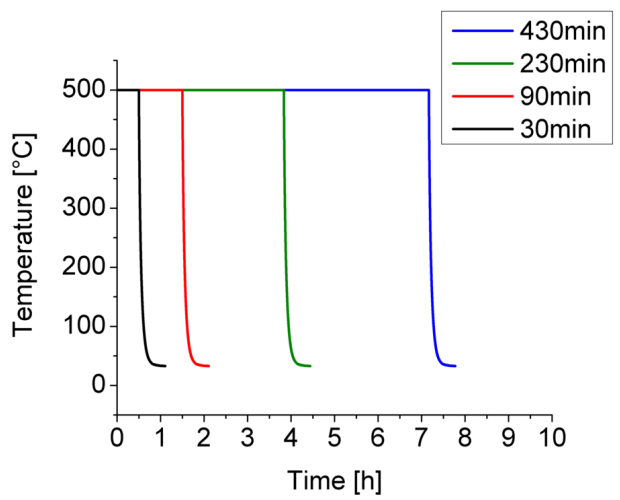

(a) Temperature curve for the wafer with grain boundary 'GB1'

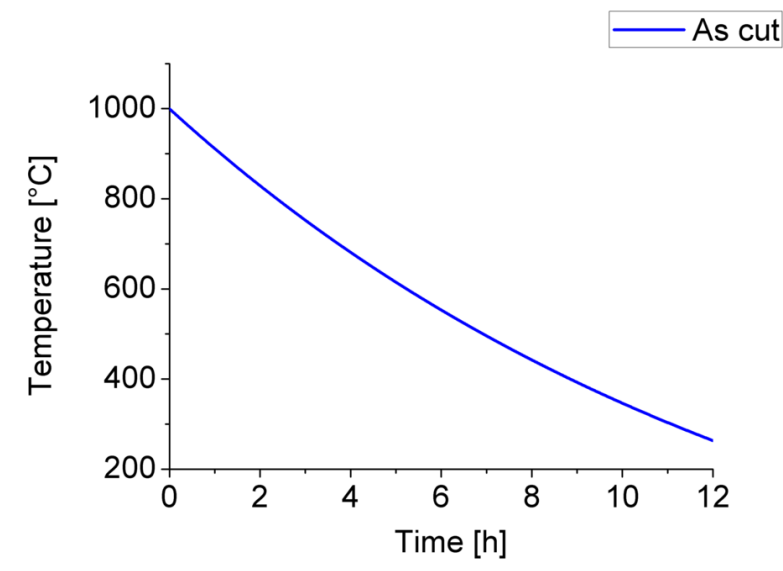

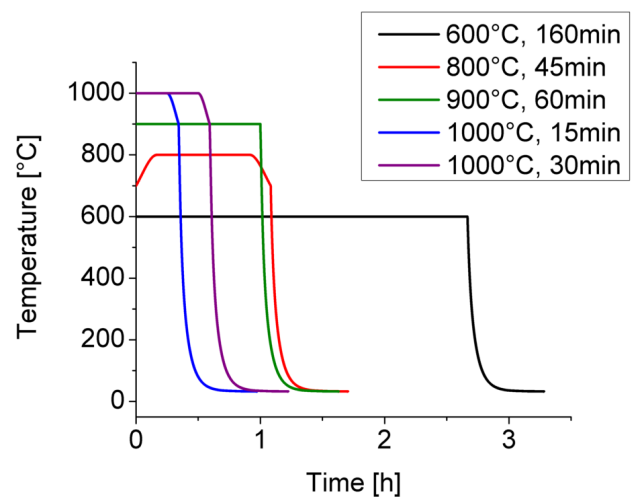

(b) Temperature curves for the wafer with grain boundary 'GB2'

(c) As cut temperature curve for the wafer with grain boundary 'GB2'

Figure 35: Temperature treatments used to model the experiments performed on two different sets of neighbor wafers by Liu and Macdonald [53] and Liu et al. [35]. Figure 35(a) shows a variation of annealing time at a constant annealing temperature. Figure 35(b)] shows a variation of annealing temperature and time. Figure 35(c) shows the assumed temperature curve for the as-cut sample. 


\begin{tabular}{llll}
\hline \hline Simulation & Cooling rate $\beta\left[\frac{K}{s}\right]$ & Diffusional range $R_{M}[\mathrm{~mm}]$ & Diffusional range $R_{M}^{a}$ \\
\hline As cut & 0.017 & 2.8 & - \\
$500,30 \mathrm{~min}$ & 0.3 & 0.02 & 0.23 \\
$500,90 \mathrm{~min}$ & 0.3 & 0.02 & 0.39 \\
$500,230 \mathrm{~min}$ & 0.3 & 0.02 & 0.63 \\
$500,430 \mathrm{~min}$ & 0.3 & 0.02 & 0.86 \\
$600,160 \mathrm{~min}$ & 0.3 & 0.05 & 0.94 \\
$800,45 \mathrm{~min}$ & 0.3 & 0.07 & 0.80 \\
$900,60 \mathrm{~min}$ & 0.3 & 0.16 & 1.84 \\
$1000,15 \mathrm{~min}$ & 0.3 & 0.16 & 1.19 \\
$1000,30 \mathrm{~min}$ & 0.3 & 0.16 & 1.69
\end{tabular}

Table 7: Diffusional ranges during cooling $R_{M}$ and during annealing $R_{M}^{a}$ for the simulations with the temperature curves from figure 35 . 
The two dimensional maps in figure 36 taken from Liu and Macdonald [53] and Liu et al. [35] show the measured 'apparent interstitial iron concentrations' calculated from PL measurements as described in section 2.6. Dark features indicate a low concentration and bright contrast a high concentration of interstitial iron concentration. We chose the term 'apparent interstitial iron concentrations' because several assumptions have to be made for the calculation of the concentration from the PL signal similar to LBIC as described in section 2.6 (see also Macdonald et al. [40] for more detail). Also it is not exactly understood yet, if the calculation is correct in the region very close to the grain boundary [80].

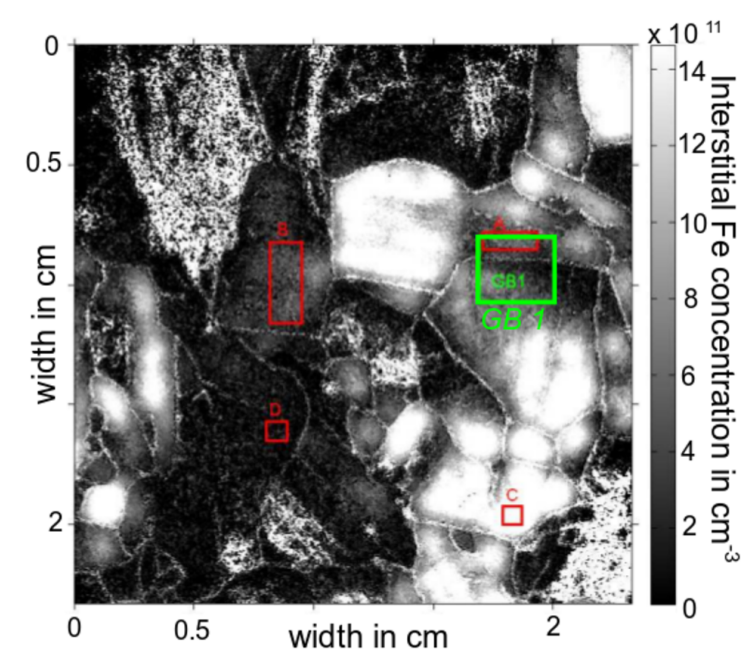

(a) Wafer with grain boundary 'GB1'

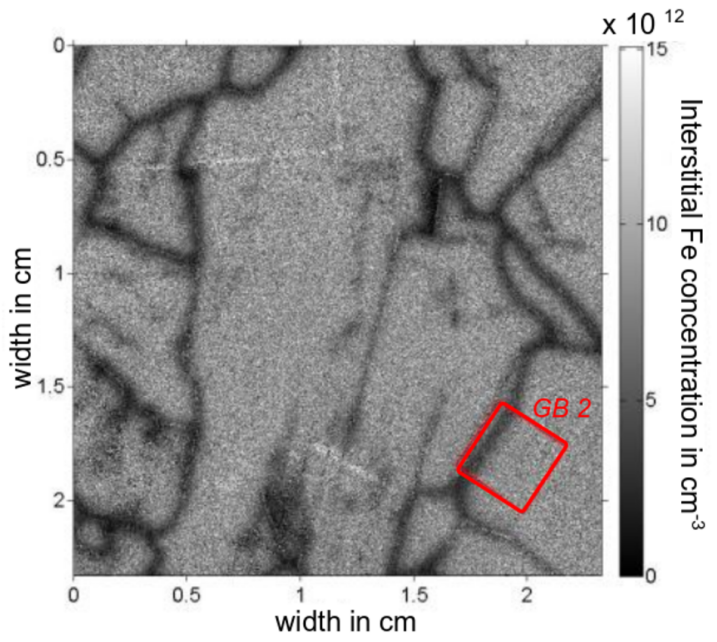

(b) Wafer with grain boundary 'GB2'

Figure 36: Apparent interstitial iron concentration calculated from PL measurements. Figure 36(a) is taken from Liu and Macdonald [53] and shows a wafer after annealing at $500^{\circ} \mathrm{C}$ for 867 minutes. Figure 36(b) is taken from Liu et al. [35] and shows a wafer in the as-cut state.

Figure 36(a) shows a wafer after annealing at $500^{\circ} \mathrm{C}$ for $867 \mathrm{~min}$ and subsequent fast cooling. It shows grains with different concentrations and denuded zones in the vicinity of the grain boundaries. The green rectangle in figure 36(a) marks the region, where a line scan across the grain boundary was extracted. This grain boundary will be denoted as 'GB1' in the following text. Figure 36(b) shows a wafer in the as-cut state. The red rectangle marks the region, where the line scans across the grain boundary, denoted as 'GB2' in the further text, were extracted.

The model geometry chosen for GB1 and GB2 can be seen in figure 37. It shows two dimensional maps of the modeled interstitial iron concentration from a model with only precipitation as mechanism of impurity accumulation at the grain boundary. Figure 37(a) shows the results for a simulation with annealing at $500^{\circ} \mathrm{C}$ for $30 \mathrm{~min}$ for GB1. Figure $\left.37(\mathrm{~b})\right]$ shows the modeled as-cut state of GB2 with the temperature curve for cooling after crystallization as input. The grain size was chosen to match the geometry of the grain boundaries marked with the green (for GB1) and the red (for GB2) rectangles in figure 36. In contrast to the previous sample geometry in section 5.3.1, the grain boundary separates two grains of different size namely $0.8 \mathrm{~mm}$ and $0.2 \mathrm{~mm}$ for 
GB1 and $0.2 \mathrm{~mm}$ and $1 \mathrm{~mm}$ for GB2. The wafer thickness was chosen to $330 \mu \mathrm{m}$ and the grain boundary is positioned at $x=0 \mathrm{~mm}$ as indicated by the black arrows. The total iron concentration was set to $1 \cdot 10^{14} \mathrm{~cm}^{-3}$, as this value is interpolated by Liu [103] from the iron versus ingot height plot derived from NAA measurements by Macdonald et al. [6]. For the simulation of the as-cut sample (figure 37(b)) it was assumed that at $1300^{\circ} \mathrm{C}$ the total iron concentration of $1 \cdot 10^{14}$ is dissolved. The precipitate densities in the grain are set to $N_{p}=1.2 \cdot 10^{8} \mathrm{~cm}^{-3}$ for GB1 and $N_{p}=9.5 \cdot 10^{6} \mathrm{~cm}^{-3}$ for GB2. The precipitate densities at the grain boundary are chosen to $N_{g b}=5 \cdot 10^{5} \mathrm{~cm}^{-2}$ for GB1 and $N_{g b}=1.6 \cdot 10^{6} \mathrm{~cm}^{-2}$ for GB2.

The initial state for the annealing at $500^{\circ} \mathrm{C}$, i.e. for the temperature treatments performed on GB1 is an oxidized sample with a homogeneous initial concentration of $1 \cdot 10^{13} \mathrm{~cm}^{-3}$ in the grains, except in the very close vicinity of grain boundaries (please see figure 38(a)). For all temperature processes performed on GB2 the initial state is the as-cut sample shown in figure 37(b). The interstitial iron concentration for the as-cut wafer ranges from $3.5-8.5 \cdot 10^{12} \mathrm{~cm}^{-3}$.

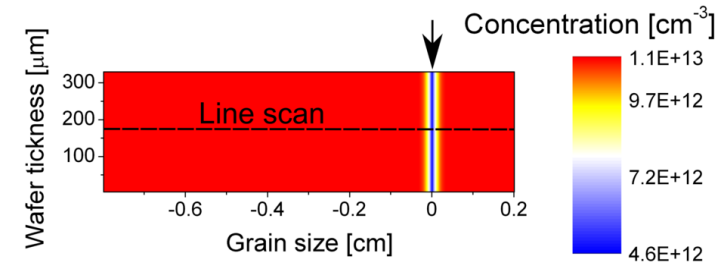

(a) $2 \mathrm{~d}$ map of GB1 after $30 \mathrm{~min}$ at $500^{\circ} \mathrm{C}$

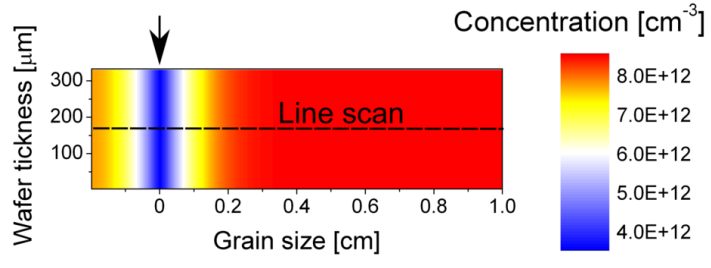

(b) $2 \mathrm{~d}$ map of GB2 in the as-cut state

Figure 37: Two dimensional maps of the interstitial iron concentration of the simulations for GB1 and GB2. 


\subsubsection{Simulations of precipitation and dissolution of precipitates for comparison with PL measurements}

During annealing at a temperature with $T<T_{e q}^{i}$, i.e. when the interstitial iron concentration of the initial state lies above the solubility, precipitation at the grain boundary and in the grains occurs. This is the case in the experiments for an annealing temperature of $500^{\circ} \mathrm{C}$ (GB1) and $600^{\circ} \mathrm{C}$ (GB2) described in Liu and Macdonald [53] and Liu et al. [35], as already stated in 5.3.3. An approximation of the expected width of the denuded zone in the line scans can be derived from the sum of the diffusional ranges during annealing and cooling in table 7. Please not that the diffusional range during cooling is small compared to the diffusional range during annealing.

Figure 38 shows the comparison of the modeled interstitial iron concentration and the apparent interstitial iron concentration for the temperature treatments shown in figure 35(a), i.e. a variation of annealing time at a temperature of $500^{\circ} \mathrm{C}$ and a subsequent fast cooling. The concentrations of the oxidized sample were used as input for the simulations. The figures show that the modeled profiles agree well with the measured PL profiles, except in the close vicinity of the grain boundary. In some line scans from the PL measurements, there is an enhanced iron concentration in the close vicinity of the grain boundary (see e.g. figure $38(\mathrm{~d})$ ) similar to the profiles derived from LBIC measurements. It is not readily understood, if this is a real concentration or a measurement artifact (see [35, 80]. Also the question remains why this enhanced concentration appears only in some of the line scans. In the grain not too close to the grain boundary the measured line scans can be reproduced by the modeled line scans and also the denuded zones agree well for an annealing temperature of $500^{\circ} \mathrm{C}$ for different annealing times and subsequent fast cooling. As the figures 38(a) to 38(d) show increasing annealing times with fast cooling and the precipitation during cooling is negligible compared to the long precipitation time during annealing, the figures show the time evolution of the apparent interstitial iron concentration during annealing: with increasing annealing time the interstitial iron concentration decreases due to precipitation within the grains and the width of the denuded zone increases due to precipitation at the grain boundary. 


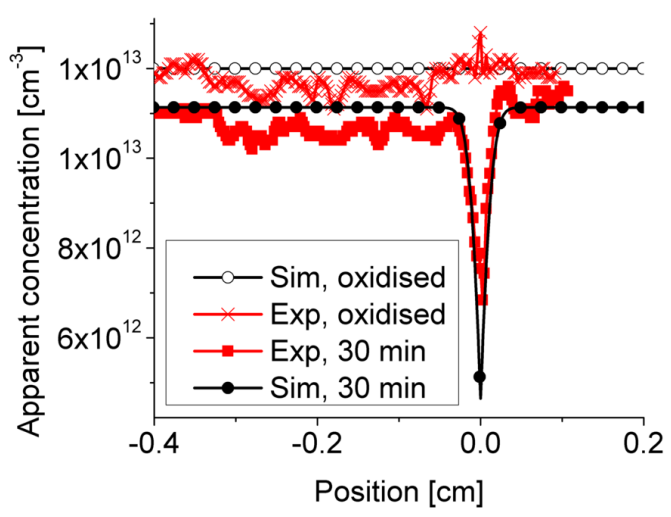

(a) Annealing at $500^{\circ} \mathrm{C}$ for $30 \mathrm{~min}$

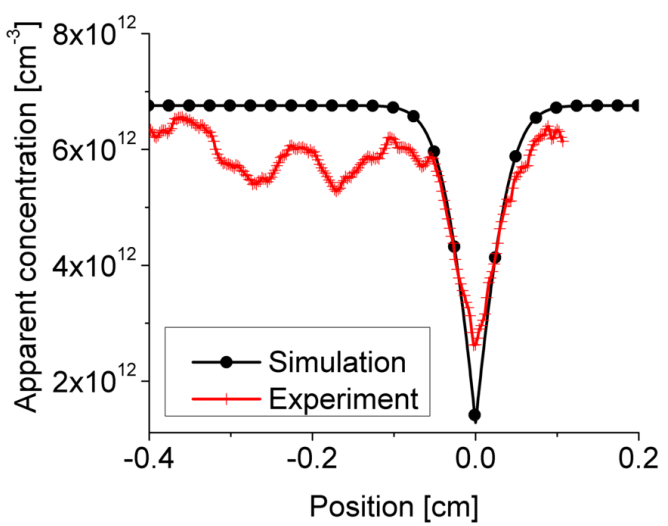

(c) Annealing at $500^{\circ} \mathrm{C}$ for $230 \mathrm{~min}$

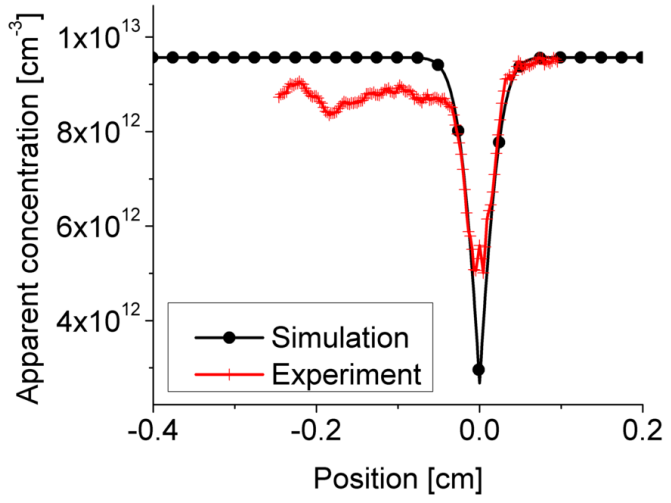

(b) Annealing at $500^{\circ} \mathrm{C}$ for $90 \mathrm{~min}$

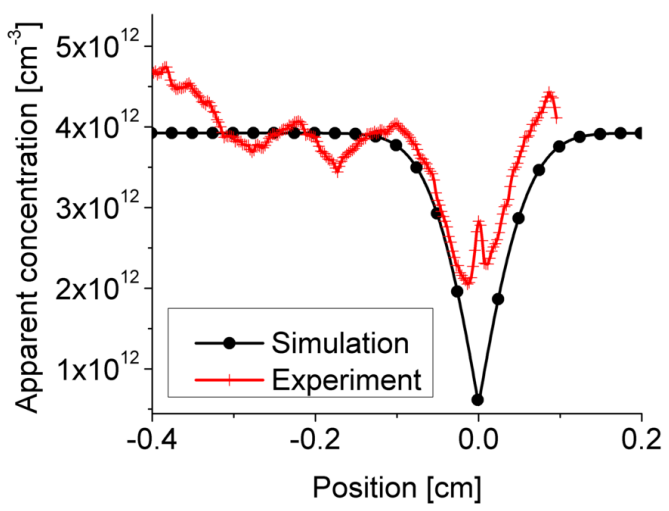

(d) Annealing at $500^{\circ} \mathrm{C}$ for $430 \mathrm{~min}$

Figure 38: Comparison of the line scans across the grain boundary for the modeled and measured apparent interstitial iron concentration after different durations of annealing at $500^{\circ} \mathrm{C}$ performed on the wafers with grain boundary GB1. Modeled and measured line scans are in close accordance. 
Figure 39 shows the comparison of the modeled and measured apparent interstitial iron concentrations for the grain boundary marked with the red rectangle in figure $36(\mathrm{~b})$ for a variation of annealing temperature and time with subsequent fast cooling according to the temperature curves shown in figure $35(\mathrm{~b})$. The position of the modeled line scans is indicated by the black line in figure 37(b),

Figure 39(a) shows the modeled and measured line scans across the grain boundary for the as-cut state. While it agrees well in the grain far away from the grain boundary, the denuded zone in the modeled line scan is wider than in the measured line scan. Also in the measured line scans, there is an enhanced concentration in the close vicinity of the grain boundary of uncertain origin as already mentioned in the comparison of the line scans for GB1 (see figure 38). For the simulations of the other temperature curves the concentrations of the as-cut state are used as input. The modeled and measured line scans of GB2 after annealing at $600^{\circ} \mathrm{C}$ for $160 \mathrm{~min}$ shown in figure 39(b) fit well. Similar to the annealing at $500^{\circ} \mathrm{C}$ the line scans illustrate how the interstitial iron concentration has -compared to the as-cut state- decreased in the grains and the depth of the denuded zone has increased due to precipitation in the grains and at the grain boundary. In the line scans for annealing at $800^{\circ} \mathrm{C}$ for $45 \mathrm{~min}$, a clear discrepancy is seen between the modeled and measured apparent interstitial iron concentrations. While the measured line scan looks very similar to the as-cut state, i.e. no precipitation seems to occur in the grains or at the grain boundary, the modeled apparent interstitial iron concentration decreases in comparison to the as-cut state similar to the simulation for annealing at $600^{\circ} \mathrm{C}$.

For annealing temperatures with $T>T_{e q}^{i}$, i.e. when the interstitial iron concentration of the initial state lies below the solubility, dissolution of precipitates is expected during annealing. This is the case in the experiments for an annealing temperature of $900^{\circ} \mathrm{C}$ and $1000^{\circ} \mathrm{C}$. During subsequent cooling, precipitation occurs. However for the fast cooling used here, the precipitation during cooling has only minor effect on the resulting concentration profiles and the denuded zone resulting from the cooling process lie in the range of the peak of uncertain origin in the close vicinity of the grain boundaries.

The simulations at high temperatures of $900^{\circ} \mathrm{C}$ and $1000^{\circ} \mathrm{C}$ in figure 39(d), 39(e) and 39(f) show up to one order of magnitude higher interstitial iron concentrations than the measured line scans. The discrepancy between measured and modeled line scans for temperature above $600^{\circ} \mathrm{C}$ indicate that the initial total iron concentration in the sample is much lower than the assumed total iron concentration of $1 \cdot 10^{14} \mathrm{~cm}^{-3}$. Also the line scan for annealing at $1000^{\circ} \mathrm{C}$ for $30 \mathrm{~min}$ indicates that the total iron concentration is lower because after an annealing time of $30 \mathrm{~min}$ at a temperature of $1000^{\circ} \mathrm{C}$, nearly all iron is expected to be dissolved and the profile should have a homogeneous value equaling the total iron concentration. The corresponding line scan in figure 39(f) shows such a homogeneous concentration profile, except very close to the grain boundary. The peak at the grain boundary could be an indication that not all iron is dissolved after the $30 \mathrm{~min}$ annealing step. However, by comparison to the other line scans (see e.g. the line scan in figure 38(d)], one can assume that the peak originates from the measurement artifact discussed above and does not show a real interstitial iron concentration. In this case the level with $1 \cdot 10^{13} \mathrm{~cm}^{-3}$ in the line scan in figure 39(f) gives the amount of total iron concentration in the sample. There- 


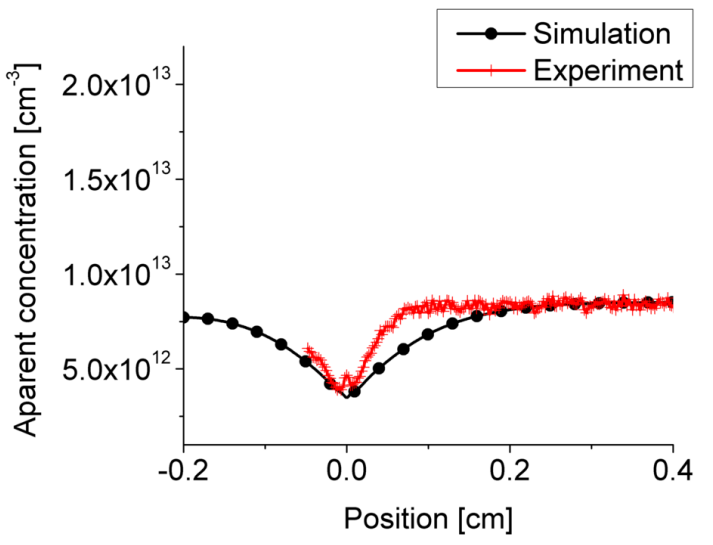

(a) As cut

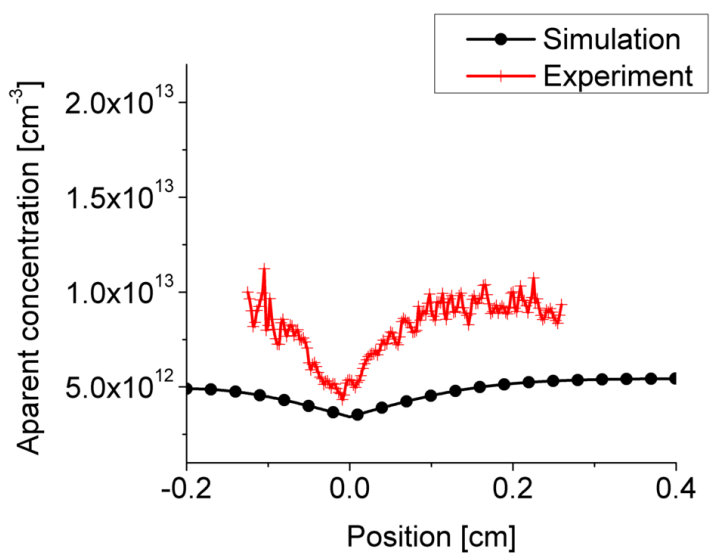

(c) Annealing at $800^{\circ} \mathrm{C}$ for $45 \mathrm{~min}$

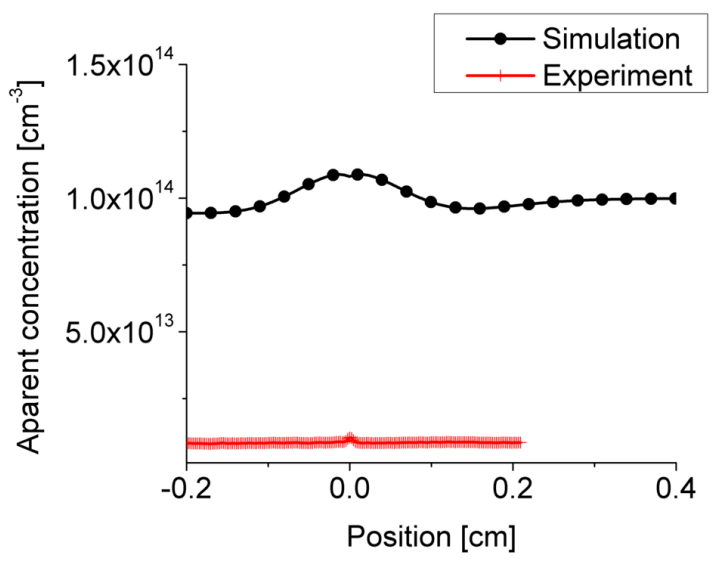

(e) Annealing at $1000^{\circ} \mathrm{C}$ for $15 \mathrm{~min}$

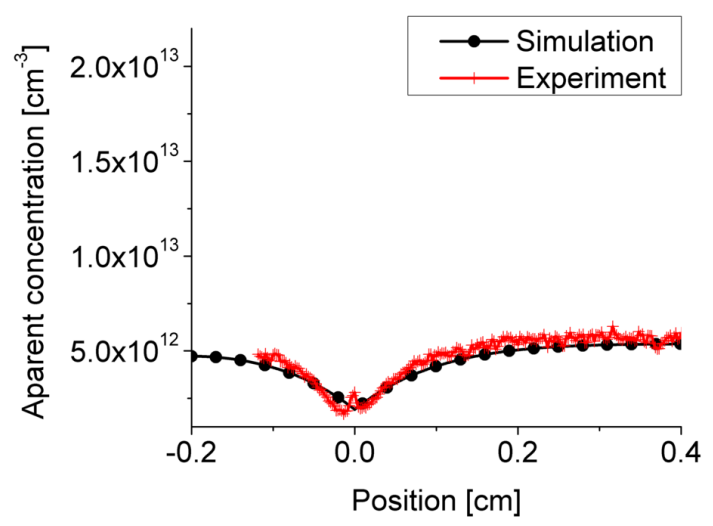

(b) Annealing at $600^{\circ} \mathrm{C}$ for $160 \mathrm{~min}$

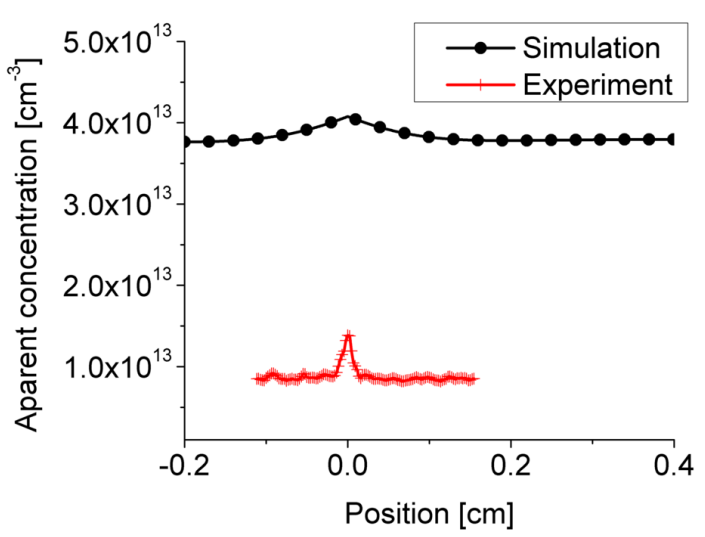

(d) Annealing at $900^{\circ} \mathrm{C}$ for $60 \mathrm{~min}$

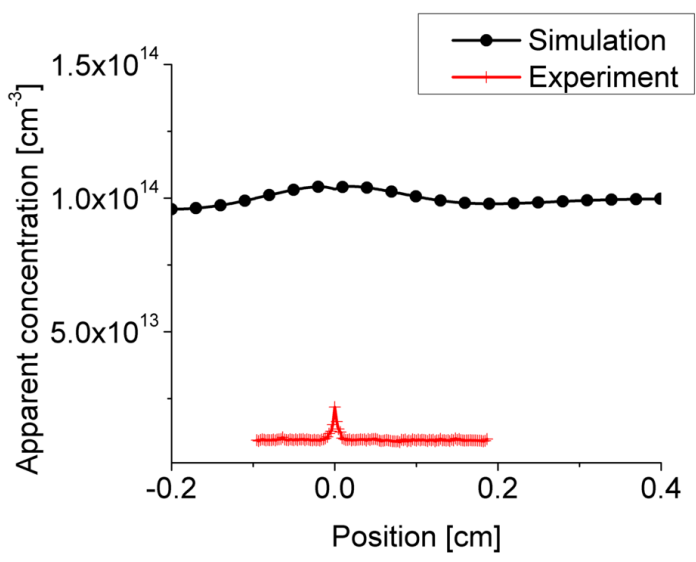

(f) Annealing at $1000^{\circ} \mathrm{C}$ for $30 \mathrm{~min}$

Figure 39: Comparison of the line scans across the grain boundary for the modeled and measured apparent interstitial iron concentration for different annealing temperatures and annealing times performed on the wafer with the grain boundary GB2. For high temperatures there is a discrepancy between modeled and measured line scans. 
fore, in the following, simulations with a lower total iron concentration of $1 \cdot 10^{13} \mathrm{~cm}^{-3}$ will be compared to the measured line scans.

Figure 40 shows the results for a simulation with a total iron concentration of $1 \cdot 10^{13} \mathrm{~cm}^{-3}$ and the temperature curve shown in figure 24, which was used for the simulation of the asgrown sample in section 5.3.1. The precipitate density is $N_{p}=5 \cdot 10^{8} \mathrm{~cm}^{-3}$ in the grains and $N_{g b}=1 \cdot 10^{8} \mathrm{~cm}^{-2}$ at the grain boundary and was chosen to fit the measured line scans for $600^{\circ} \mathrm{C}$.

As can be seen the measured and modeled line scans for high temperatures fit much better. The still existing discrepancy can be explained by the lack of knowledge of the cooling curve and therefore an incorrect fitting of the precipitate densities. The discrepancy of the modeled and measured line scans for the as-cut state shows that the combination of cooling curve and precipitate densities at the grain boundary and in the grain does not exactly match the experimental conditions. However, the diffusional range calculated with the as-grown temperature profile of figure 24, assuming a total iron concentration of $1 \cdot 10^{13} \mathrm{~cm}^{-3}$ is $R_{M}=0.9 \mathrm{~mm}$, which fits much better to the width of the denuded zone in the experimental line scan of the as-cut sample in figure 40(a) than the diffusional range calculated for a concentratio of $1 \cdot 10^{14} \mathrm{~cm}^{-3}$. For the high temperatures the slightly incorrect fitting of the precipitate densities results in slightly different concentration levels.

For the line scan for annealing at $800^{\circ} \mathrm{C}$ not only the incorrect precipitate densities, but also the different as-cut state - which serves as initial state of the simulation - can result in a discrepancy. For $800^{\circ} \mathrm{C}$ the solubility is with approximately $4.6 \cdot 10^{12} \mathrm{~cm}^{-3}$ in the range of the interstitial iron concentration of the as-cut sample, which amounts $8.5-3.5 \cdot 10^{12} \mathrm{~cm}^{-3}$. In this temperature regime close to the equilibrium temperature, the line scan depends very sensitively on the initial conditions, as is illustrated on the example of two simulations with slightly different temperatures of $800^{\circ} \mathrm{C}$ and $820^{\circ} \mathrm{C}$ in figure 40(c). Furthermore, for a temperature for which the solubility equals the interstitial iron concentration present in the sample, no precipitation or dissolution, but precipitate ripening (Ostwald ripening) is expected, which is not included in the model. This is probably the major reason for the discrepancy at $800^{\circ} \mathrm{C}$. 


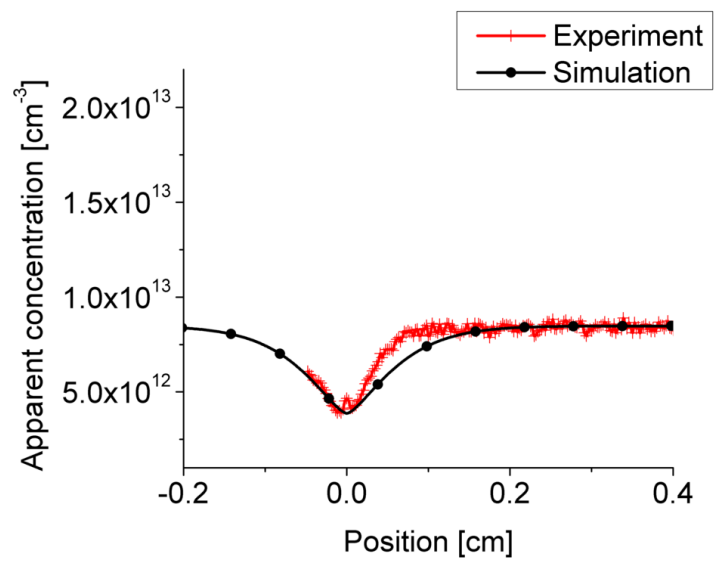

(a) As cut

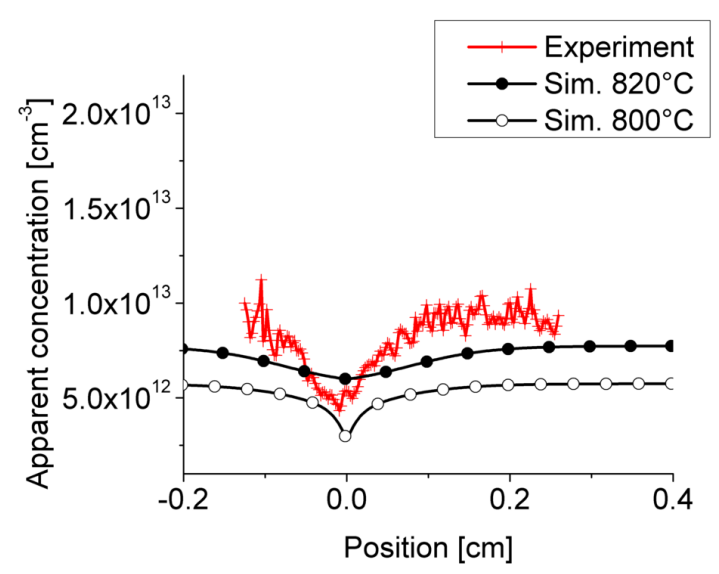

(c) $800^{\circ} \mathrm{C}$ and $820^{\circ} \mathrm{C}$ for $45 \mathrm{~min}$

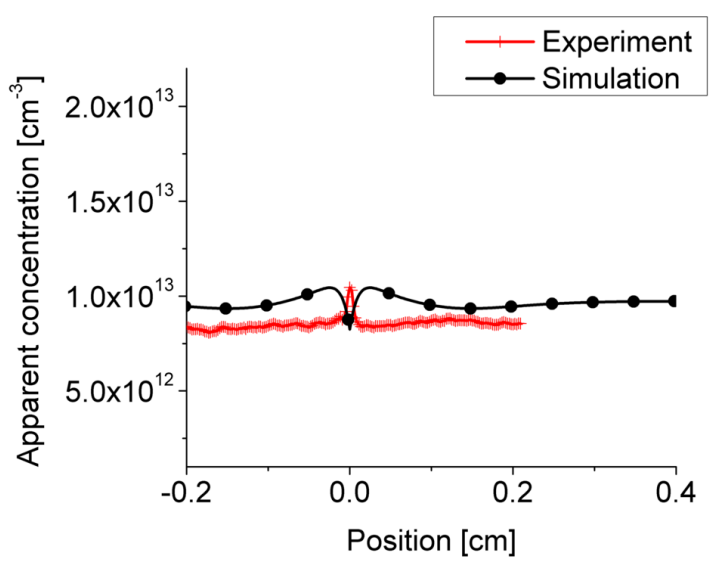

(e) $1000^{\circ} \mathrm{C}$ for $15 \mathrm{~min}$

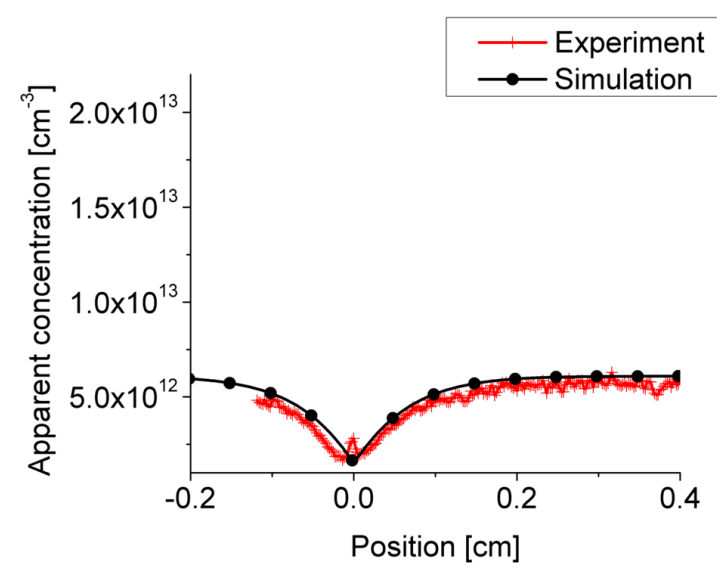

(b) $600^{\circ} \mathrm{C}$ for $160 \mathrm{~min}$

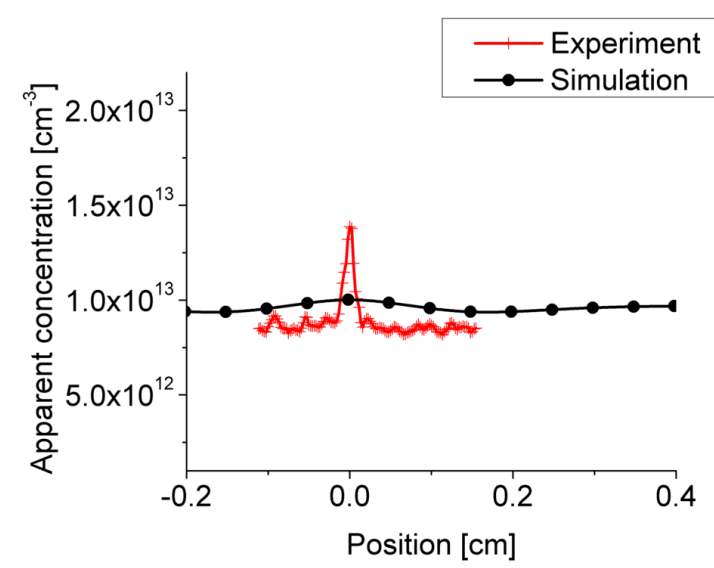

(d) $900^{\circ} \mathrm{C}$ for $60 \mathrm{~min}$

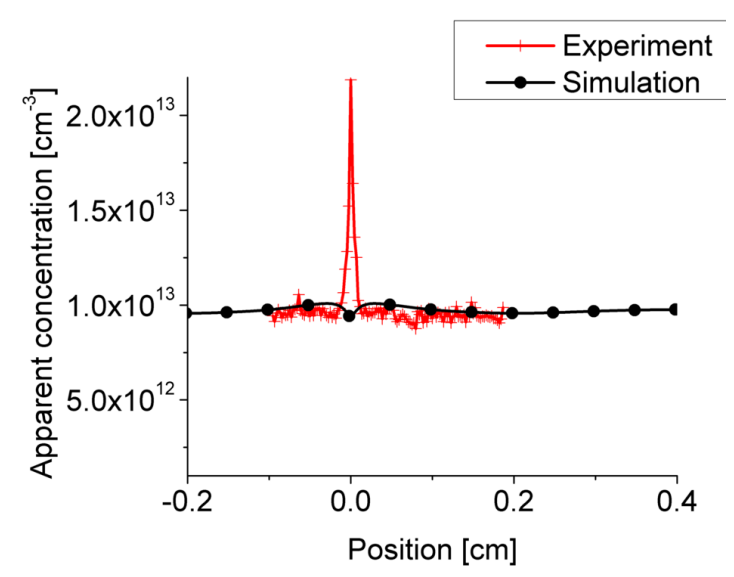

(f) $1000^{\circ} \mathrm{C}$ for $30 \mathrm{~min}$

Figure 40: Comparison of the line scans across the grain boundary for the modeled and measured apparent interstitial iron concentration for different annealing temperatures and annealing times. For the simulations the as-grown temperature curve from figure 24 was used for the as-cut state and the initial iron concentration was set to a lower value of $1 \cdot 10^{13} \mathrm{~cm}^{-3}$. 


\subsubsection{Conclusion from the comparison of LBIC and PL measurements}

The sections 5.3.1 to 5.3.3 have shown that there is good quantitative agreement of the simulations with the measured concentrations profiles, when assuming only precipitation as mechanism of accumulation at the grain boundary. The influence of segregation as mechanism of impurity accumulation was not included, but will be investigated by a simulation study in section 5.5 .

The region in the close vicinity of the grain boundary can not be reproduced by the simulations of the interstitial iron concentration. For the profiles of the LBIC measurements, it was shown that the peak in the vicinity of the grain boundary in the measurements can well be modeled by including the recombination activity of the grain boundary via an LBIC model. For the PL measurements it remains an open question if the peak in the vicinity of the grain boundary is a real concentration or if it is a measurement artifact originating from other recombination channels similar to the LBIC measurements [80, 102, 104]. There are strong indications from the comparison of simulations and LBIC measurements that the interstitial iron concentration is not dominating the recombination in the vicinity of the grain boundary. In this case other recombination channels can not be assumed to be negligible in the calculation of the lifetime from PL measurements as described in section 2.6 and in more detail by Macdonald et al. [40]. This suggests that the peak in the vicinity of the grain boundary in the concentration profiles calculated from PL measurement can not be interpreted as interstitial iron concentration.

Some discrepancies have been observed in the level of concentration at high temperature annealing between simulations and PL measurements. This could be due to an overestimation of the dissolution of precipitates during annealing. However, there is evidence that the discrepancies are due to the initial experimental conditions (temperature curve for the as-cut state, total iron content).

The comparison of the calculated diffusional ranges with the width of the denuded zone indicate that the precipitation at the grain boundary is mainly diffusion-limited for the temperature processes investigated here. It was illustrated that the denuded zone depends on temperature, cooling rate and on the initial state of the sample. To describe the influence of the temperature on the interstitial iron concentration profile, it is helpful to separate the annealing temperature $T$ into three regimes, depending on the equilibrium temperature $T_{e q}^{i}$ at which the interstitial iron concentration in the sample equals the solubility:

1. For $T<T_{e q}^{i}$ precipitation occurs. The final profile is influenced by the initial state of the sample in this case.

2. For $T \approx T_{e q}^{i}$ the interstitial iron concentration does not change significantly due to precipitation. In this temperature regime the influence of precipitate ripening becomes very important. Measured concentration profiles can not be reproduced very well in this regime because ripening is not included in the model.

3. For $T>T_{e q}^{i}$ dissolution of precipitates occurs. If the annealing temperature $T$ is also 
higher than the temperature $T_{e q}^{\text {tot }}$ at which the total iron concentration in the sample equals the solubility and the annealing time is sufficiently long, all precipitates are dissolved and a homogeneous concentration profiles is established equaling the total iron concentration. In this case the effective precipitate density depends on the supersaturation level and is strongly influenced by the cooling rate. If $T<T_{e q}^{t o t}$ (or the annealing time is not sufficiently long), some precipitates are remaining and the resulting profile is influenced by the initial state of the sample because precipitation occurs at already existing precipitates.

It has to be mentioned that the conversion from LBIC signal to apparent concentration via the diffusion length is not straight forward. For the calculation, there is a spread of values for the capture cross section $\sigma_{n}$ in the literature, ranging between orders of magnitude [105]. This affects the grain concentration level and the precipitate density at the grain boundary and in the grains, which are adjusted in the model to fit experimental results. With the conversion parameter experimentally determined by Istratov et al. [7] (see table 4 in section 4.3), fitting with reasonable values for the precipitate densities was not possible. Instead the value for the capture cross section $\sigma_{n}$ from Macdonald et al. [99] was used. Furthermore it is uncertain to which amount precipitates influence the measured diffusion length/ lifetime. Neglecting the contribution to the diffusion length from precipitates could be a reason why a conversion with the factor from Istratov et al. [7] led to an overestimation of the measured diffusion length. 


\subsection{Investigation of the influence of the parameters on the example of the as-grown simulation}

As section 5.3.1 has shown, the model with precipitation as mechanism of impurity accumulation at the grain boundary can reproduce measured apparent concentrations by choosing the right set of input parameters. In order to better understand the measured concentration profiles, it is now useful to perform simulations with variations of the different parameters. For all variations, the simulation with the parameters for the as-grown sample LBIC 1 from section 5.3.1 was used as reference.

\subsubsection{Variation of grain size}

The simulations in section 5.3.1 were done only for a grain size of $2 \mathrm{~mm}$ because the experimental line scans were extracted from a grain boundary adjacent to two grains with a grain size of approximately $2 \mathrm{~mm}$. However, multicrystalline silicon samples usually contain grains with a wide range of sizes. In order to receive more general statements and make a step towards a statistical analysis, in this section the grain size is varied and the influence of the grain size on the concentration profiles is investigated.

Figure 41(a) and figure 41(b) show profiles of the interstitial iron concentration and the concentration of precipitated iron, respectively, across a grain boundary. The simulations with a grain size of $2 \mathrm{~mm}$ (black circles in figures 41(a) and 41(b) corresponds to the as-grown simulation for sample LBIC 1 in section 5.3.1. The profiles show that for the interstitial iron concentration as well as for the concentration of precipitated iron in the grain the overall level of the profile in the grain and at the grain boundary and the depth of the denuded zone change with a variation of the grain size. Of course not only grain size, but also the total impurity concentration in the sample and the precipitate densities in the grain and at the grain boundary influence the level of the profile and the depth of the denuded zone. A variation of these three parameters will be described in the following sections 5.4.2, 5.4.3 and 5.4.4.

In figure 41(c) the concentration level at the maximum distance to the grain boundary of the profiles shown in figure 41(a) and 41(b), i.e. the 'grain level' of concentration, is plotted versus the grain size. Shown are the grain levels of the interstitial iron concentration, of the concentration of precipitated iron in the grain and of the total iron concentration, i.e. the sum of interstitial iron concentration and concentration of precipitated iron. The level for all concentrations increases with increasing grain size until it reaches a constant value for a grain size of approximately $5 \mathrm{~mm}$. Comparing this with the diffusional range of the impurities of $R_{m}=1.25 \mathrm{~mm}$, which is calculated by a numerical evaluation based on equation 3 in section 2.1 .2 assuming a total iron concentration of $5 \cdot 10^{13} \mathrm{~cm}^{-3}$, shows that for grains smaller than $5 \mathrm{~mm}$ the grain boundary lies within the diffusional range of the impurities, while for larger grains the maximum distance to the grain boundary is larger than the diffusional range of the impurities. A comparison of the minimum and maximum of interstitial iron concentration and concentration of precipitated iron in the grain in figure 41(c) shows that with increasing grain size, the increasing interstitial iron concentration results in a stronger precipitation in the grain. Therefore, the increase of interstitial 
iron concentration due to less influence of the grain boundary precipitation is compensated by stronger grain precipitation to some extent.

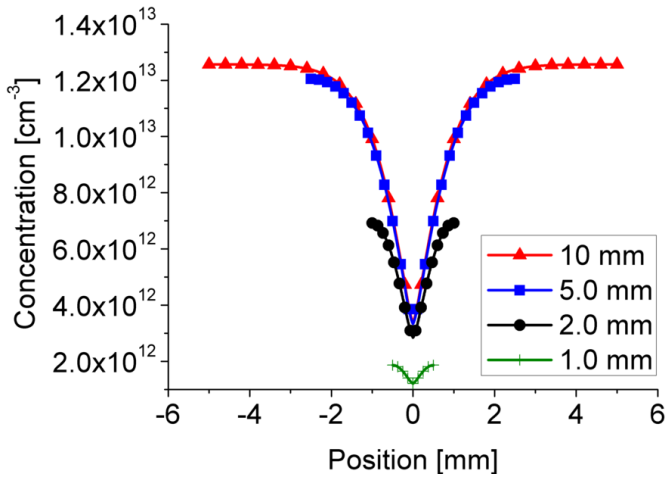

(a) Interstitial iron concentration

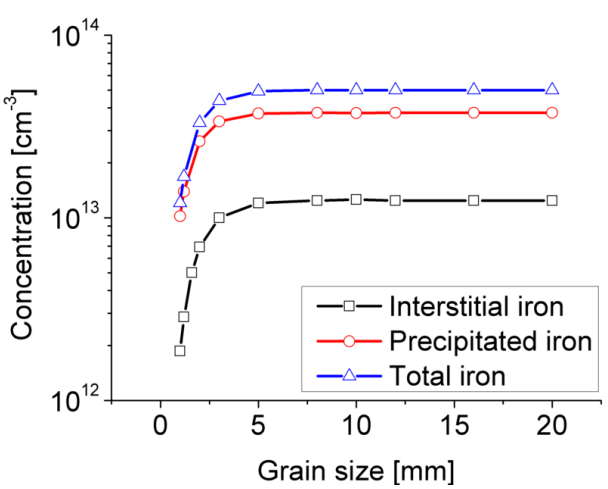

(c) Concentration level in the grain versus grain size

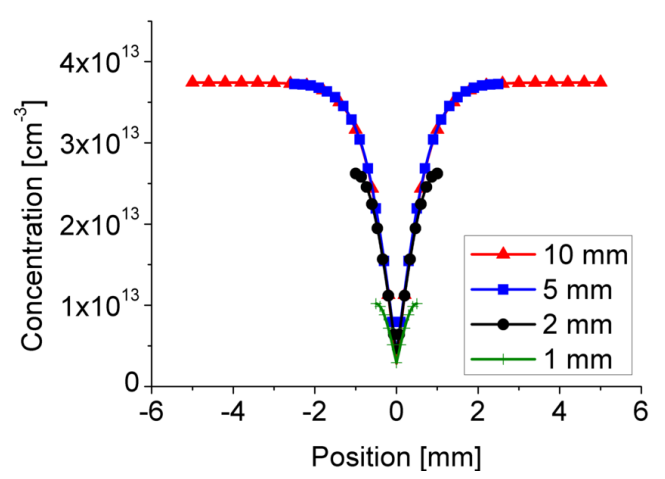

(b) Concentration of precipitated iron

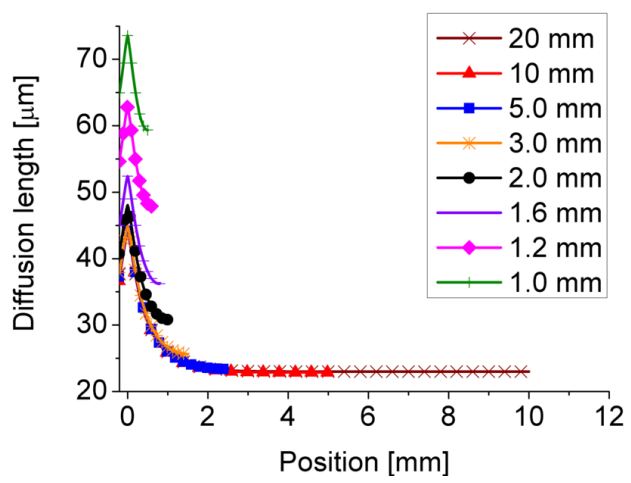

(d) Diffusion length for a variation of grain size

Figure 41: Variation of grain size for the as-grown simulation LBIC 1: figure 41(a) shows the profile of the interstitial iron concentration and figure 41(b) the profiles for the concentration of precipitated iron in the grain across the grain boundary. Figure 41(c) shows the concentration level in the grain versus grain size and illustrates that the concentration in the grain increases with increasing grain size until it reaches a constant level at a grain size of approximately $5 \mathrm{~mm}$. Figure 41(d) shows the corresponding diffusion length calculated from the interstitial iron concentration as described in section 2.4 .

Figure 41(d) shows the diffusion length profiles calculated from the interstitial iron concentration profiles in figure 41(a) via the Shockley-Read-Hall equation 6 in section 2.4 and with the parameters shown in section 4 . It shows a maximum of diffusion lengths at small distances to a grain boundary due to low interstitial iron concentrations in the denuded zones and low diffusion length at large distances to a grain boundary, especially for large grains.

These results can be compared to a typical histogram of the measured LBIC currents for the 
as-grown state as shown in figure 42(a), The figure is taken from Krenckel et al. [23]. To achieve this histogram a pattern recognition technique was used to sort local LBIC data according to the distance to the next grain boundary. The histogram shows the same trend for the measured photo currents as for the modeled diffusion length: The highest photo current is observed at small distances to the grain boundary, while for large distances to the grain boundary the photo current is low. Therefore, the measured photo currents and the modeled diffusion length are qualitatively in close accordance. However, the modeled diffusion length give additional information of the grain size.

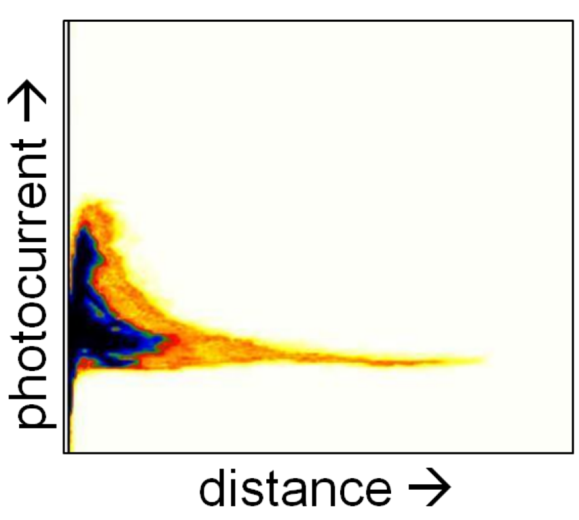

(a) Histogram

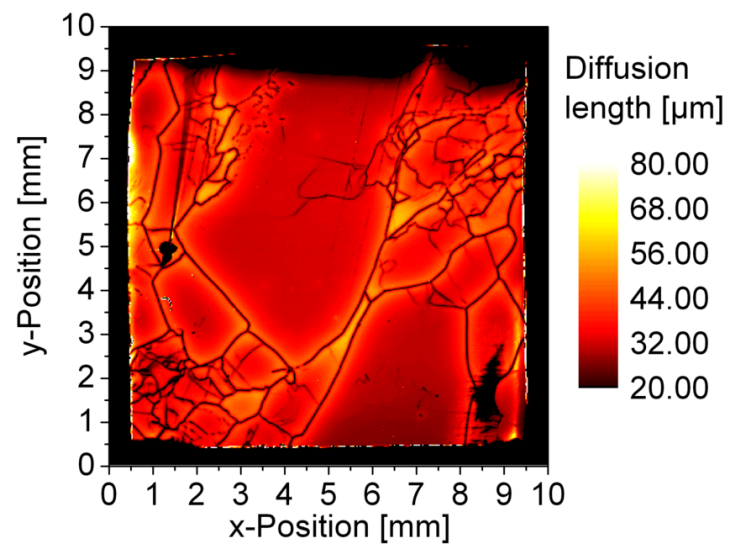

(b) Diffusion length map

Figure 42: Figure 42(a) shows the relative frequency histograms of the measured LBIC currents versus distance to the next grain boundary for the as-grown sample LBIC 1. It is taken from Krenckel et al. [23]. Figure 42(b) shows the diffusion length map calculated from the measured LBIC currents from the as-grown sample LBIC 1, from which the frequency histogram was calculated. The image was taken from Krenckel [42].

Figure 42(b) shows the two dimensional map of diffusion length calculated from the measured LBIC currents. The figure is taken from Krenckel [42]. A comparison with the modeled diffusion lengths in figure 41(d) shows that the modeled diffusion lengths are in the same range between $20 \mu \mathrm{m}$ and $80 \mu \mathrm{m}$ as the measured diffusion lengths. Both, the diffusion length map calculated from LBIC measurements in figure 42(b) and the modeled diffusion length profiles in figure 41(d) show a very important trend: smaller grains have a larger diffusion length. From simulations it can be deduced that this higher diffusion length is due to a lower interstitial iron concentration level in smaller grains for which the grain boundary lies in the diffusional range of the impurities. Therefore, an enhancement of diffusion length due to redistribution of impurities from the grain to the grain boundary by precipitation works better for small grain sizes. Taking the recombination losses due to the recombination of the contaminated grain boundaries into account, which are lower for larger grains (less grain boundaries), this implies that there is an optimal grain size to achieve optimal diffusion lengths. 


\subsubsection{Variation of total impurity concentration}

In this section the influence of the total iron concentration on the resulting interstitial iron concentration is investigated. As discussed in section 5.3.3 a different total iron concentration results in different concentration levels in the grain and different depth of the denuded zone. Therefore, it is important to understand the influence of the total iron concentration on the resulting interstitial iron concentration to appropriate model measured line scans.

Figure 43 shows results for the interstitial iron concentration for a variation of the total iron concentration. The legends gives the total iron concentration in $\mathrm{cm}^{-3}$.

The profile for a total iron concentration of $5 \cdot 10^{13} \mathrm{~cm}^{-3}$ (black line with circles) is the profile modeled to fit the as-grown sample LBIC 1 in section 5.3.1. In figure 43(a) it can be seen that for total iron concentrations $\geq 1 \cdot 10^{13} \mathrm{~cm}^{-3}$ the level within the grains decreases with increasing total iron concentration. Figure 43(b) shows that for low total iron concentration of $5 \cdot 10^{12} \mathrm{~cm}^{-3}$ only little and for a total iron concentration of $1 \cdot 10^{12} \mathrm{~cm}^{-3}$ negligible precipitation in the grain and at the grain boundary occurs. The variation of the resulting grain level of the interstitial iron concentration is illustrated in figure 43(c). It shows the value for the interstitial iron concentration at the maximum distance of $1 \mathrm{~mm}$ to the grain boundary, i.e. the level of interstitial iron concentration in the grain versus the total iron concentration. It reveals a peak in the grain level of the interstitial iron concentration around a total iron concentration of $2.5 \cdot 10^{13} \mathrm{~cm}^{-3}$. In this region of total iron concentration the concentration of precipitated iron becomes larger than the interstitial iron concentration and therefore significantly reduces the interstitial iron concentration. While for decreasing total iron concentrations the decrease of resulting interstitial iron concentration in the grain is due to the lower initial total iron concentration, the decrease of resulting interstitial iron concentration for increasing total iron concentration is caused by stronger precipitation. The normed interstitial iron concentration profiles in figure 43(d) illustrate the influence of a variation of total concentration on the denuded zone. It shows that the depth of the denuded zone increases with increasing total iron concentration due to stronger precipitation at the grain boundary. A stronger precipitation in the grain and at the grain boundary for higher initial iron concentrations has been observed in experiments [80, 77] and is interpreted as evidence for precipitation as mechanism of impurity accumulation. In order to illustrate the reasons, the increasing equilibrium temperature for the increasing total iron concentrations is shown in figure 44.

A higher equilibrium temperature results in an earlier onset of precipitation and therefore a higher concentration of precipitated iron in the grain and at the grain boundary due to a longer precipitation time. The stronger precipitation due to a larger precipitate size enhances the effect of the longer precipitation time. 


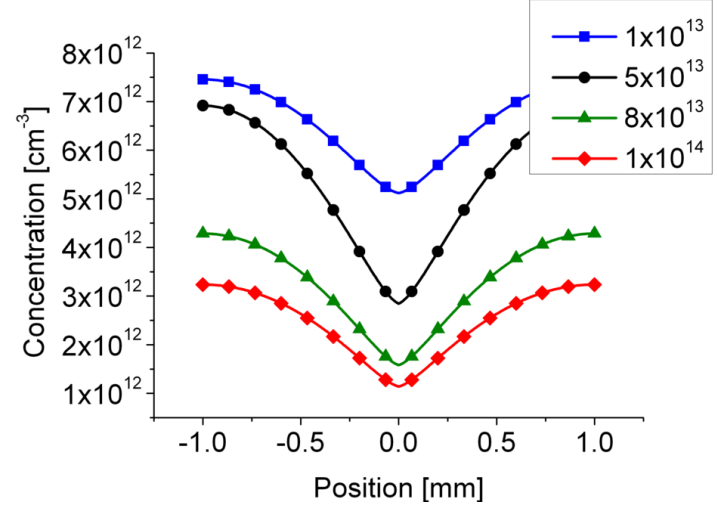

(a) High total iron concentrations

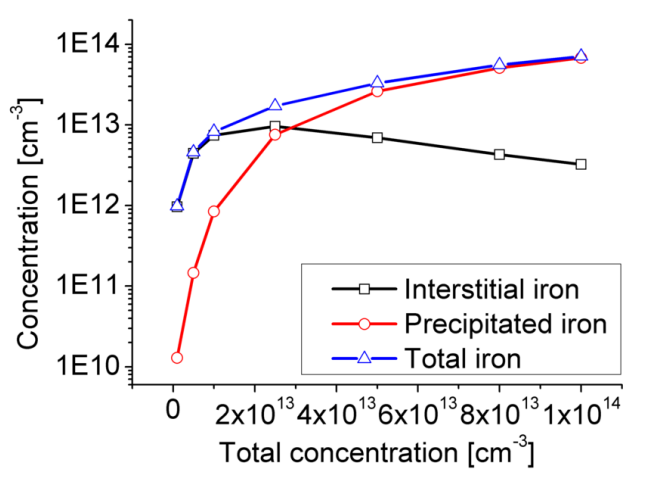

(c) Grain level vs. total iron concentration

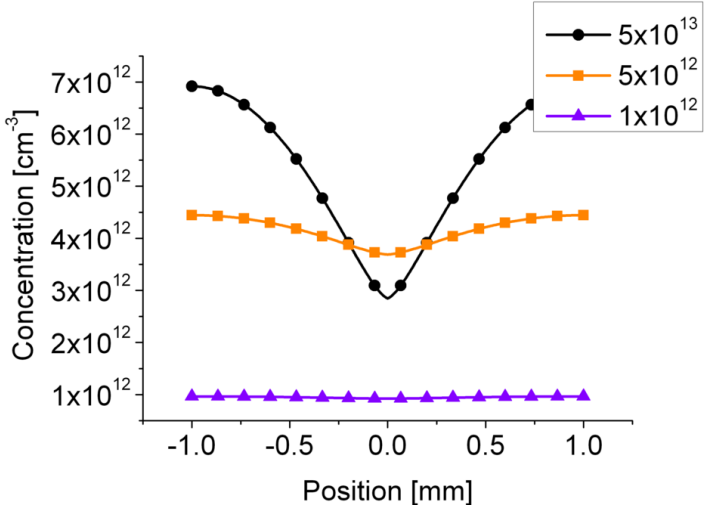

(b) Low total iron concentrations

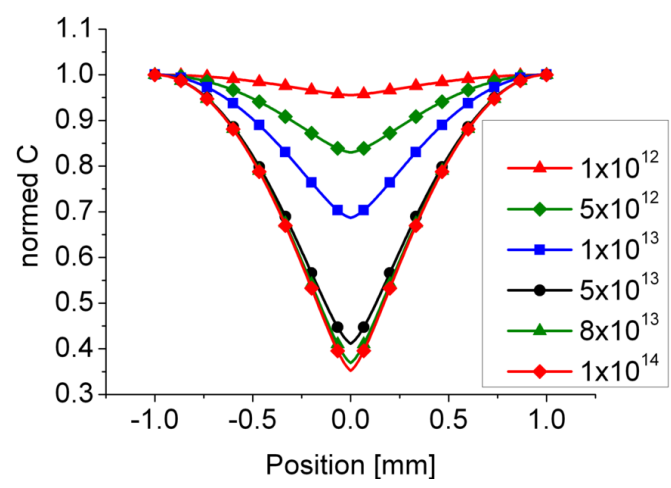

(d) Normed profiles for high and low iron concentrations

Figure 43: Results for a variation of total concentration for the as-grown sample LBIC 1 from section 5.3.1. The legend gives the total iron concentration in $\mathrm{cm}^{-3}$. Figures 43(a) and 43(b) show profiles of the interstitial iron concentration across the grain boundary for high and low total iron concentrations. The profile with a total iron concentration of $5 \cdot 10^{13} \mathrm{~cm}^{-3}$ corresponds to the reference simulation in section 5.3.1. Figure 43(c) shows the 'grain level' of the interstitial iron concentration at a position $x=1 \mathrm{~mm}$, i.e. in the grain at the maximum distance from the grain boundary versus total iron concentration. It illustrates that there is a maximum of resulting interstitial iron concentration at a total iron concentration of approximately $2.5 \cdot 10^{13} \mathrm{~cm}^{-3}$. For increasing and decreasing total iron concentration the resulting interstitial iron concentration decreases. The normed interstitial iron concentration in figure $43(\mathrm{~d})]$ shows the influence of a varying total iron concentration on the denuded zone. 


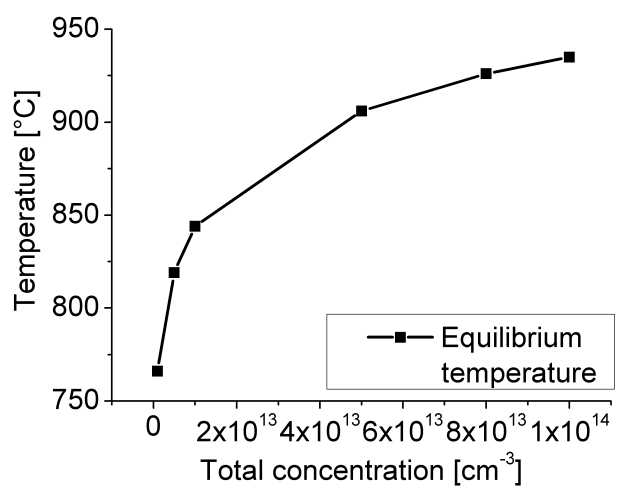

Figure 44: Equilibrium temperature for which the interstitial iron concentration equals the solubility and therefore precipitation starts for the various total iron concentrations. 


\subsubsection{Variation of precipitate density at the grain boundary}

The precipitate density at the grain boundary and in the grain influence the resulting interstitial iron concentration profiles as already discussed in section 5.3.1. In this section this influence will be investigated systematically.

Figures 45(a) and 45(b) show profiles of the interstitial iron concentration across the grain boundary for the as-grown sample LBIC 1 for a variation of precipitate density at the grain boundary for a large grain of $10 \mathrm{~mm}$ and a small grain of $2 \mathrm{~mm}$ respectively. The precipitate density in the legend is given in $\mathrm{cm}^{-2}$. The profile with a precipitate density at the grain boundary of $N_{g b}=1 \cdot 10^{7} \mathrm{~cm}^{-2}$ for a grain size of $2 \mathrm{~mm}$ (black circles in figure $45(\mathrm{~b})$ is the profile used to fit the line scan of the as-grown sample LBIC 1 in section 5.3.1. The profiles across the large grains shown in figure 45(a) illustrate that the depth of the denuded zone increases with increasing precipitate density. (The slightly lower level for a low precipitate density of $1 \cdot 10^{4} \mathrm{~cm}^{-2}$ at the grain boundary is due to an increased precipitation in the grain due to a higher interstitial iron concentration in the region of the denuded zone in comparison to large precipitate densities at the grain boundary.) From figure 45(b), it can be deduced that for small grains the precipitate density at the grain boundary influences the level of interstitial iron concentration within the grains and the interstitial iron concentration in the grain decreases with increasing precipitate density at the grain boundary. This result is in agreement with the results in section 5.4.1, in which the variation of grain size is investigated and it can be deduced that the precipitate density at the grain boundary influences the grain level of the interstitial iron concentration for grain sizes smaller than approximately $5 \mathrm{~mm}$, which approximately equals twice the diffusional range calculated for a total iron concentration of $5 \cdot 10^{13} \mathrm{~cm}^{-3}$ according to equation 3 in section 5.3 , The figure 45(b) shows that small grains in one sample can have very different interstitial iron concentrations due to different precipitation densities of the adjacent grain boundaries, even when the properties within the grain are equal. 


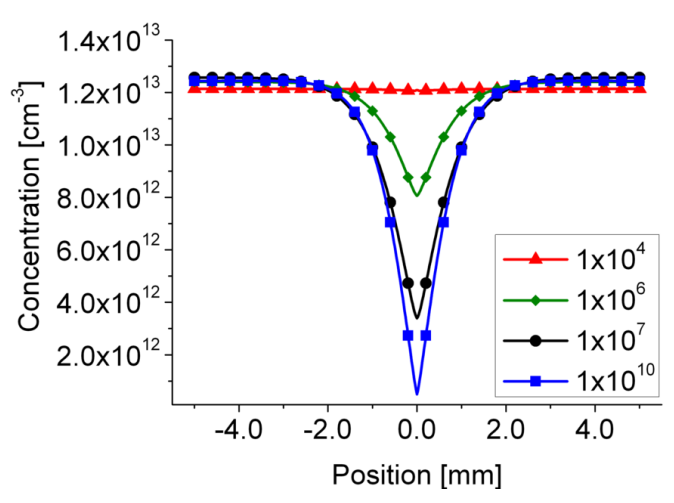

(a) Large $10 \mathrm{~mm}$ grain

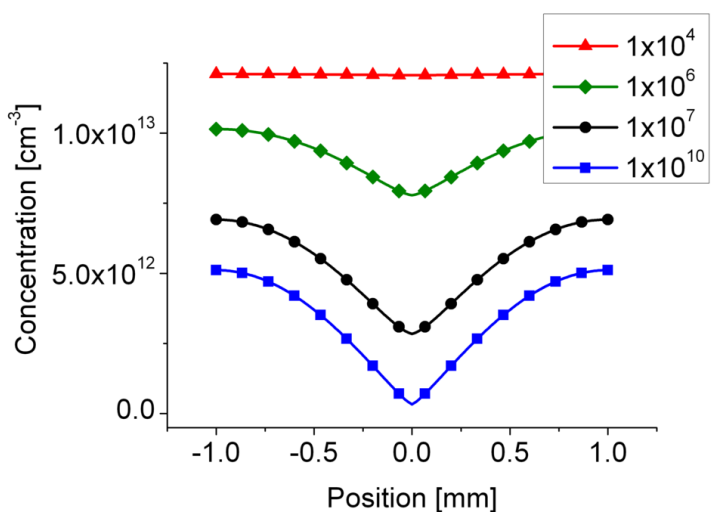

(b) Small $2 \mathrm{~mm}$ grain

Figure 45: Profiles of interstitial iron concentration across a grain boundary for a variation of precipitate density at the grain boundary for a large grain of $10 \mathrm{~mm}$ and a small grain of $2 \mathrm{~mm}$. The precipitate density is given in $\mathrm{cm}^{-2}$.

\subsubsection{Variation of precipitate density in the grain}

Figure 46 shows the influence of the precipitate density in the grain on the profile of the interstitial iron concentration across a grain boundary. The precipitate density in the grain is given in $\mathrm{cm}^{-3}$. The profile with a precipitate density in the grain of $N_{p}=1.5 \cdot 10^{7} \mathrm{~cm}^{-3}$ in figure 46(a) (black circles) is the profile used to fit the line scan of the as-grown sample LBIC 1 in section 5.3.1. Figure 46(a) shows the large influence of the variation of precipitate density in the grain on the overall level of the interstitial iron concentration. A higher density of precipitation sites results in a higher concentration of precipitated iron and therefore in a lower concentration of interstitial iron. The normed interstitial iron concentrations in figure 46(b) illustrate that the depth of the denuded zone decreases with increasing precipitate density in the grain due to the competition between precipitation at the grain boundary and in the grain: A higher flux of impurities towards the precipitates in the grain reduces faster the concentration of interstitial iron and results in a lower flux of impurities to the grain boundary.

The figures 45 and 46 show that there is a complex interplay between precipitate density in the grain and at the grain boundary. The depth of the denuded zone depends on the precipitate density in the grain and at the grain boundary and on their ratio. The level of interstitial iron concentration in the grain is influenced by the precipitate density in the grain. The precipitate density at the grain boundary influences the level of interstitial iron concentration for grains with a grain boundary within the diffusional range of the impurities. 


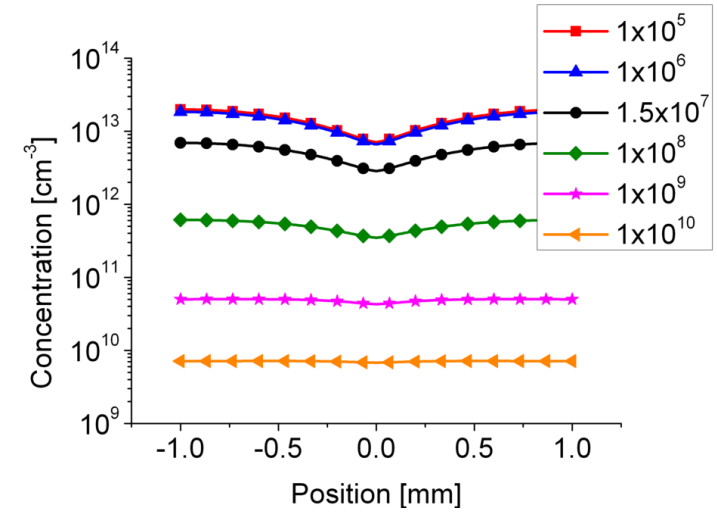

(a) Variation of precipitate density, logarithmic scale

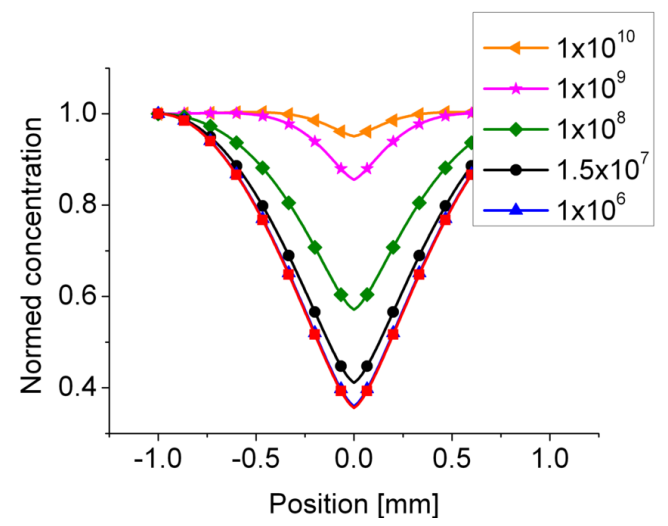

(b) Variation of precipitate density in the grain, linear scale

Figure 46: Variation of precipitate density in the grain. The legend gives the precipitate density in $\mathrm{cm}^{-3}$. Figure 46(a) shows the profile of interstitial iron concentration across the grain boundary in logarithmic scale and illustrates the decrease of the level of interstitial iron concentration with increasing precipitate density in the grain. The normed profiles in figure 46(b) show a decreasing depth of the denuded zone for increasing precipitate density in the grain. 


\subsection{Segregation versus precipitation}

As described in chapter 2.3, several mechanisms are proposed for the accumulation of impurities at grain boundaries and dislocations. In the previous studies, only precipitation was assumed as mechanism of impurity accumulation at the grain boundary. In the following section segregation at the grain boundary will be included in the simulations and the influence of segregation on the concentration profiles across grain boundaries will be investigated under various conditions.

There are two major differences between precipitation and segregation:

1. The flux towards precipitates depends on the precipitate size. The smaller the precipitates the smaller is the flux towards the precipitates, i.e. the flux at the onset of precipitation is small and grows slowly. For segregation the accumulation starts instantaneously with a high flux toward the grain boundary.

2. Precipitation occurs due to a supersaturation of interstitial impurities in the silicon, while segregation occurs when the ratio of the concentration in the grain boundary and in the silicon matrix is unequal to the segregation coefficient.

The temperature curves in this simulation study are designed to use these differences to get distinguishable concentration profiles for segregation and precipitation. In order to use the difference described in 1.), a temperature ramp with a very rapid cooling is used. To use the difference described in 2.), the temperature during the simulation must not decrease below the equilibrium temperature $T_{e q}^{\text {tot }}$, i.e. the temperature at which the solubility equals the total iron concentration in the sample.

Figure 47 shows the typical temperature ramp used in this study. An annealing step at a temperature $T_{1}$ to establish the equilibrium is followed by rapid cooling to a lower temperature $T_{2}$ at which accumulation of impurities at a grain boundary occurs. A variation of the temperature $T_{2}$ is investigated. Please note that the rapid cooling from $T_{1}$ to $T_{2}$ is set to the time $t=0 \mathrm{~s}$. The sample geometry is chosen to $2 \mathrm{~mm} \times 180 \mu m$.

Figure 48 shows two dimensional maps of the interstitial iron concentration $300 \mathrm{~s}$ after cooling to temperature $T_{2}$ for a simulation with only precipitation $448(\mathrm{a})$ and a simulation with precipitation and segregation $48(\mathrm{~b})$ as mechanisms for the impurity accumulation at the grain boundary.

The maps are results of simulations in which the sample is annealed at $T_{1}=1018^{\circ} \mathrm{C}$ for $50 \mathrm{~min}$ and rapidly cooled to $T_{2}=900^{\circ} \mathrm{C}$. The total iron concentration is chosen to $1 \cdot 10^{14} \mathrm{~cm}^{-3}$. The equilibrium temperature at which the solubility of interstitial iron in silicon equals the total iron concentration is $T_{e q}^{t o t} \approx 940^{\circ} C$, i.e. $T_{1}>T_{e q}^{\text {tot }}$ and $T_{2}<T_{e q}^{t o t}$. The density of precipitates is $N_{p}=2 \cdot 10^{6} \mathrm{~cm}^{-3}$ in the grain and $N_{g b}=1 \cdot 10^{7} \mathrm{~cm}^{-2}$ at the grain boundary and the segregation coefficient $S_{g b}$ is chosen to match the experimentally determined segregation coefficient $S_{A l}^{*}$ for iron at the interface between silicon and an aluminum gettering layer according to Abdelbarey et al. [39] because the segregation coefficient of grain boundaries is not known. When comparing figure 48(a) and 48(b), it is notable that the overall level of the interstitial iron concentration in 


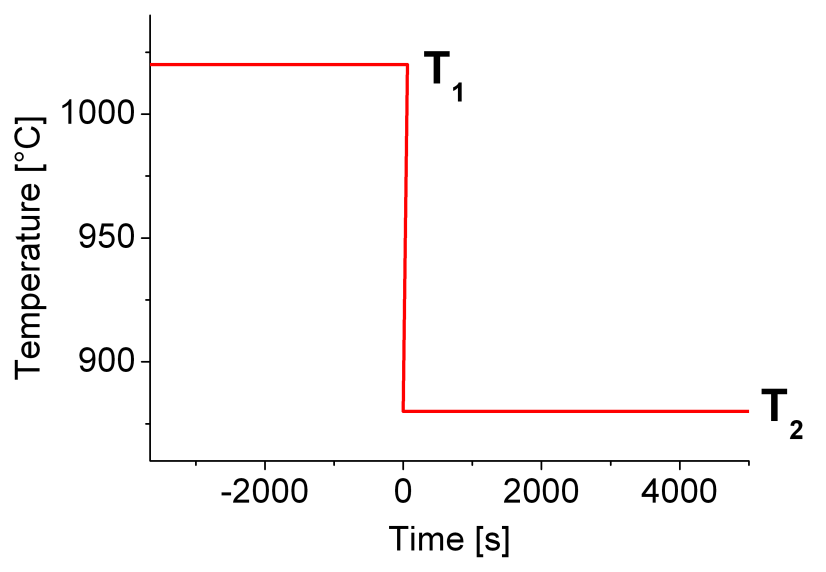

Figure 47: Typical temperature ramp for this simulation study. Please note that the rapid cooling from $T_{1}$ to $T_{2}$ is set to the time $t=0 s$.

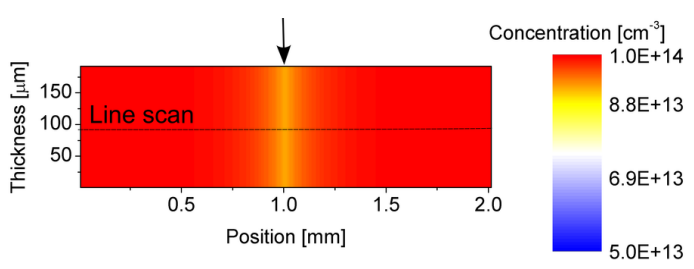

(a) Precipitation only

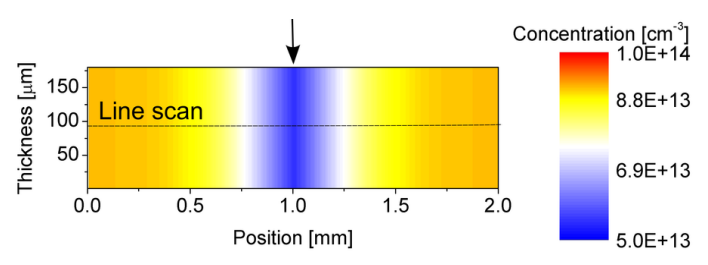

(b) Precipitation and segregation

Figure 48: Two dimensional map of the interstitial iron concentration $300 s$ after the rapid cooling for a simulation with the temperature ramp shown in figure 47. Figure 48(a) shows only precipitation and figure 48(b) shows a combination of precipitation and segregation as mechanisms of impurity accumulation at the grain boundary. The location of the grain boundary is indicated by the black arrows. 
the grain is lower for the combination of precipitation and segregation, in contrast to precipitation only. This is due to impurity segregation at the grain boundary during the annealing step while no precipitation occurs during annealing at $T_{1}$ because $T_{1}>T_{e q}^{\text {tot }}$. This is due to the different driving forces of precipitation and segregation described in 2.), i.e. at the temperature $T_{1}$ the ratio of concentrations at the grain boundary and in the silicon matrix does not equal the segregation coefficient $S_{g b}$, while there is no supersaturation of interstitial iron. The denuded zone (the region of decreased iron concentration in the vicinity of the grain boundary) is more distinct for the combined mechanisms of precipitation and segregation.

The dashed black line indicates the position of the line scans across the grain boundary shown in figure 49. Presented is the time evolution of the line scans for precipitation only (49(a)) and for a combination of precipitation and segregation $(49(\mathrm{~b})]$. A comparison of the two scenarios shows that for the simulation with segregation the denuded zone appears faster than for the simulation with only precipitation. This causes the different appearance in figure 48 . For longer times both line scans look very similar.

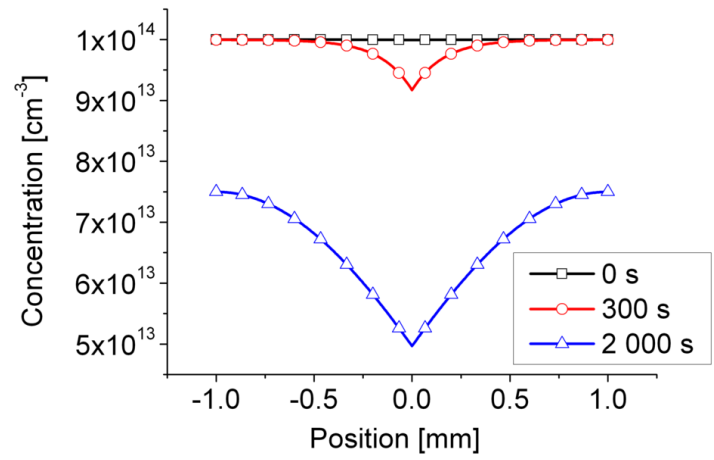

(a) Precipitation only

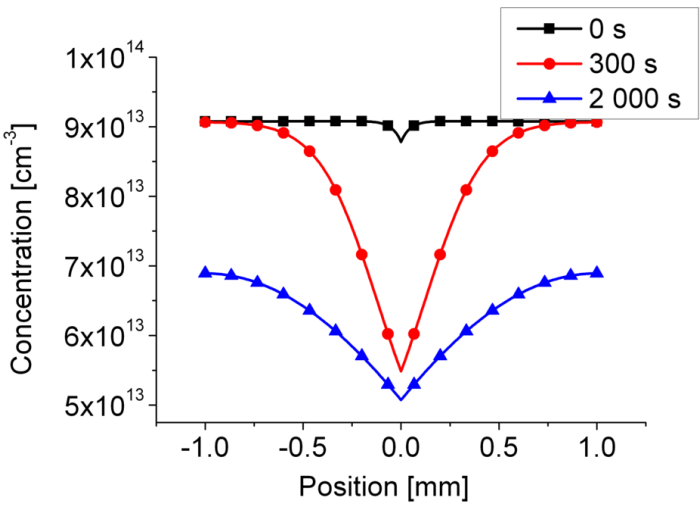

(b) Precipitation and segregation

Figure 49: Time evolution of a line scan of the interstitial iron concentration across the grain boundary for precipitation only and a combination of precipitation and segregation as mechanisms of impurity accumulation at the grain boundary. The line scans are extract at the position indicated by the black dashes lines in figure 48. It can be seen that the denuded zone develops faster for the simulation with segregation than for the simulation with only precipitation.

The different time scales for the formation of the denuded zone arise because the precipitates need some time to grow and the smaller the precipitates the smaller is the flux towards the precipitates at the grain boundary while the accumulation via segregation happens instantaneously as described in 1.).

Figure 50 shows interstitial iron concentration profiles for a simulation with changed $T_{2}$ and changed total iron concentration in order to investigate a scenario with $T_{2}>T_{e q}^{\text {tot }}$. The total iron concentration was set to $1 \cdot 10^{13} \mathrm{~cm}^{-3}$ and the temperature is changed to $T_{2}=880^{\circ} \mathrm{C}$. The equilibrium temperature for this simulation is $T_{e q}^{t o t} \approx 845^{\circ} \mathrm{C}$. Precipitate densities and segregation coefficient are kept as described in the simulations with $T_{2}<T_{e q}^{t o t}$. While the profiles for the simulation with only precipitation stay the same for all times, segregation causes accumulation 


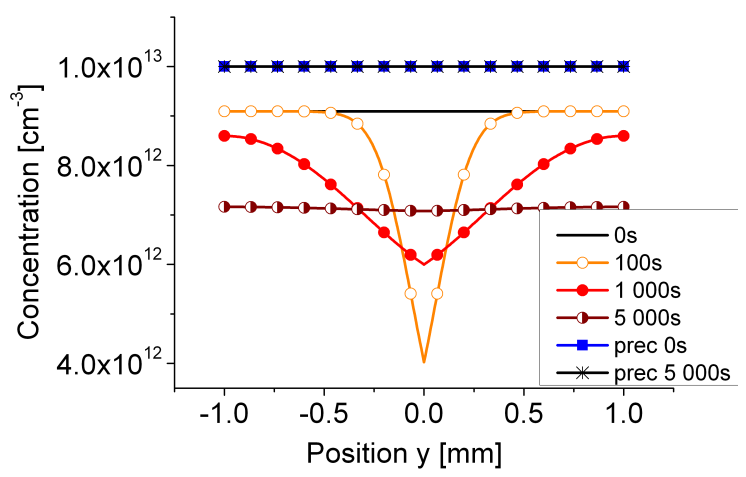

Figure 50: Interstitial iron concentration for a simulation with $T_{2}>T_{e q}^{\text {tot }}$ with precipitation only and a combination of precipitation and segregation. While the profile stays homogeneous for the simulation with only precipitation, a denuded zone develops for the simulation with segregation.

of impurities at the grain boundary and therefore a denuded zone in the line scan of the interstitial iron concentration.

In order to estimate, if the effects of segregation are measurable by LBIC, the diffusion length was calculated from the interstitial iron concentration according to the proposed proportionality constant from Istratov et al. [7] and the recombination effect was taken into account with the LBIC model as described in section 4.3. On the left of figure 51, the line scan of the diffusion length for the simulation with $T_{2}<T_{e q}^{\text {tot }}$ is shown for $300 \mathrm{~s}$. This is in the time range, where the difference between the simulation with precipitation and the combination of precipitation and segregation is largest. On the right of figure 51, the result for the simulation with $T_{2}>T_{e q}^{\text {tot }}$ is shown for $1000 s$.

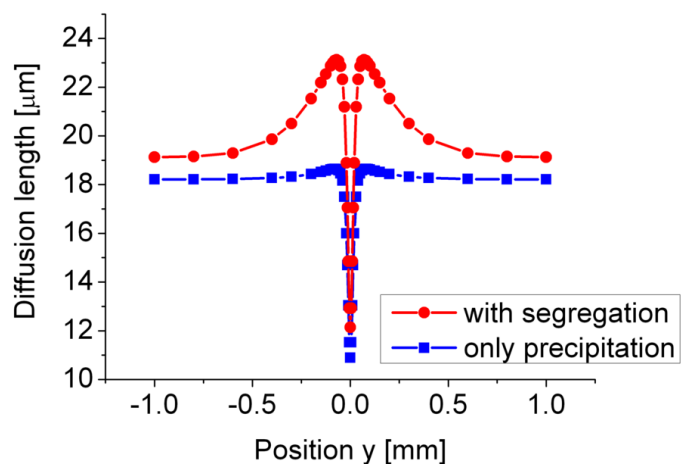

(a)

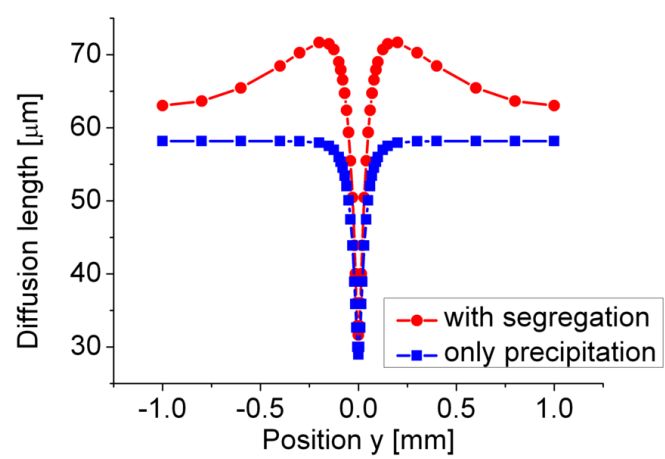

(b)

Figure 51: Diffusion length profile across the grain boundary after 300s for the simulation with $T_{2}<T_{e q}^{t o t}$ and after $1000 \mathrm{~s}$ for the simulation with $T_{2}>T_{e q}^{\text {tot }}$. The diffusion length is calculated from the modeled interstitial iron concentration and the effect of the recombination of the grain boundary is included. 
The profiles in figure 51 indicate that a difference would be measurable with LBIC for both scenarios. However, the precipitate density and the segregation coefficient of the grain boundary are usually not known, which makes it very difficult to determine the difference and the time in which a difference is visible because it both depends on the precipitate density and the segregation coefficient of the grain boundary.

The influence of the segregation coefficient is illustrated by figure 52, which shows interstitial iron concentration profiles for a variation of the segregation coefficient $S_{g b}=a \cdot \exp \left(\frac{b}{k T}\right)$ for the simulation with $T_{2}<T_{e q}^{\text {tot }}$ for 300s (see figure 52(a)) and 2000s (see figure 52(b)p. Varied was only the prefactor $a$ of the segregation coefficient. The exponential factor is set to $b=1.29 \mathrm{eV}$. This value for $b$ and a prefactor $a=1.87$ are the values determined by Abdelbarey et al. [39] for aluminum gettering and were used in the previous simulations.

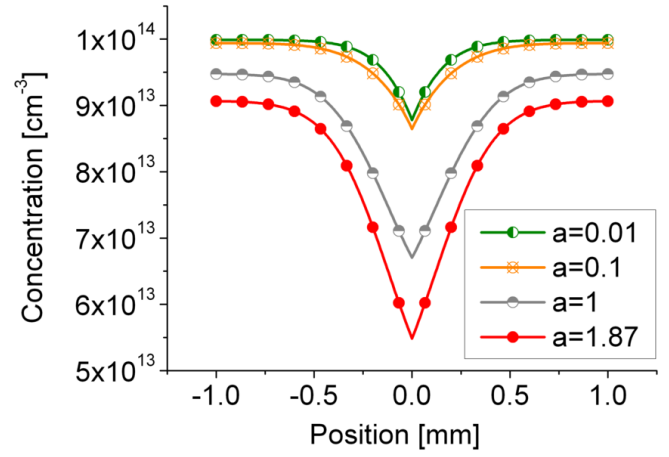

(a) Profiles after $300 \mathrm{~s}$

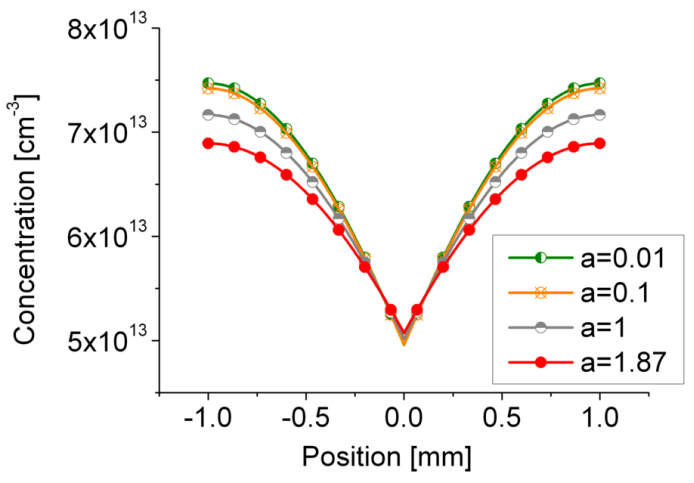

(b) Profiles after $2000 \mathrm{~s}$

Figure 52: Interstitial iron concentration profiles for a variation of prefactor $a$ of the segregation coefficient $S_{g b}=a \cdot \exp \left(\frac{b}{k T}\right)$ for segregation at the grain boundary. The exponential factor is held constant at $b=1.29 \mathrm{eV}$. This value for $b$ and a prefactor $a=1.87$ are the values determined by Abdelbarey et al. [39] for aluminum gettering which were used in the previous simulations. For decreasing segregation coefficient, the depth of the denuded zone decreases and the level of intersititial iron increases.

Both figures 52(a) and 52(b) show that with decreasing segregation coefficient the depth of the denuded zone decreases and the level of the interstitial iron concentration increases. The profiles for the prefactors of $a=0.1$ and $a=0.01$ are very similar and are not distinguishable from the profile for only precipitation in figure 49(a).

Figure 53 shows the concentration of precipitated impurities at the grain boundary and in the grain and the segregated impurities at the grain boundary versus time. It shows that the onset of precipitation is slow and increases over time, while segregation starts instantaneously and decreases over time. The graphs for the concentration of segregated impurities at the grain boundary do not start at the set minimum concentration because segregation already occurs during annealing in contrast to precipitation. It also shows that the concentration of precipitated 
impurities in the grain and at the grain boundary increases while the concentration of segregated impurities decreases with decreasing segregation coefficient. From this it can be deduced that the lower level of the interstitial iron concentration in the grains for high segregation coefficients is due to segregation at the grain boundary.

A similar effect has a variation of precipitate density at the grain boundary. As can be deduced from figure $45(\mathrm{~b})$ in section 5.4.3, a variation of precipitate density at the grain boundary can result in a change of the level of interstitial iron concentration in the grain and influences the shape of the denuded zone.

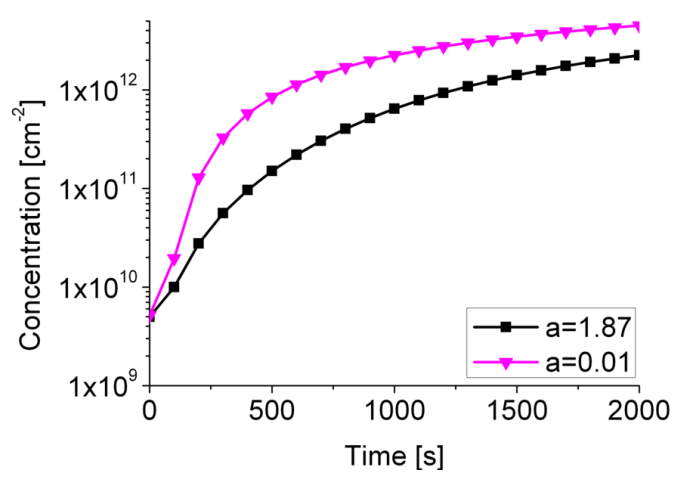

(a) Concentration of precipitated impurities at the grain boundary

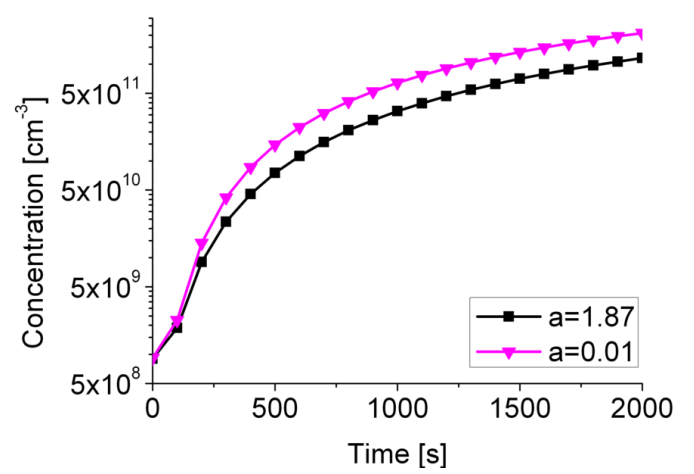

(c) Concentration of precipitated impurities in the grain

Figure 53: Concentration of precipitated and segregated impurities over time for two different prefactors $a=1.87$ and $a=0.01$ of the segregation coefficient $S_{g b}$. While the onset of precipitation is slow and the concentration of precipitated iron increases slowly over time, segregation starts instantaneously.

The foregoing observations indicate that an experiment according to the simulations with $T_{2}<T_{e q}^{\text {tot }}$ is not appropriate to distinguish between segregation and precipitation as mechanisms 
for impurity accumulation at grain boundaries because segregation coefficient and precipitate density at a grain boundary are usually not known. The simulations for $T_{2}>T_{e q}^{\text {tot }}$ indicate a measurable difference for a significant segregation independently from the precipitate density at the grain boundary.

We propose an experiment with a transition from $T_{2}<T_{e q}^{\text {tot }}$ to $T_{2}>T_{e q}^{\text {tot }}$ along a grain boundary to have a region where only precipitation as mechanisms for impurity accumulation is active and a region with a combination of precipitation and segregation as mechanisms for impurity accumulation. This can be achieved by a gradient in iron concentration along the grain boundary since $T_{e q}^{\text {tot }}$ changes with the interstitial iron concentration. The temperature $T_{2}$ can then be chosen to have a transition from $T_{2}<T_{e q}^{\text {tot }}$ to $T_{2}>T_{e q}^{\text {tot }}$ along the grain boundary. In the simulation presented in the following text, the concentration gradient along the grain boundary is achieved by an in-diffusion of iron at temperature $T_{1}=930^{\circ} \mathrm{C}$ for $1000 \mathrm{~s}$ with subsequent rapid cooling. It follows a temperature step of $T_{2}=880^{\circ} \mathrm{C}$ for $500 \mathrm{~s}$ without iron in-diffusion to cause precipitation and segregation of impurities at the grain boundary.

Figure 54 shows the resulting two dimensional interstitial iron concentration maps after the temperature step of $500 \mathrm{~s}$ at $T_{2}=880^{\circ} \mathrm{C}$ for a simulation with only precipitation (figure 54(a)) and a combination of segregation and precipitation as mechanisms for impurity accumulation at the grain boundary with three different prefactors of the segregation coefficient $a=1, a=0.1$ and $a=0.01$ (figures 54(b), 54(c), 54(d)). The exponential factor for the segregation coefficient is chosen to $b=1.29[\mathrm{eV}]$. Table 8 gives the values for the segregation coefficient $S_{g b}$ at $880^{\circ} \mathrm{C}$ for the different prefactors and relates it to the segregation coefficient $S_{A l}^{*}$ for aluminum gettering reported by Abdelbarey et al. [39]. The difference between the concentration for only precipitation and for a combination of precipitation and segregation decreases for decreasing prefactor of the segregation coefficient a. For a prefactor of $a=1$, as shown in figure 54(b), the maximum difference in concentrations amounts approximately $8 \cdot 10^{12} \mathrm{~cm}^{-3}$, while for a prefactor of $a=0.1$ in figure 54(c) the maximum difference is $1 \cdot 10^{12} \mathrm{~cm}^{-3}$ and for a prefactor of $a=0.01$ in figure 54(d) the maximum difference is only $8 \cdot 10^{11} \mathrm{~cm}^{-3}$. The maximum difference is at different locations in the sample for the three different prefactors and is not always located in the region $C_{i}<C_{e q}$.

\begin{tabular}{lll}
\hline \hline Prefactor $a$ & Segregation coefficient $S_{g b}$ at $800^{\circ} \mathrm{C}$ & $\frac{S_{g b}}{S_{A l}^{*}}$ at $800^{\circ} \mathrm{C}$ \\
\hline 1 & $4 \cdot 10^{5}$ & 0.5 \\
0.1 & $4 \cdot 10^{4}$ & 0.05 \\
0.01 & $4 \cdot 10^{3}$ & 0.005
\end{tabular}

Table 8: Segregation coefficients at $880^{\circ} \mathrm{C}$ for different prefactors. 


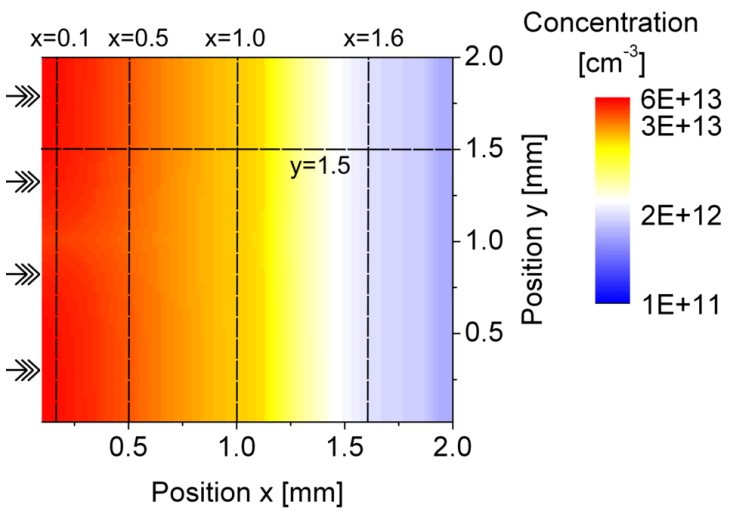

(a) Precipitation only

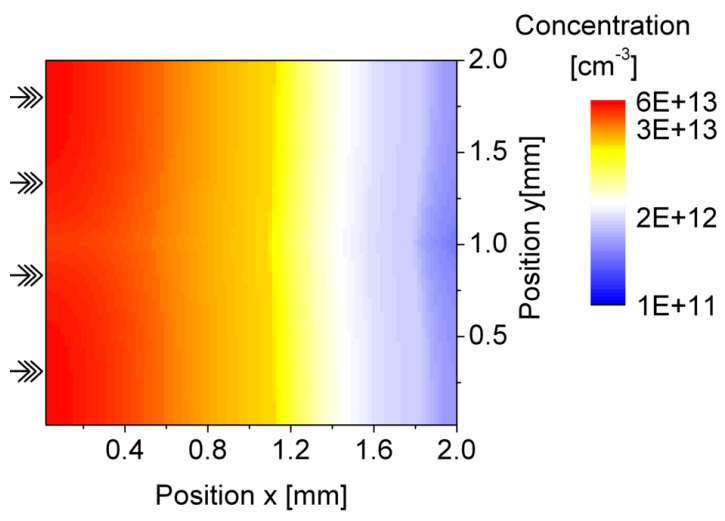

(c) Precipitation and segregation, $\mathrm{a}=0.1$

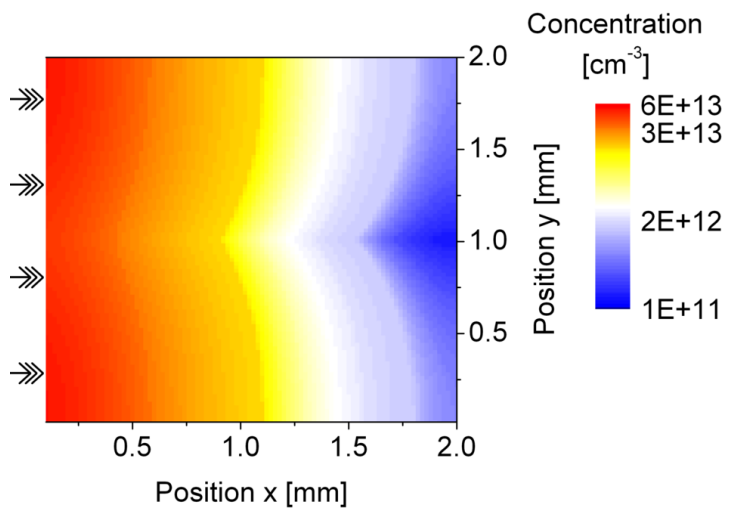

(b) Precipitation and segregation, $\mathrm{a}=1$

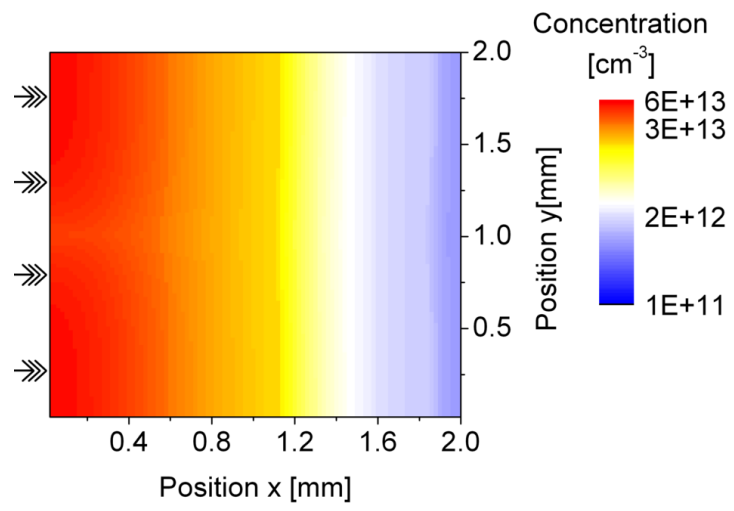

(d) Precipitation and segregation, $\mathrm{a}=0.01$

Figure 54: Interstitial iron concentration as a result of an iron in-diffusion simulation for precipitation and a combination of precipitation and segregation as mechanisms for impurity accumulation at the grain boundary for three different prefactors of the segregation coefficient: $a=1$, $a=0.1$ and $a=0.01$. The iron in-diffusion is pictured by the arrows on the left side. The dashed lines indicate the positions, where profiles were extracted. 
Figure 55 shows the gradient of interstitial iron concentration which is established after $1000 \mathrm{~s}$ of iron in-diffusion at $T_{1}=930^{\circ} \mathrm{C}$ and $500 \mathrm{~s}$ after the second temperature step at $T_{2}=880^{\circ} \mathrm{C}$. Shown are line scans extracted at a position $y=1.5 \mathrm{~mm}$ for precipitation only in figure 55(a) and for the combination of precipitation and segregation in figure $55(\mathrm{~b})$. The figures show that at this distance from the grain boundary, the mechanism of grain boundary accumulation has only little influence on the concentration gradient along the grain boundary. The temperature $T_{2}=880^{\circ} \mathrm{C}$ is chosen because the solubility of iron in silicon at this temperature is $C_{e q}=2.58 \mathrm{e}^{2} 3 \mathrm{~cm}^{-3}$ and lies well between the minimum and maximum interstitial iron concentration $C_{i}$ in the sample as can be seen from the interstitial iron concentration profiles in figure 55. The position where the interstitial iron concentration equals the solubility separates the sample in two regions with precipitation and segregation being active for $C_{i}>C_{e q}$ and only segregation being active for $C_{i}<C_{e q}$.

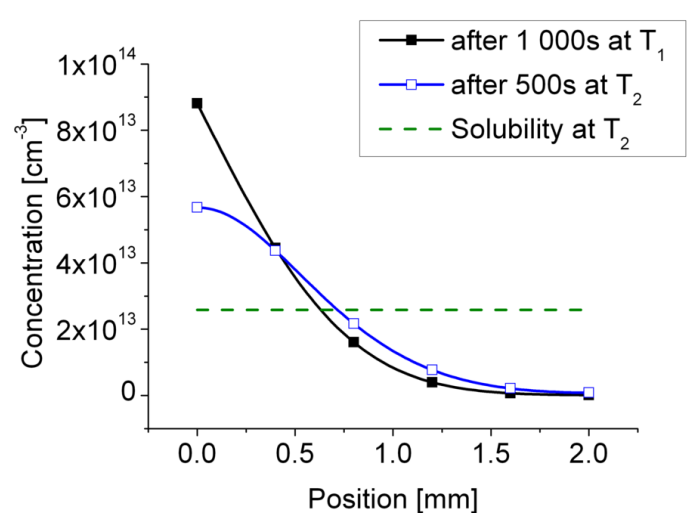

(a) Precipitation only

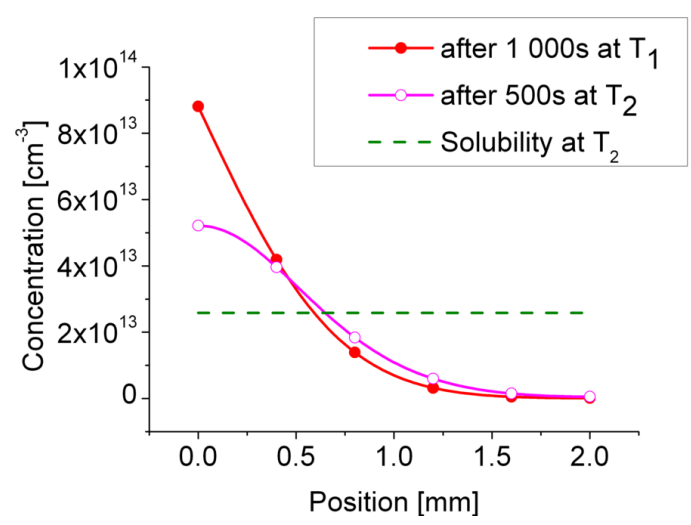

(b) Precipitation and segregation

Figure 55: Interstitial iron concentration profiles along the grain boundary at a position $y=$ $1.5 \mathrm{~mm}$ to show the concentration gradient established after $1000 \mathrm{~s}$ of iron in-diffusion at $T_{1}=$ $930^{\circ} \mathrm{C}$ and $500 \mathrm{~s}$ after rapid cooling to $T_{2}=880^{\circ} \mathrm{C}$. The dashed line indicates the value of the solubility of iron in silicon at the temperature $T_{2}$.

Figure 56 shows interstitial iron concentration profiles across the grain boundary for different $\mathrm{x}$-positions indicated by the dashed lines in figure 54. The comparison of figure 56(a) and 56(b) shows that for positions $x=0.1$ and $x=0.5$ the profiles for precipitation only and a combination of segregation and precipitation with a prefactor $a=1$ for the segregation coefficient look similar. For the positions $x=1$ and $x=1.6$ the profiles with segregation show a denuded zone in contrast to the profiles with precipitation only. The transition from $C_{i}>C_{e q}$, where precipitation occurs, and $C_{i}<C_{e q}$, where only segregation occurs, lies between the positions $x=0.5$ and $x=1$ as can be deduced from figure 55 .

The followging conclusions can be drawn from this section. As long as the range of the segregation coefficient for grain boundaries is unknown, simulations can not give a reliable prediction if segregation at the grain boundary has a measurable influence in an experiment according to the above presented simulations. A general problem when trying to develop an experiment to 


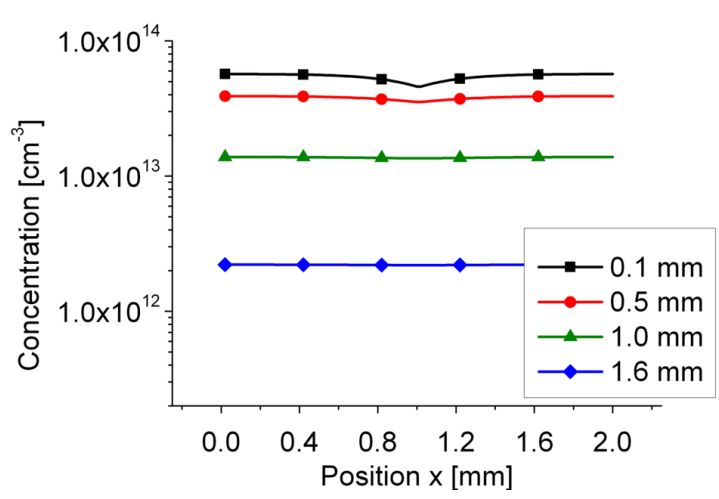

(a) Precipitation only

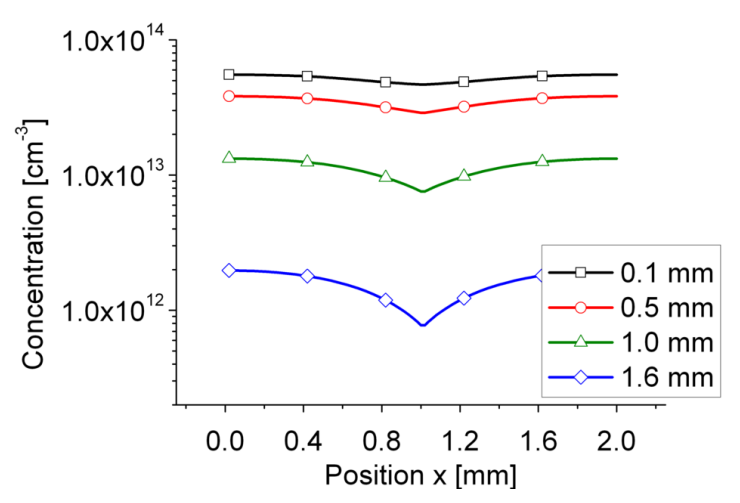

(b) Precipitation and segregation, $a=1$

Figure 56: Interstitial iron concentration profiles across the grain boundary at different $\mathrm{x}$ positions.

distinguish precipitation and segregation is that for high concentrations the difference is large, but there is no qualitative difference in the profiles. For regions in which the profiles show a qualitative difference, the concentrations are typically low giving a low concentration difference which is difficult to measure.

However, the comparison of experimental results and simulations promises to give some more insight into the order of magnitude of the segregation coefficient and to clarify if segregation has to be taken into account as mechanism of impurity accumulation at grain boundaries. Simulations give a first hint that segregation has only to be taken into account for special cases, while in most cases precipitation is expected to be the dominating mechanism and segregation can be excluded from the simulations. This means that in general it is appropriate to only include precipitation as mechanism for impurity accumulation at a grain boundary in the simulations, as has been done in this work. Conditions for which it might be necessary to take segregation into account are low precipitate densities at the grain boundary and temperature treatments at low temperatures and low total concentrations, especially when the interstitial iron concentration lies below the solubility. 


\section{Summary and outlook}

In this work the redistribution of metal impurities in multicrystalline silicon was explored. For this puropse single recombination active extended defects were investigated in detail with a combination of in-situ EBIC, FIB and TEM. Simulation studies were performed to investigate the influence of precipitates and accumulated impurities at grain boundaries on the distribution of metal impurities during gettering and other temperature processes. For the simulations, a metal impurity with the properties of iron was used. A comparison with experimental results from LBIC and PL measurements verified the underlying physical model of the simulations.

The method of combining in-situ EBIC, FIB and TEM, developed in this work, makes it possible to detect extended recombination active defect in silicon samples and investigate their distribution, atomic structure and chemical nature at high spatial resolution and low defect density. In section 3, it was shown that precipitates of metal impurities (here: copper) can be found at grain boundaries and dislocations and cause extended recombination active defects. The two investigated examples show that light elements like nitrogen and oxygen at grain boundaries are involved and serve as nucleation sites.

Further investigations with the method of combining in-situ EBIC, FIB and TEM are necessary to give a statistically more substantiated statement. An interesting opportunity to link the mechanisms taking place on wafer-scale with detailed information on atomistic scale will be, to combine the in-situ EBIC, FIB and TEM method with LBIC measurements (or other techniques to determine iron concentration on the the wafer-scale) and simulations.

The simulation study comparing phosphorus diffusion gettering and aluminum gettering in section 5.1 has shown that for both techniques, kinetics are limited by the dissolution of precipitates, if the gettering temperature lies below the equilibrium temperature at which the solubility equals the total iron concentration. This illustrates the need to investigate the influence of precipitates on the gettering kinetics. It was shown that for gettering temperatures which lie above the equilibrium temperature, aluminum gettering has the advantage of being only limited by the thermodynamic conditions determined by the segregation coefficient, while phosphorus diffusion gettering is limited by the phosphorus in-diffusion. As a consequence, aluminum gettering with its simpler underlying physics was chosen to be used for further investigations of impurity accumulation at grain boundaries. However, the use of aluminum gettering in practical solar cell processing is limited because the firing step is too short to have a significant gettering efficiency [16, 55].

In order to find optimal gettering processes, further simulation studies are necessary. For example, Plekhanov et al. [14] and Fenning et al. [100] propose the investigation of variable temperature processes with a high temperature step to dissolve precipitates and a subsequent low temperature step to make use of a high segregation coefficient.

The simulations of aluminum gettering with grid fingers in section 5.2.2 have shown that the gettering efficiency is much lower than for aluminum gettering with a continuous gettering layer. This is not only due to a lower segregation effect caused by less volume of the aluminum 
layer, but also due to kinetic reasons. While aluminum gettering with grid fingers is not appropriate to getter a high amount of impurities in a solar cell processing scheme, it results in interesting conditions for experiments of fundamental research because the simulations predict low concentrations of precipitated metal impurities, while the concentration of the interstitial metal impurities is still high. In comparison with a sample treated by gettering with a continuous gettering layer, this opens a possibility to study the influence of precipitated impurities e.g. on the diffusion length measured with LBIC or other recombination-sensitive techniques.

The simulations of aluminum gettering in presence of a grain boundary in section 5.2.3 illustrated that the grain boundary serves as source for interstitial impurities and causes recontamination of the grains. This re-contamination is most significant in the vicinity of the grain boundary, where it even influences the dissolution of the precipitated impurity in the grains. In the simulations, the precipitated impurities at the grain boundary dissolve significantly slower than the precipitates in the grains. It can be concluded that the dissolution of precipitates at the grain boundary influences the gettering kinetics significantly.

A prospective aim is a quantitative comparison of the modeled interstitial iron concentrations of gettered samples with experimentally determined interstitial iron concentrations, which does not succeed at present state. The most probable reason is that for low iron concentrations, as they occur in gettered samples, the interstitial iron concentration is not the limiting factor on lifetime/ diffusion length. Therefore the conversion from lifetime/ diffusion length to interstitial iron concentration is not valid and other recombination channels have to be taken into account. This has also been proposed by Schön et al. [25]. The origin of other recombination channels might be precipitates in the grains or other defects. The consideration of the precipitated iron as discussed in Cañizo and Luque [29] are a next possible step towards an improvement of the quantitative comparison of modeled and experimentally results for samples with low interstitial iron concentration.

While the quantitative comparison does not succeed for low interstitial iron concentrations, it works very well for samples with larger interstitial iron concentrations $\left(>1 \cdot 10^{12} \mathrm{~cm}^{-3}\right)$. This is shown in section 5.3, in which the accumulation of metal impurities at grain boundaries is simulated and compared with LBIC and PL measurements. For the simulation model, precipitation as the mechanism of impurity accumulation was used, while segregation was excluded. The good agreement of simulations and experiments indicates that precipitation is the dominating mechanism. This is consistent with the experimental findings of precipitated metal impurities at grain boundaries in section 3 .

The simulations showed that for some temperature treatments, it is important to take the history of temperature treatments of the sample into account because the resulting distribution of impurity concentrations is influenced not only by the precedent temperature treatment, but also by previous ones. Therefore, it is necessary to carefully consider the conditions of a temperature treatment. Especially it has to be examined if the solubility lies below or above the total iron concentration of the sample and if the annealing time is sufficient to dissolve all precipitates or if some precipitates are remaining in the sample.

The comparison of calculated diffusional ranges with the width of the denuded zones around 
grain boundaries give an indication that the width for the investigated concentration profiles is mainly limited by diffusion.

The comparison of simulations with LBIC measurements has shown that there is a region in the vicinity of the grain boundary, in which the LBIC current can not simply be interpreted as interstitial iron concentration. In this region the recombination activity of the grain boundary itself has to be taken into account. It is possible to model the effect of the recombination activity of the grain boundary, when including a charge carrier recombination velocity at the grain boundary and the laser conditions of the LBIC measurement. The quantitative agreement of the simulations and the measurements opens the way to quantitatively investigate the influence of impurity accumulation at grain boundaries on their recombination activity: from the width of the denuded zone around a grain boundary, it can be deduced how much impurities are accumulated at the grain boundary. This can be set into relation to the recombination activity of the grain boundary.

A discrepancy in the close vicinity of a grain boundary between the simulations and the PL measurements was observed, similar to the discrepancy for the LBIC measurements. It was proposed that this discrepancy originates from the same effect that the interstitial iron concentration is not the dominating recombination mechanism in the vicinity of the grain boundary and therefore other recombination channels have to be taken into account [40].

The comparison of PL measurements and simulations indicates that, for conditions at which precipitate ripening becomes an important process, the model is not accurate because it does not take ripening into account. In order to improve the model, it would be possible to include ripening via Fokker-Planck equations. However, solving of the Fokker-Planck equations during simulations is very time intensive and for most cases the recent model is sufficient to reproduce experimental results.

To clarify if discrepancies in the concentration level for high temperatures between simulations and PL measurements indicate an overestimation of dissolution of precipitates or are simply due to uncertain initial experimental conditions (temperature curve, total iron content), further comparison of simulations with measurements of well known experimental conditions are necessary.

As described in the conclusion in section 5.3.5, the calculation of the diffusion length in order to compare simulations with experiments is not straight forward. Since a quantitative agreement was not successful with the experimentally determined conversion factor from Istratov et al. [7], the capture cross section for FeB pairs from Macdonald et al. [99] were used. It has to be noted that there is a wide range of parameters available in literature which makes the conversion somewhat arbitrary. Furthermore, it is not clear if the recombination activity from precipitated impurities has to be taken into account. As already mentioned, the recombination activity of precipitated iron probably has to be taken into account for samples with low interstitial iron concentrations (e.g. after aluminum gettering). It is possible that it is not negligible for higher interstitial iron concentrations either. An experiment with grid finger gettering as proposed above might help to clarify this issue.

Recent experimental observations also indicate that for thin wafers $(\approx 150 \mu m)$ the recombination at the back side of the wafer have to be taken into account. 
The parameter variations of the simulation for an as-grown wafer in section 5.4 showed that smaller grains show a higher diffusion length (i.e. a lower interstitial iron concentration) than larger grains. The reason is that the grain boundary lies outside of the diffusional range of the impurities for large grains. Taking the recombination losses due to the recombination of the contaminated grain boundaries into account (less recombination losses for a lower number of grain boundaries), this implies that there is an optimal grain size in the range of $2 \cdot R_{M}$ to achieve optimal diffusion lengths. A comparison of simulations with experimental results showed a consistent picture. It was also shown in the simulations that the two processes of precipitation in the grains and precipitation at the grain boundary compete, i.e. the amount precipitated at the grain boundary/ in the grains depends not only on the initial concentrations and the precipitate density, but also on the ratio of precipitate density in the grain and at the grain boundary. Furthermore, it was shown that the variation of precipitate density at the grain boundary results in very different concentrations in the grain, especially for small grains. This indicates that grains with similar properties, but adjacent to different grain boundaries, can have very different diffusion length/ concentration levels.

A variation of the total initial iron concentration showed that higher initial iron concentration resulted in lower remaining interstitial iron concentrations, which is in accordance with experimental observations [80, 77].

The section 5.5 has shown that, at the current state, quantitative simulations of segregation as mechanism at the grain boundary are not possible because of lacking knowledge about the range of the segregation coefficient of grain boundaries. However, the simulations predict that segregation, in most cases, has only a minor effect while precipitation as mechanism of impurity accumulation at grain boundaries is the dominant mechanism. This is in agreement with the experimental results in section 3, in which only precipitated metal impurities at grain boundaries were detected and with the results in section 5.3 which showed that a model with only precipitation as mechanism of impurity accumulation can reproduce the experimental results. Simulations predict that segregation might be important for low precipitate densities at the grain boundary and temperature treatments at low temperatures with low total concentrations lying below the solubility of the interstitial metal impurity in silicon.

The comparison of simulations with specially designed experiments, such as the proposed iron in-diffusion experiment described in section 5.5, promise to contribute to an estimate of the range of the segregation coefficient and of the importance of segregation as mechanism of impurity accumulation.

In general similar simulations investigating the accumulation of impurities at grain boundaries for other metal impurities, e.g. copper, are of interest and easy to implement into the simulation model. However, while there are many measurements on iron in literature, other metal impurities are difficult to measure. Especially the fast diffusion copper can hardly be measured in its interstitial form due to its high mobility. An interesting issue for future simulations will be the investigation of lower precipitate densities at grain boundaries via single point sources located at the grain boundary. 


\section{Appendix}

\subsection{Mesh settings and boundary conditions for iron in-diffusion}

The boundary conditions for iron in-diffusion at the left boundary as described in section 4.2.1 are discontinuous, which causes numerical errors during simulations. In order to avoid these numerical problems the boundary conditions have to be smoothed. This is done by defining a smoothed Heaviside function and by refining the mesh at the left boundary, as shown in figure 57. For the refinement, an arithmetic sequence as mesh distributions with 1000 elements and an element ratio of 0.001 was used on the left boundary and a geometric sequences with 100 elements and an element ratio of 0.002 was used on the boundaries on the top and bottom. For details please refer to the Comsol manual [106].

Figure 58 shows a one-dimensional line scan of the two dimensional modeled resulting interstitial iron concentration profiles with and without smoothed Heaviside function and refined mesh for a temperature of $T=900^{\circ} \mathrm{C}$, an initial concentration at the left boundary of $C_{\text {init }}=10^{14} \mathrm{~cm}^{-3}$ and at an in-diffusion time of $t=10^{-5} \mathrm{~s}$. The mesh geometry is quadratic because sample thickness and size were scaled to 1 . (For details on scaling see section 4.2.6.) The original size of the sample is $1 \mathrm{~cm} \times 100 \mu \mathrm{m}$. It shows that the numerical error can have a large effect on the concentration profile at the side of the sample where the in-diffusion takes place. However, this is only true for very short in-diffusion times. For the present example the solutions for unrefined and refined mesh coincide already at an in-diffusion time of $t=0.1 \mathrm{~s}$. Nevertheless, it is important to keep in mind that the discontinuous boundary conditions bear numerical errors. 


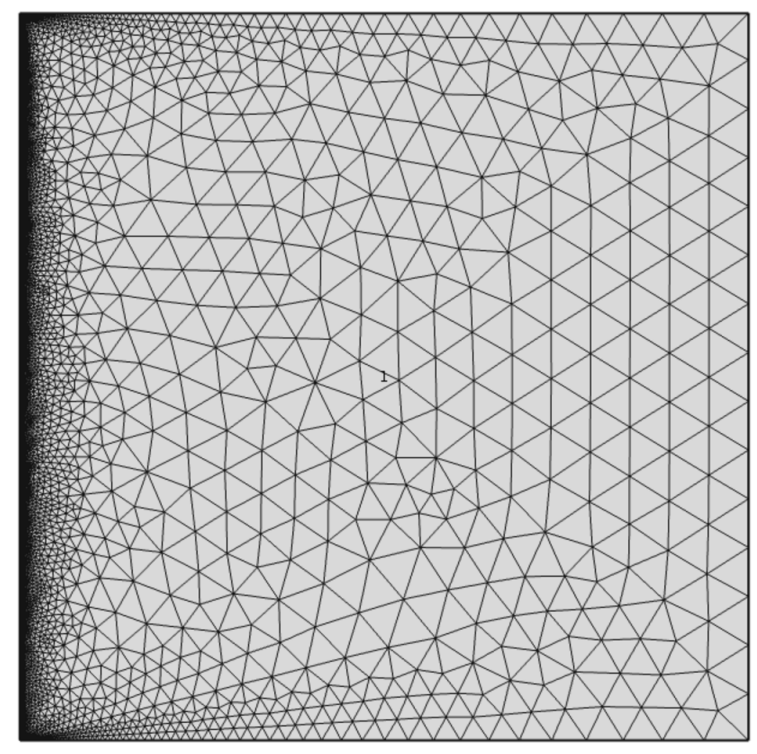

Figure 57: Refined triangular mesh to avoid numerical errors in in-diffusion zone (in-diffusion from left side). Shown is the non-dimensionalized geometry of a sample: thickness and width are scaled to 1 .

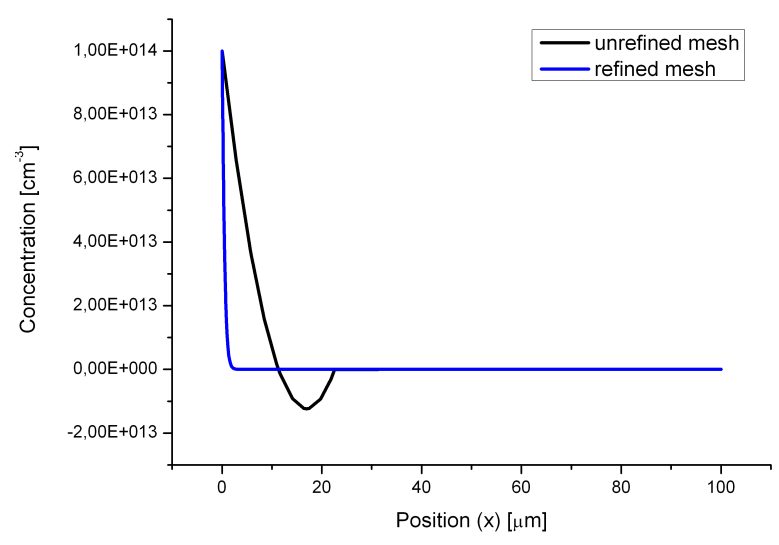

Figure 58: Comparison of the interstitial iron concentration profile with and without smoothed Heaviside function and by refining the mesh at the left boundary. 


\subsection{Mesh settings at the grain boundary}

In order to get a smooth solution for the concentration profiles of interstitial iron in silicon $C_{S i}$ and of precipitated iron in the grain $C_{p}$, the mesh has to be refined on the grain boundary. An example of an appropriate mesh refinement is shown in figure 59. It can be achieved by using a arithmetic sequence as mesh distributions with 80 elements and an element ratio of 0.01 . For details please refer to the Comsol manual [106]. As already described in section 7.1, the sample geometry was scaled and therefore the mesh geomtry is quadratic.

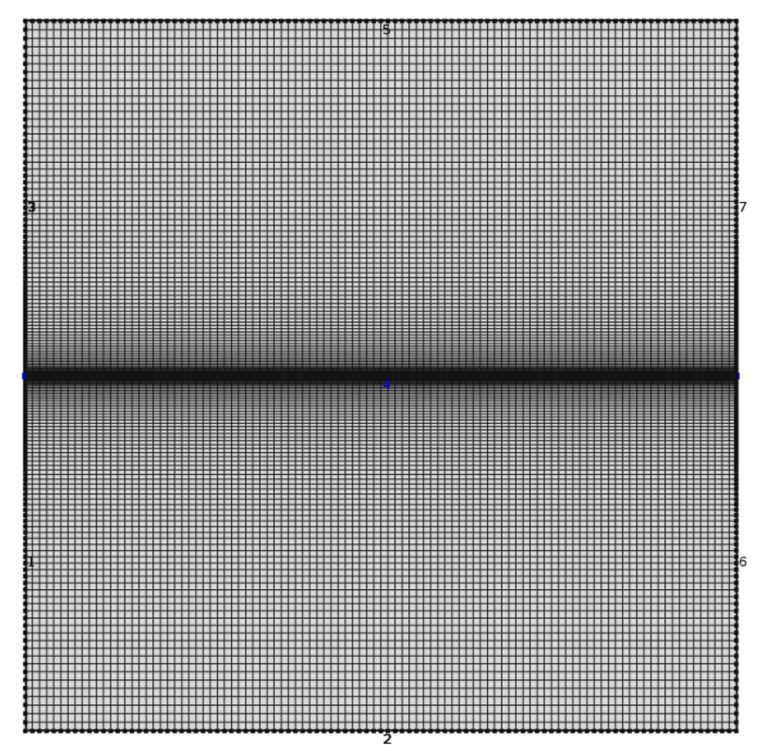

Figure 59: Refined rectangular mesh for a grain boundary as flux/source. Shown is the nondimensionalized geometry of a sample: thickness and width are scaled to 1 . The original size of the sample is $2 m m x 180 \mu m$. 


\section{References}

[1] A. Luque and S. Hegedus. Handbook of Photovoltaic Science and Engineering. John Wiley \& Sons Ltd. England, 2003.

[2] G. Masson, M. Latour, M. Rekinger, I. Theologitis, and M. Papoutsi. Global market outlook for photovoltaics 2013-2017. Technical report, European Photovoltaic Industry Association, May 2013.

[3] greentechsolar: S. Mehta. http://www.greentechmedia.com/articles/read/global-2013-pvmodule-production-hits-39.8-gw-yingli-leads-in-production-a, April 23, 2014. [Online; accessed 18-July-2014].

[4] J. Chen, T. Sekiguchi, and D. Yang. Electron-beam-induced current study of grain boundaries in multicrystalline Si. physica status solidi (c), 8:2908 - 2917, 2007.

[5] T. Buonassisi, A. A. Istratov, S. Peters, C. Ballif, J. Isenberg, S. Riepe, W. Warta, R. Schindler, G. Willeke, Z. Cai, B. Lai, and E. R. Weber. Impact of metal silicide precipitate dissolution during rapid thermal processing of multicrystalline silicon solar cells. Applied Physics Letters, 87:121918, 2005.

[6] D. Macdonald, A. Cuevas, A. Kinomura, Y. Nakano, and L. J. Geerligs. Transition-metal profiles in a multicrystalline silicon ingot. Journal of Applied Physics, 97:033523, 2005.

[7] A.A. Istratov, H. Hieslmair, and E.R. Weber. Iron contamination in silicon technology. Applied Physics A, 70:489-534, 2000.

[8] S. A. McHugo, H. Hieslmair, and E. R. Weber. Gettering of metallic impurities in photovoltaic silicon. Applied Physics A: Materials Science and Processing, 64:127-137, 1997.

[9] A. A. Istratov, T. Buonassisi, R. Schindler R. J. McDonaldand A. R. Smith, J. P. Kalejs J. A. R and, and E. R. Weber. Metal content of multicrystalline silicon for solar cells and its impact on minority carrier diffusion length. Journal of Applied Physics, 94:6552, 2003.

[10] T. Buonassisi, A. A. Istratov, M. D. Pickett, M. Heuer, J. P. Kalejs, G. Hahn, M. A. Marcus, B. Lai, Z. Cai, S. M. Heald, T. F. Ciszek, R. F. Clark, D. W. Cunningham, A. M. Gabor, R. Jonczyk, S. Narayanan, E. Sauar, and E. R. Weber. Chemical natures and distributions of metal impurities in multicrystalline silicon materials. Progress in Photovoltaics: Research and Applications, 14:513-531, 2006.

[11] C. Rudolf. Transmissionselektronenmikroskopische Untersuchungen zur Koausscheidung von Übergangselementen in kristallinem Silizium. PhD thesis, Georg-August-Universität Göttingen, 2009.

[12] S. M. Myers, M. Seibt, and W. Schröter. Mechanisms of transition-metal gettering in silicon. Journal of Applied Physics, 88:3795-3819, 2000. 
[13] S. M. Joshi, U. M. Gösele, and T. Y. Tan. Improvement of minority carrier diffusion length in Si by Al gettering. Journal of Applied Physics, 77:3858-3863, 1995.

[14] P. S. Plekhanov, R. Gafiteanu, U. M. Gösele, and T. Y. Tan. Modeling of gettering of precipitated impurities from Si for carrier lifetime improvement in solar cell applications. Journal of Applied Physics, 86:2453-2458, 1999.

[15] R. Krain, S. Herlufsen, and J. Schmidt. Internal gettering of iron in multicrystalline silicon at low temperature. Applied Physics Letters, 93:152108, 2008.

[16] M. Seibt and V. Kveder. Gettering Processes and the Role of Extended Defects in Advanced Silicon Materials for Photovoltaic Applications. John Wiley \& Sons, Ltd, UK, 2012.

[17] D. Macdonald, A.Y. Liu, and S.P. Phang. External and internal gettering of interstitial iron in silicon for solar cells. Solid State Phenomena, 205-206:26-33, 2014.

[18] W. Seifert, G. Morgenstern, and M. Kittler. Influence of dislocation density on recombination at grain boundaries in multicrystalline silicon. Semiconductor Science and Technology, 8:1687, 1993.

[19] R. Rizk, X. Portier, G. Allais, and G. Nouet. Electrical and structural studies of copper and nickel precipitates in a $\Sigma=25$ silicon bicrystal. Journal of Applied Physics, 76:952-958, 1994.

[20] J. Bauer, O. Breitenstein, A. Lotnyk, and H. Blumtritt. Investigations on different types of filaments in multi-crystalline silicon for solar cells. The 22nd European Photovoltaic Solar Energy Conference Proceedings, 2007.

[21] O. Voß. Wechselwirkungen von Gold und Versetzungen in Silizium. PhD thesis, GeorgAugust-Universität Göttingen, 2009.

[22] P. Saring, C.Rudolf, L.Stolze, M. A. Falkenberg, and M.Seibt. Spatially resolved defect analysis in Cz-silicon after copper-nickel co-precipitation by virtue of light-beam induced current measurements. Solid State Phenomena, 431:156-158, 2010.

[23] P. Krenckel, P. Saring, M. A. Falkenberg, V. Kveder, and M. Seibt. Interaction of iron with extended defects in multicrystalline silicon studied by local gettering. Energy Procedia, 38:582 - 588, 2013.

[24] A.Y. Liu, Y. Fan, and D. Macdonald. Interstitial iron concentrations across multicrystalline silicon wafers via photoluminescence imaging. Progress in Photovoltaics: Research and Applications, 19:649-657, 2011.

[25] J. Schön, H. Habenicht, M. C. Schubert, and W. Warta. Understanding the distribution of iron in multicrystalline silicon after emitter formation: Theoretical model and experiments. Journal of Applied Physics, 109:063717, 2011. 
[26] T. Buonassisi, A. A. Istratov, M. A. Marcus, B. Lai, Z. Cai, S. M. Heald, and E.R. Weber. Engineering metal-impurity nanodefects for low-cost solar cells. Nature Materials, 4:676 $-679,2005$.

[27] J. Chen, D. Yang, Z. Xi, and T. Sekiguchi. Recombination activity of $\Sigma 3$ boundaries in boron-doped multicrystalline silicon: Influence of iron contamination. Journal of Applied Physics, 97:033701, 2005.

[28] P. S. Plekhanov, M. D. Negoita, and T. Y. Tan. Effect of Al-induced gettering and back surface field on the efficiency of Si solar cells. Journal of Applied Physics, 90:5388-5394, 2001.

[29] C. del Cañizo and A. Luque. A comprehensive model for the gettering of lifetime-killing impurities in silicon. Journal of The Electrochemical Society, 147:2685-2692, 2000.

[30] V. Kveder, W. Schröter, A. Sattler, and M. Seibt. Simulation of Al and phosphorus diffusion gettering in Si. Materials Science and Engineering B, 71:175-181, 2000.

[31] H. Hieslmair, S. Balasubramanian, A. Istratov, and E. R. Weber. Gettering simulator: physical basis and algorithm. Semiconductor Science and Technology, 16:567, 2001.

[32] A. Haarahiltunen, H. Väinölä, O. Anttila, E. Saarnilehto, M. Yli-Koski, J. Storgårds, and J. Sinkkonen. Modeling of heterogeneous precipitation of iron in silicon. Applied Physics Letters, 87:151908, 2005.

[33] M. Seibt, A. Sattler, C. Rudolf, O. Voß, V. Kveder, and W. Schröter. Gettering in silicon photovoltaics: current state and future perspectives. physica status solidi (a), 203, No. 4:696 - 713, 2006.

[34] J. Hofstetter, D. P. Fenning, M. I. Bertoni, J. F. Lelièvre, C. del Cañizo1, and T. Buonassisi. Impurity-to-efficiency simulator: predictive simulation of silicon solar cell performance based on iron content and distribution. Progress in Photovoltaics: Research and Applications, 19:487-497, 2011.

[35] A. Y. Liu, D. Walter, and D. Macdonald. Studying precipitation and dissolution of iron in multicrystalline silicon wafers during annealing. The 22nd International Photovoltaic Science and Engineering Conference Proceedings, 2012.

[36] A.A. Istratov, H. Hieslmair, and E.R. Weber. Iron and its complexes in silicon. Applied Physics A, 69:13-44, 1999.

[37] E. Olsen and E. J. Øvrelid. Silicon nitride coating and crucible-effects of using upgraded materials in the casting of multicrystalline silicon ingots. Progress in Photovoltaics: Research and Applications, 16:93-100, 2008.

[38] J. H. Reiss, R. R. King, and K. W. Mitchell. Characterization of diffusion length degradation in Czochralski silicon solar cells. Applied Physics Letters, 68:3302-3304, 1996. 
[39] D. Abdelbarey, V. Kveder, W. Schröter, and M. Seibt. Aluminum gettering of iron in silicon as a problem of the ternary phase diagram. Applied Physics Letters, 94:061912, 2009.

[40] D. Macdonald, J. Tan, and T. Trupke. Imaging interstitial iron concentrations in borondoped crystalline silicon using photoluminescence. Journal of Applied Physics, 103, 2008.

[41] S. Riepe, I. E. Reis, W. Kwapil, M. A. Falkenberg, J. Schön, H. Behnken, J. Bauer, D. Kreßner-Kiel, W. Seifert, and W. Koch. Research on efficiency limiting defects and defect engineering in silicon solar cells - results of the german research cluster solarfocus. physica status solidi (c), 8:733-738, 2011.

[42] P. Krenckel. Untersuchungen des Aluminiumgetterns metallischer Verunreinigungen in multikristallinem Silizium mittels ortsaufgelosten Photostrommessungen. Master's thesis, Georg-August-Universität Göttingen, 2012.

[43] M. A. Falkenberg, H. Schuhmann, M. Seibt, and V. Radisch. Localization and preparation of recombination-active extended defects for transmission electron microscopy analysis. Review of Scientific Instruments, 81(6):063705, 2010.

[44] M. Seibt, M. Griess, A. A. Istratov, H. Hedemann, A. Sattler, and W. Schröter. Formation and properties of copper silicide precipitates in silicon. physica status solidi (a), 166:171$182,1998$.

[45] A. A. Istratov and E. R. Weber. Physics of copper in silicon. Journal of The Electrochemical Society., 149, Issue 1:G21-G30, 2002.

[46] E. R. Weber. Transition metals in silicon. Applied Physics A, 30:1, 1983.

[47] T. Isobe, H. Nakashima, and K. Hashimoto. Diffusion coefficient of interstitial iron in silicon. Japanese Journal of Applied Physics, 28:1282, 1989.

[48] J. D. Murphy and R. J. Falster. Contamination of silicon by iron at temperatures below $800{ }^{\circ} \mathrm{C}$. physica status solidi (RRL), pages 1-3, 2011.

[49] Gottstein. Physikalische Grundlagen der Materialkunde. Springer-Verlag, Germany, 2nd edition, 2001.

[50] W. Schröter, V. Kveder, M. Seibt, A. Sattler, and E. Spiecker. Mechanisms and computer modelling of transition element gettering in silicon. Solar Energy Materials and Solar Cells, 72:299-313, 2002.

[51] W.Schröter and M.Seibt. Solubility and diffusion of transition metal impurities in c-Si. EMIS Datareview Series No 20. ”Properties of Crystalline Silicon”, 20:543-560, 1999.

[52] K. Graff. Metal Impurities in Silicon-Device Fabrication. Springers Series in Materials Science, Germany, 1999. 
[53] A.Y. Liu and D. Macdonald. Precipitation of interstitial iron in multicrystalline silicon. Solid State Phenomena, 205-206:34-39, 2014.

[54] M. Seibt, D. Abdelbarey, V. Kveder, C. Rudolf, P. Saring, L. Stolze, and O.Voß. Interaction of metal impurities with extended defects in crystalline silicon and its implications for gettering techniques used in photovoltaics. Materials Science and Engineering B, 159:264, 2009.

[55] S. P. Phang and D. Macdonald. Direct comparison of boron, phosphorus, and aluminum gettering of iron in crystalline silicon. Journal of Applied Physics, 109:073521, 2011.

[56] W. Schröter, M. Seibt, and D. Gilles. High-temperature properties of transition elements in silicon in: Handbook of Semiconductor Technology Vol. 1:Electronic Structure and Properties of Semiconductors. WILEY-VCH Verlag GmbH, 2000.

[57] M. Apel, I. Hanke, R. Schindler, and W. Schroeter. Aluminum gettering of cobalt in silicon. Journal of Applied Physics, 76:4432-4433, 1994.

[58] A. Sattler. Ratenlimitierende Prozesse beim Gettern von Kobalt in Silizium. PhD thesis, Georg-August-Universität Göttingen, 2003.

[59] S. Martinuzzi, I. Perichaud, and J. J. Simon. External gettering by aluminum-silicon alloying observed from carrier recombination at dislocations in float zone silicon wafers. Applied Physics Letters, 70:2744-2746, 1997.

[60] A. Luque, A. Moehlecke, R. Lagos, and C. Del Cañizo. Segregation model for Si gettering by Al. physica status solidi (a), 155:43-49, 1996.

[61] S. M. Joshi, U. M. Gösele, and T. Y. Tan. Extended high temperature Al gettering for improvement and homogenization of minority carrier diffusion lengths in multicrystalline Si. Solar Energy Materials and Solar Cells, 70:231 - 238, 2001.

[62] R. Kühnapfel and W. Schröter. in Semiconductor Silicon. Electrochemical Society, 1990.

[63] E.O. Sveinbjörnsson, O. Engström, and U. Sodervall. Phosphorus diffusion gettering of gold in silicon: The reversibility of the gettering process. Journal of Applied Physics, 73:7311, 1993.

[64] E. Spiecker, M. Seibt, and W. Schröter. Phosphorous-diffusion gettering in the presence of a nonequilibrium concentrationof silicon interstitials: A quantitative model. Physical Review B, 55:9577-9583, 1997.

[65] A. Voigt, E. Wolf, and H.P. Strunk. Grain orientation and grain boundaries in cast multicrystalline silicon. Materials Science and Engineering B, 54 Issue 3:202-206, 1998.

[66] J. L. Maurice and C. Colliex. Fast diffusers $\mathrm{Cu}$ and $\mathrm{Ni}$ as the origin of electrical activity in a silicon grain boundary. Applied Physics Letters, 55:241-243, 1989. 
[67] J. Chen, T. Sekiguchi, D. Yang, F. Yin, K. Kido, and S. Tsurekawa. Electron-beaminduced current study of grain boundaries in multicrystalline silicon. Journal of Applied Physics, 96:5490-5495, 2004.

[68] A. Zuschlag, H. Morhenn, J. Bernhard, J. Jung, S. Seren, and G. Hahn. Microscopic analysis of the influence of solar cell process steps on the recombination activity of extended crystal defects. The 25th European Photovoltaic Solar Energy Conference Proceedings, 2010.

[69] A.A. Istratov, T. Buonassis, W. Huber, and Weber E.R. Evidence for segregation of iron at grain boundaries in polycrystalline and multicrystalline silicon. Proceedings of the 14th NREL Workshop on Crystalline Silicon Solar Cell Materials and Processes, page 30-233, 2004.

[70] R.C. Dorward and J.S. Kirkaldy. Effect of grain-boundaries on the solubility of copper in silicon. Journal of Materials Science, 3:502-506, 1968.

[71] M. A. Falkenberg and M. Seibt. Transmission electron microscopy analysis of extended defects in multicrystalline silicon using in-situ EBIC/FIB sample preparation. physica status solidi (c), 10, No. 1:32-35, 2013.

[72] A. Ihlal and R. Rizk. Effects of iron contamination on the electrical activity of a silicon bicrystal. Journal of Physics D: Applied Physics, 29:3096-3100, 1996.

[73] X. Portier, A. Ihlal, and R. Rizk. Iron silicide formation by precipitation in a silicon bicrystal. physica status solidi (a), 161:75-84, 1997.

[74] B. Chen, J. Chen, T. Sekiguchi, M. Saito, and K. Kimoto. Structural characterization and iron detection at $\Sigma 3$ grain boundaries in multicrystalline silicon. Journal of Applied Physics, 105:113502, 2009.

[75] M. D. Pickett and T. Buonassisi. Iron point defect reduction in multicrystalline silicon solar cells. Applied Physics Letters, 92:122103, 2008.

[76] W. B. Henley and D. A. Ramappa. Iron precipitation in float zone grown silicon. Journal of Applied Physics, 82:589, 1997.

[77] A. Haarahiltunen, H. Väinölä, O. Anttila, M. Yli-Koski, and J. Sinkkonen. Experimental and theoretical study of heterogeneous iron precipitation in silicon. Journal of Applied Physics, 101:043507, 2007.

[78] M. Kittler, W. Seifert, M. Stemmer, and J. Palm. Interaction of iron with a grain boundary in borondoped multicrystalline silicon. Journal of Applied Physics, 77:3725, 1995.

[79] H. Habenicht, S. Riepe, O. Schultz, and W. Warta. Out-diffusion of metal from grain boundaries in multicrystalline silicon during thermal processing. The 22nd European Photovoltaic Solar Energy Conference Proceedings, 2007. 
[80] A.Y. Liu, D. Walter, S.P. Phang, and D. Macdonald. Investigating internal gettering of iron at grain boundaries in multicrystalline silicon via photoluminescence imaging. IEEE Journal of Photovoltaics, 2, No. 4:479, 2012.

[81] W. Shockley and W.T. Read. Statistics of the recombinations of holes and electrons. Physical Review, 87:835, 1952.

[82] P. Blood and J.W. Orton. The electrical characterization of seminconductors: majority carriers and electron states. Academic Press Inc., USA, 1992.

[83] L. J. Geerligs and D. Macdonald. Dynamics of light-induced dissociation of FeB pairs in crystalline silicon. Applied Physics Letters, 85:5227, 2004.

[84] C. Donolato. Theory of beam induced current characterization of grain boundaries in polycrystalline solar cells. Journal of Applied Physics, 3:54, 1983.

[85] S.M. Sze and K.K. Ng. Physics of Semiconductor Devices. John Wiley \& Sons, Inc., USA, 2006.

[86] S. Riepe, I.E. Reis, and W. Koch. Solar silicon material research network solarfocus (solarsilizium forschungscluster). The 23rd European Photovoltaic Solar Energy Conference Proceedings, 2008.

[87] S. Riepe, I. E. Reis, W. Kwapil, M. A. Falkenberg, J. Schön, H. Behnken, J. Bauer, D. Kreßner-Kiel, W. Seifert, and W. Koch. Research on efficiency limiting defects and defect engineering in silicon solar cells - results of the german research cluster solarfocus. physica status solidi (c) 8, 3:733 - 738, 2011.

[88] E. Nes and J. Washburn. Precipitate colonies in silicon. Journal of Applied Physics, 43:4, 2005-2006 (1972).

[89] Michael Seibt, Reda Khalil, Vitaly Kveder, and Wolfgang Schröter. Electronic states at dislocations and metal silicide precipitates in crystalline silicon and their role in solar cell materials. Applied Physics A, 96:235-253, 2009.

[90] M. Trushin, Steven M. Heald, Z. C. W. Seifert, O. Vyvenko, J. Bauer, G. MartinezCriadoand M. Salome, and M. Kittler. XBIC/ $\mu$-XRF / $\mu$-XAS analysis of metals precipitation in block-cast solar silicon. Nuclear Instruments and Methods in Physics Research $B, 268: 254-258,2010$.

[91] J. Nitzbon. Entwicklung eines LBIC-Modells zur Simulation der defektverursachten Rekombinationsaktivität mit der Finite-Elemente-Methode, Bachelor's thesis, GeorgAugust-Universität Göttingen, 2013.

[92] T. Y. Tan, R. Grafiteanu, S. M. Joshi, and U. Gösele. Science and Modelling of Impurity Gettering in Silicon in Semiconductor Silicon, volume PV-98-1. The Electrochemical Society, Pennington, NJ, 1998. 
[93] D.A. Antoniadis and R.W. Dutton. Models for computer simulation of complete ic fabrication process. IEEE Transactions on Electron Devices, 26:490-500, 1979.

[94] R. W. Balluffi, S. M. Allen, and W. C. Carter. Kinetics of Materials. John Wiley \& Sons Inc., USA, 2005.

[95] C.P. Flynn. Point Defects and Diffusion. Clarendon Press, England, 1972.

[96] F. S. Ham. Theory of diffusion-limited precipitation. Journal of Physics and Chemistry of Solids, 6:335-351, 1958.

[97] P. Käshammer and T. Sinno. Interactions of twin boundaries with intrinsic point defects and carbon in silicon. Journal of Applied Physics, 114:083505, 2013.

[98] Y. Du, Y.A. Chang, B. Huang, W. Gong, H. Xu Z. Jin, Z. Yuan, Y. Liu, Y. He, and F.-Y. Xie. Diffusion coefficients of some solutes in fcc and liquid $\mathrm{Al}$ : critical evaluation and correlation. Materials Science and Engineering A, 363:140-151, 2003.

[99] D. Macdonald, A. Cuevas, and J. Wong-Leung. Capture cross sections of the acceptor level of iron-boron pairs in p-type silicon by injection-level dependent lifetime measurements. Journal of Applied Physics, 89:7932-7939, 2001.

[100] D. P. Fenning, J. Hofstetter, M. I. Bertoni, S. Hudelson, and M. Rinio et al. Iron distribution in silicon after solar cell processing: Synchrotron analysis and predictive modeling. Applied Physics Letters, 98:162103, 2011.

[101] H. Behnken. Personal communication. Access, Achen, 2009.

[102] A.Y. Liu, D. Walter, S. P. Phang, and D. Macdonald. Imaging and modelling the internal gettering of interstitial iron by grain boundaries in multicrystalline silicon. IEEE Journal of Photovoltaics, 2011.

[103] AnYao Liu. Private communication. October 2013.

[104] M.C. Schubert, H. Habenicht, and W. Warta. Imaging of metastable defects in silicon. IEEE Journal of Photovoltaics 1, 2:168-173, 2011.

[105] D. Macdonald, T. Roth, P. N. K. Deenapanray, T. Trupke, and R. A. Bardos. Doping dependence of the carrier lifetime crossover point upon dissociation of iron- boron pairs in crystalline silicon. Applied Physics Letters, 89:142107, 2006.

[106] COMSOL. COMSOL Multiphysics User's Guide. COMSOL, 1998-2011. 


\section{Acknowledgement}

This work was partly supported by the German Federal Ministry for the Environment, Nature Conservation and Nuclear Safety via research clusters SolarFocus (0327650 B) and SolarWinS $(0327259 \mathrm{~B})$ projects and the industry partners. The content of this publication is the responsibility of the author.

First and foremost I want to thank my advisor, professor Michael Seibt, for enabling this thesis. I appreciate that he was always available for discussions and contributed to my work by advice and ideas. I am especially thankful for the family friendly conditions he made always possible. I am grateful to my co-advisor, professor Christian Jooss, for his interest on my work.

I thank professor Vitaly Kveder from the Institute of Solid State Physics RAS, Chernogolovka for being a source of advice to me concerning the physical model setup, the relevant literature and numerical problems.

I would like to acknowledge all members of the Seibt group for the pleasant environment and valuable discussions. My special thanks belongs to Patricia Krenckel for supplying me with LBIC data for comparison with my simulations and for exchanging ideas. I would like to thank Jan Nitzbon for the development of the LBIC model, Henning Schumann for helping me out at the FIB and TEM, Philipp Saring for discussions and setting up the EBIC with me and Bettina Schlieper-Ludewig for introducing me to the lab and helping me with chemical sample preparation.

I would like to thank Volker Radisch from the lab group of the Institute of Material Physics, who is always open to share his knowledge and his tricks on FIB sample preparation with anyone. I am grateful to Thomas Lehmann from the fine mechanics and electronics group for building the EBIC sample holder for us.

I especially appreciate the advice from Dr. Kodanda Ram Mangipudi from the Institute of Material Physics on modeling with comsol and the guidance on FEM from professor Marc Avila from the University of Erlangen.

My special thanks belongs to AnYao Liu from the Australian National University, for willingly sharing her PL data and her ideas with me.

I would like to thank my family and friends for all their love, encouragement and support. Especially I want to thank John Tuepker for reviewing my English. I thank my parents Jutta and Peter Tyra for supporting me during studying physics and throughout my life. I am grateful to them and my parents-in-law Erika and Norbert Falkenberg for their loving care for Solveigh whenever it is needed. I am thankful to my loving husband Hilmar Falkenberg for his support and encouragement. With his patience and serenity he contributed a lot to this work. I thank my daughter Solveigh for always reminding me that there is a life beyond physics. 


\section{Lebenslauf:}

Name: $\quad$ Marie Aylin Falkenberg, geborene Tyra

Geburtsdatum und -ort: 17. November 1982 in Göttingen

\section{Beruflicher Werdegang}

$1989-1995$

Grund- und Orientierungsschule Göttingen

$1995-2002$

Max-Planck-Gymnasium Göttingen

Okt. 2002 - Sep. 2004 Physikstudium an der Christian-Albrechts-Universität zu Kiel

Okt. 2004 - Dez. 2007 Physikstudium an der Georg-August-Universität Göttingen

Diplomarbeit im Institut für Astrophysik: „Galaxy Transformation in Galaxy Clusters: The Role of $\mathrm{E}+\mathrm{A}$ and Post-Starburst Galaxies"

Mrz. 2008 - Sep. 2008 Praktikum am Laser-Laboratorium Göttingen e.V. (LLG)

Sep. 2008 - Sep.2014 Promotionsstudium und Tätigkeit als wissenschaftliche Mitarbeiterin am IV. Physikalischen Institut der Georg-AugustUniversität Göttingen

Thema der Dissertation: „Metal Impurity Redistribution in Crystalline Silicon for Photovoltaic Application“ 